Existência e multiplicidade de soluções para uma classe de problemas quasilineares com crescimento crítico exponencial

\author{
Luciana Roze de Freitas
}




\title{
Existência e multiplicidade de soluções para uma classe de problemas quasilineares com crescimento crítico exponencial
}

\author{
Luciana Roze de Freitas \\ Orientador: Prof. Dr. Claudianor Oliveira Alves \\ Co-orientador: Prof. Dr. Sérgio Henrique Monari Soares \\ Tese apresentada ao Instituto de Ciências Matemáticas e de \\ Computação - ICMC-USP, como parte dos requisitos para \\ obtenção do título de Doutor em Ciências - Matemática.
}


Aos meus pais Lourenço (in memoriam) e Geraldina, com amor e gratidão. 
Ao meu orientador, Prof. Claudianor O. Alves, com quem tive a honra de trabalhar, sou grata por todos os ensinamentos e por dedicar-se a este projeto com tão notável profissionalismo e inteligência. Agradeço também pela oportunidade, pelo incentivo e por despertar ainda mais o meu interesse pela pesquisa.

Aos professores do ICMC-USP pela excelência no ensino. Em especial, ao meu coorientador, Prof. Sérgio H. M. Soares, por todo o apoio e atenção.

Aos professores Marcelo Fernandes Furtado, Marcelo da Silva Montenegro e Francisco Odair Vieira de Paiva por aceitarem participar da comissão julgadora e pelas valiosas sugestões apresentadas.

A UEPB pelo estímulo à capacitação docente, especialmente aos professores do Departamento de Matemática.

Aos professores do DME-UFCG pela contribuição dada à minha formação acadêmica.

Ao meu marido e meu filho por todo o amor e companheirismo.

Aos meus pais pela educação que me proporcionaram e pela enorme ajuda que recebi, sem a qual não conseguiria seguir com os estudos. Agradeço também as minhas irmãs e irmão pelo afeto e pela incansável torcida, especialmente à Silvia, a quem agradeço profundamente por toda a assistência.

Não poderia deixar de agradecer as queridas amigas e colegas de profissão Claudilene e Isabelle pela amizade e incentivo.

Finalmente, agradeço a todos que de alguma forma contribuíram para a realização deste trabalho. 
\begin{tabular}{|l|}
\hline RESUMO \\
RESO
\end{tabular}

Neste trabalho, mostramos a existência e multiplicidade de soluções para a seguinte classe de equações elípticas quasilineares

$$
\left\{\begin{array}{l}
-\Delta_{N} u+|u|^{N-2} u=f(x, u), \quad x \in \Omega, \\
u \neq 0, u \in W^{1, N}(\Omega),
\end{array}\right.
$$

onde $\Omega$ é um domínio em $\mathbb{R}^{N}, N \geq 2, \Delta_{N}$ é o operador $N$-Laplaciano e $f$ é uma função que possui um crescimento crítico exponencial. Para obter nossos resultados utilizamos o Princípio Variacional de Ekeland, Teorema do Passo da Montanha, Categoria de LusternikSchnirelman, Ação de Grupo e técnicas baseadas na Teoria do Gênero.

Palavras chaves: Problemas elípticos quasilineares, Método Variacional, N-Laplaciano, crescimento crítico exponencial, Princípio Variacional de Ekeland, Categoria de LusternikSchnirelman, Desigualdade de Trudinger-Moser. 
In this work, we show the existence and multiplicity of solutions for the following class of quasilinear elliptic equations

$$
\left\{\begin{array}{l}
-\Delta_{N} u+|u|^{N-2} u=f(x, u), \quad x \in \Omega, \\
u \neq 0, u \in W^{1, N}(\Omega)
\end{array}\right.
$$

where $\Omega$ is a domain in $\mathbb{R}^{N}, N \geq 2, \Delta_{N}$ is the N-Laplacian operator and $f$ is a function with exponential critical growth. To obtain our results we utilize the Ekeland Variational Principle, the Mountain Pass Theorem, Lusternik-Schnirelman of Category, Group Action and techniques based on Genus Theory.

Key words: Quasilinear elliptic problems, Variational Methods, N-Laplacian, exponential critical growth, Ekeland Variational Principle, Lusternik-Schnirelman of Category, Trudinger-Moser inequality. 
Neste texto usaremos as seguintes notações:

$\mathbb{R}^{N}: \quad \quad \quad \quad$ Espaço Euclidiano N-dimensional com a norma $|x|=\left(\sum_{i=1}^{N} x_{i}^{2}\right)^{\frac{1}{2}}, x \in \mathbb{R}^{N}$.

$\partial D, \bar{D}, D^{c}:$ Fronteira, fecho e complementar do conjunto $D$, respectivamente.

$|D|: \quad$ Medida de Lebesgue do conjunto $D$.

$B_{r}(x)$ Bola aberta de centro $x$ e raio $r$

$\nabla u: \quad \quad$ Gradiente da função $u$, isto é, $\nabla u=\left(\frac{\partial u}{\partial x_{1}}, \frac{\partial u}{\partial x_{2}}, \ldots, \frac{\partial u}{\partial x_{N}}\right)$.

$\operatorname{Dom}(h)$ : Domínio da função $h$.

Suppu: $\quad$ Suporte da função $u$ (ver [32], pág. 68).

q.t.p.: $\quad$ Quase todo ponto, ou seja, a menos de um conjunto de medida nula.

$C(\Omega): \quad$ Espaço das funções contínuas definidas em $\Omega$.

$C^{k}(\Omega): \quad$ Espaço das funções definidas em $\Omega$ que possuem todas as derivadas de ordem menor ou igual a $k$ contínuas.

$C_{0}^{\infty}(\Omega): \quad$ Conjunto de todas as funções $u \in C^{\infty}(\Omega)$ tal que $\operatorname{supp}(u) \subset \subset \Omega$.

$L^{p}(\Omega): \quad$ Espaço das funções mensuráveis $u: \Omega \rightarrow \mathbb{R}$ tais que $\int_{\Omega}|u|^{p} d x<\infty, 1 \leq p<\infty$.

$L^{\infty}(\Omega): \quad$ Espaço das funções mensuráveis $u: \Omega \rightarrow \mathbb{R}$ tais que existe $C>0$ com $|u(x)| \leq C$ q.t.p. em $\Omega$. 
$L_{\text {loc }}^{p}(\Omega): \quad \quad$ Espaço das funções mensuráveis $u: \Omega \rightarrow \mathbb{R}$ tais que $\int_{K}|u|^{p} d x<\infty$, para todo conjunto compacto $K \subset \Omega, 1 \leq p<\infty$.

$W^{1, N}(\Omega)$ : $\quad$ Espaço de Sobolev das funções em $L^{N}(\Omega)$ que possuem as derivadas fracas de primeira ordem pertencentes a $L^{N}(\Omega)$.

$W_{0}^{1, N}(\Omega): \quad$ Fecho de $C_{0}^{\infty}(\Omega)$ em $W^{1, N}(\Omega)$.

$H^{\prime}:$

Dual topológico do espaço vetorial normado $H$.

$|u|_{p}$

Norma em $L^{p}$ dada por $|u|_{p}=\left(\int_{\Omega}|u|^{p} d x\right)^{\frac{1}{p}}$.

$|u|_{\infty}:$

Norma em $L^{\infty}(\Omega)$, ou seja, $|u|_{\infty}=\inf \{C ;|u(x)| \leq C$ q.t.p. em $\Omega\}$.

$\|\cdot\|:$

Norma nos espaços $W^{1, N}(\Omega)$ ou $W_{0}^{1, N}(\Omega)$.

$X \hookrightarrow Y:$

Imersão contínua de $X$ em $Y$, isto é, $X \subset Y$ e a inclusão $i: X \rightarrow Y$

é uma aplicação contínua.

$X \underset{\operatorname{comp}}{\hookrightarrow} Y:$

Imersão compacta de $X$ em $Y$, isto é, $X \subset Y$ e a inclusão $i: X \rightarrow Y$

é um operador compacto.

$\rightarrow, \rightarrow$ :

Convergência forte e convergência fraca, respectivamente.

$o_{n}(1):$

Sequência de números reais convergindo para 0 quando $n \rightarrow+\infty$.

$\operatorname{cat}_{X}(A): \quad$ Categoria do conjunto $A \subset X$ no espaço topológico $X$.

$\gamma(A): \quad$ Gênero do conjunto $A$.

$u_{+}, u^{+}: \quad$ Parte positiva da função $u$, isto é, $u^{+}=u_{+}=\max \{u, 0\}$.

$u_{-}, u^{-}: \quad$ Parte negativa da função $u$, isto é, $u^{-}=u_{-}=\max \{-u, 0\}$.

$C_{1}, C_{2}, C_{3}: \quad$ Constantes reais possivelmente diferentes.

$C_{\beta}, C(\beta): \quad$ Constante real que depende do valor $\beta$.

$(P . S)_{c}: \quad$ Sequência Palais-Smale no nível $c$.

$g=o(h), s \rightarrow 0: \quad$ Significa que $\lim _{s \rightarrow 0} \frac{g(s)}{h(s)}=0$. 
Introdução

1 Existência de solução para um problema indefinido em domínio exterior 15

1.1 Preliminares . . . . . . . . . . . . . . . . . . . . . . 17

1.2 Propriedades da sequência Palais-Smale . . . . . . . . . . . . . . . . . . . . . 26

1.3 Existência de solução . . . . . . . . . . . . . . . . . . . . . . . . . . . . . . 34

2 Multiplicidade de soluções via minimização local e Passo da Montanha 37

2.1 Soluções para uma classe de equações em domínio limitado . . . . . . . . . 37

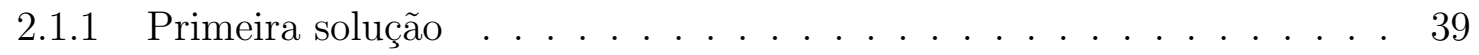

2.1 .2 Segunda solução . . . . . . . . . . . . . . . . . . . . . . 46

2.1 .3 Multiplicidade de soluções com energia negativa . . . . . . . . . . . . 49

$2.2 \quad$ Soluções para uma classe de equações em $\mathbb{R}^{N} \ldots \ldots \ldots$. . . . . . . . . . . . . . 53

2.2 .1 Primeira solução . . . . . . . . . . . . . . . . . . 55

2.2 .2 Segunda solução . . . . . . . . . . . . . . . . . . . 65

2.2 .3 Multiplicidade de soluções com energia negativa $\ldots$. . . . . . . . . . . 67

3 Multiplicidade de soluções via Teoria do Gênero e um problema com $\begin{array}{ll}\text { quebra de simetria } & 69\end{array}$

3.1 Soluções para uma classe de equações elípticas em domínio limitado . . . . . 69

3.1 .1 Teorema abstrato . . . . . . . . . . . . . . . . . . . 72

3.1 .2 Multiplicidade de soluções $\ldots$. . . . . . . . . . . . . . . . . . . . 77

3.2 Multiplicidade de soluções não radiais: Quebra de Simetria . . . . . . . . . . 83

3.2 .1 Preliminares . . . . . . . . . . . . . . . . . . . . . . . . . . . 84 
$3.2 .2 \quad$ Propriedades dos níveis $J_{k, r} \ldots \ldots \ldots \ldots$. . . . . . . . . . . 87

3.2 .3 Um resultado de multiplicidade . . . . . . . . . . . . . . . . . . . 96

\begin{tabular}{|lll|}
\hline & Multiplicidade de soluções via categoria de Lusternik-Schnirelman & 97
\end{tabular}

4.1 Preliminares . . . . . . . . . . . . . . . . . . . . . . . . . . . . . . . . . . . . . . . . . . . . . . . 99

4.2 Um resultado de compacidade . . . . . . . . . . . . . . . . . . 100

4.3 Comportamento dos níveis minimax . . . . . . . . . . . . . . . 106

$4.4 \quad$ Um resultado de multiplicidade . . . . . . . . . . . . . . . . . . . . . . . . 113

\begin{tabular}{ll}
\hline A Resultados Gerais & 117
\end{tabular}

A.1 Desigualdades . . . . . . . . . . . . . . . . . . . . . . . . . . 117

A.2 Resultados de convergência . . . . . . . . . . . . . . . . . . . . . . 118

A.3 Resultados de imersão $\ldots \ldots \ldots$. . . . . . . . . . . . . . . . . . . . 119

A.4 Teorema de Deformação . . . . . . . . . . . . . . . . . . . . . 121

A.5 Teorema do Passo da Montanha . . . . . . . . . . . . . . . . . . . . . . 122

A.6 Princípio Variacional de Ekeland . . . . . . . . . . . . . . . . . . . . . . . . 123

A.7 Princípio da Criticalidade Simétrica de Palais . . . . . . . . . . . . . . . . . 124

A.8 Gênero . . . . . . . . . . . . . . . . . . . . . . . . . . . . . . . . . 124

A.9 Categoria . . . . . . . . . . . . . . . . . . . . . . 125

\begin{tabular}{ll}
\hline Referências Bibliográficas & 127
\end{tabular} 
A proposta deste trabalho é estudar resultados de existência e multiplicidade de soluções para a seguinte classe de problemas elípticos quasilineares

$$
\left\{\begin{array}{l}
-\Delta_{N} u+|u|^{N-2} u=f(x, u), \quad x \in \Omega \\
u \neq 0, u \in W_{0}^{1, N}(\Omega)
\end{array}\right.
$$

onde $\Omega \subset \mathbb{R}^{N}$ é um domínio não necessáriamente limitado, $N \geq 2$ e $\Delta_{N}$ denota o operador $N$-Laplaciano

$$
\Delta_{N} u=\operatorname{div}\left(|\nabla u|^{N-2} \nabla u\right)=\sum_{i=1}^{N} \frac{\partial}{\partial x_{i}}\left(|\nabla u|^{N-2} \frac{\partial u}{\partial x_{i}}\right)
$$

Iremos analisar alguns problemas variantes de $(P)$, onde em cada um deles será assumido um conjunto específico de hipóteses sobre $f$ e $\Omega$. Nos casos em que o domínio $\Omega$ possui fronteira, consideramos a condição de Dirichlet na fronteira.

Em diversas áreas da Matemática Aplicada, Física e Mecânica podemos encontrar fenômenos que podem ser modelados com equações envolvendo o operador $p$-Laplaciano, $p>1$. Tal operador surge, por exemplo, no estudo de Fluidos não Newtonianos, na teoria da elasticidade não linear, em problemas de reação e difusão, no estudo de geleiras (Glaciologia), extração de petróleo e etc. Para mais detalhes sobre estas e outras aplicações do operador $p$-Laplaciano citamos os artigos [30], [50], [56], [15], 64], 69] e suas referências. 
Uma solução para o problema $(P)$ com condição de Dirichlet é, por definição, uma função $u \in W_{0}^{1, N}(\Omega) \backslash\{0\}$ que satisfaz

$$
\int_{\Omega}\left(|\nabla u|^{N-2} \nabla u \nabla v+|u|^{N-2} u v\right) d x=\int_{\Omega} f(x, u) v d x, \quad \forall v \in W_{0}^{1, N}(\Omega) .
$$

Se $\Omega=\mathbb{R}^{N}$, as soluções de $(P)$ são funções $u \in W^{1, N}\left(\mathbb{R}^{N}\right) \backslash\{0\}$ que verificam 0.1 no referido espaço.

Em nosso estudo, utilizamos métodos variacionais, explorando diferentes técnicas para obtenção de pontos críticos do funcional energia $I$ associado a $(P)$, que é definido por

$$
I(u)=\frac{1}{N} \int_{\Omega}\left(|\nabla u|^{N}+|u|^{N}\right) d x-\int_{\Omega} F(x, u) d x,
$$

onde $F(x, s)=\int_{0}^{s} f(x, t) d t$. Os pontos críticos do funcional $I$ satisfazem (0.1) (ver Observação 1.2, portanto, são soluções para o problema $(P)$.

Dizemos que uma função $g$ possui crescimento crítico exponencial no infinito (veja [2, 44, 26]) se satisfaz a seguinte condição:

$\left(\mathbf{H}_{\mathbf{c}}\right)$ existe $\alpha_{0}>0$ tal que

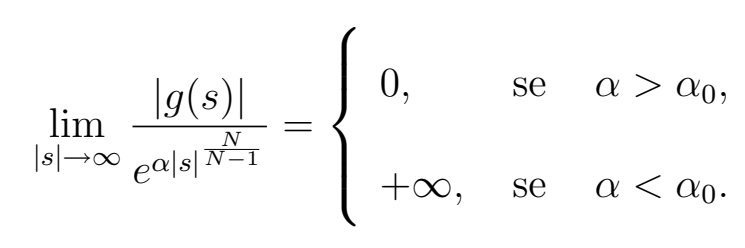

Em todos os problemas abordados, iremos trabalhar com não linearidades que possuem crescimento crítico exponencial. Quando $\Omega$ é um domínio limitado, a condição $\left(H_{c}\right)$ é motivada pelas seguintes estimativas provadas por Trudinger [77] e Moser [60]:

$$
e^{\alpha|u|^{\frac{N}{N-1}}} \in L^{1}(\Omega), \quad \forall u \in W_{0}^{1, N}(\Omega), \quad \forall \alpha>0
$$

e

$$
\sup _{\|u\| \|_{W_{0}^{1, N}} \leq 1} \int_{\Omega} e^{\alpha|u|^{\frac{N}{N-1}}} d x \leq C(N,|\Omega|) \in \mathbb{R}, \quad \forall \alpha \leq \alpha_{N}=N \omega_{N-1}^{\frac{1}{N-1}}
$$

onde $\omega_{N-1}$ é a medida $(N-1)$-dimensional da $(N-1)$-esfera. Versões destas desigualdades para domínios ilimitados foram provadas por Cao [35], para $N=2$ e por Bezerra do Ó [27], para o caso $N \geq 2$. Tais versões serão fortemente utilizadas ao longo deste trabalho, a fim de deduzir as principais estimativas. 
No Capítulo 1, estudamos o problema $(P) \operatorname{com} \Omega$ sendo um domínio exterior da forma $\Omega=\mathbb{R}^{N} \backslash \Theta, N \geq 2$, onde $\Theta \subset \mathbb{R}^{N}$ é um domínio limitado com fronteira suave. Além disso, vamos considerar

$$
f(x, u):=a(x) g(u),
$$

onde as funções $a$ e $g$ verificam algumas hipótese que listaremos abaixo. Nosso objetivo é provar a existência de solução não trivial para o problema

$$
\left\{\begin{array}{l}
-\Delta_{N} u+|u|^{N-2} u=a(x) g(u), \quad x \in \Omega, \\
u=0, \quad x \in \partial \Omega .
\end{array}\right.
$$

Vamos supor que a função $a(x)$ satisfaz as seguintes hipóteses:

$\left(\mathbf{a}_{1}\right) a: \Omega \rightarrow \mathbb{R}$ é uma função contínua que muda de sinal em $\Omega$;

$\left(\mathbf{a}_{2}\right) \operatorname{dist}\left(\overline{\Omega^{+}}, \overline{\Omega^{-}}\right)=\delta>0$, onde $\Omega^{+}=\{x \in \Omega ; a(x)>0\}, \quad \Omega^{-}=\{x \in \Omega ; a(x)<0\} \mathrm{e}$ dist é a função distância entre conjuntos;

(a) $a \in L^{\infty}\left(\Omega^{-}\right)$e existe $R>0$ tal que $a(x)<0$, para $|x| \geq R$.

Para a função $g$, além da condição de crescimento crítico exponencial $\left(H_{c}\right)$, admitimos o seguinte conjunto de hipóteses:

$\left(\mathbf{H}_{\mathbf{0}}\right) g: \mathbb{R} \rightarrow \mathbb{R}$ é uma função contínua tal que

$$
g(s)=o\left(|s|^{N-1}\right), \text { quando }|s| \rightarrow 0
$$

$\left(\mathbf{H}_{\mathbf{1}}\right)$ existem $\nu>0$ e $0<\theta<\frac{\nu}{N+(N-1) M}$ tais que

$$
0<\frac{\nu}{\theta} G(s) \leq g(s) s, \quad \forall|s|>0,
$$

onde $G(s)=\int_{0}^{s} g(t) d t$ e $M:=\sup _{\Omega}|\nabla \zeta|<\infty$, com $\zeta \in C^{\infty}(\Omega)$ sendo uma função fixada verificando

$$
0 \leq \zeta \leq 1, \quad \zeta(x)=1, x \in \Omega^{+} \quad \text { e } \quad \zeta(x)=0, x \in \Omega^{-}
$$

$\left(\mathbf{H}_{\mathbf{2}}\right)$ existem constantes $K_{0}, R_{0}>0$ tais que

$$
0<G(s) \leq K_{0} g(s), \quad \forall|s| \geq R_{0} ;
$$

$\left(\mathbf{H}_{\mathbf{3}}\right) \lim _{|s| \rightarrow \infty} s g(s) e^{-\alpha_{0}|s|^{\frac{N}{N-1}}}=+\infty$. 
Hipóteses similares a $\left(H_{2}\right)$ e $\left(H_{3}\right)$ são consideradas, por exemplo, nos artigos Adimurthi [2], de Figueiredo, Miyagaki \& Ruf [44] e Bezerra do Ó [27], onde também podemos encontrar resultados de existência de solução para problemas elípticos envolvendo crescimento exponencial.

O problema $\left(P_{1}\right)$ tem sua origem na seguinte equação

$$
-\Delta u=a(x) g(u), \quad x \in \Omega
$$

onde $a: \Omega \rightarrow \mathbb{R}$ é uma função que muda de sinal em $\Omega$. Uma tal condição sobre $a$ atribui um carácter indefinido ao problema. Na literatura, é possível encontrar diversos trabalhos que tratam questões de existência e multiplicidade para problemas indefinidos. Para domínios limitados podemos citar Alama \& Tarantello [4, 5], Berestycki, CapuzzoDolcetta \& Nirenberg [22, 23], Tehrani [75] e as referências neles contidas. Em particular, em [4] os autores consideraram o problema

$$
\left\{\begin{array}{l}
-\Delta u-\lambda u=a(x) g(u), \quad x \in \Omega \subset \mathbb{R}^{N}(N \geq 3), \\
u=0, \quad x \in \partial \Omega
\end{array}\right.
$$

onde $a \in C(\bar{\Omega})$ muda de sinal e $g$ possui crescimento polinomial subcrítico. Questões interessantes que relacionam a existência de soluções positivas e o termo $a(x)$ foram observadas, por exemplo, em [4, 22] os autores provaram que a condição $\int a(x) \phi_{1}^{q} d x<0$ é necessária e suficiente para a existência de solução positiva quando $g(u)=|u|^{q-2} u$, $2<q<2^{*}$, onde $\phi_{1}$ é a primeira autofunção positiva para $-\Delta$ em $W_{0}^{1,2}(\Omega)$ e $2^{*}=\frac{2 N}{N-2}$ é o expoente crítico de Sobolev.

Costa \& Tehrani [41] estenderam em parte, para o espaço $\mathbb{R}^{N}$, os resultados obtidos em [4], analisando a existência e multiplicidade de soluções positivas para o problema

$$
-\Delta u-\lambda h(x) u=a(x) g(u), \quad u>0 \quad \text { em } \mathbb{R}^{N}(N \geq 3),
$$

onde $0 \leq h(x) \in L^{\frac{N}{2}}\left(\mathbb{R}^{N}\right) \cap L^{\alpha}\left(\mathbb{R}^{N}\right), \alpha>\frac{N}{2}$ e a é uma função que muda de sinal e satisfaz $\lim _{|x| \rightarrow \infty} a(x)=a_{\infty}<0$. Novamente, a necessidade da condição $\int a(x) \phi_{1}^{q} d x<0$ foi verificada.

Ainda no caso subcrítico, Tehrani [74] considerou o problema 0.2 com $\Omega$ sendo domínio exterior em $\mathbb{R}^{N}, N \geq 3$. Admitindo hipóteses similares a $\left(a_{1}\right)-\left(a_{3}\right)$, foram provados resultados de existência de solução positiva e multiplicidade para o caso em que g é ímpar. 
Para equações elípticas envolvendo crescimento crítico citamos o artigo de Morais Filho \& Miyagaki [58], onde os autores estabeleceram resultados de existência para o problema envolvendo expoente crítico de Sobolev

$$
-\Delta u=a(x) g(u)+u|u|^{2^{*}-2}, \quad x \in \mathbb{R}^{N}(N \geq 3),
$$

onde $a$ é uma função descontínua que muda de sinal e $g$ possui crescimento subcrítico.

Com a análise do problema $\left(P_{1}\right)$ complementamos o estudo feito em [74] no sentido de que estamos considerando o caso em que o termo não linear admite um crescimento crítico exponencial e também propondo o estudo para o operador $N$-Laplaciano. Considerar a não linearidade com crescimento crítico impõe alguns cuidados no tratamento variacional, pois, neste caso, o funcional energia não irá satisfazer a condição Palais-Smale. Lembramos também que o $N$-Laplaciano é um operador não linear que, comparado ao operador Laplaciano, requer mais empenho na justificativa de algumas estimativas.

O principal resultado do Capítulo 1 é o seguinte:

Teorema 1 Suponha que a função a satisfaz as hipóteses $\left(a_{1}\right)-\left(a_{3}\right)$ e g satisfaz $\left(H_{c}\right)$, $\left(H_{0}\right),\left(H_{1}\right),\left(H_{2}\right)$ e $\left(H_{3}\right)$. Então, o problema $\left(P_{1}\right)$ possui pelo menos uma solução.

Para provar o Teorema 1 nossos principais argumentos são baseados na Desigualdade de Trudinger-Moser e no Teorema do Passo da Montanha sem a condição Palais-Smale.

No Capítulo 2, abordamos dois problemas nos quais o termo não linear consiste de uma perturbação do termo crítico. Reservamos a primeira seção para estudar o seguinte problema

$$
\left\{\begin{array}{l}
-\Delta_{N} u=\lambda|u|^{q-2} u+g(u), \quad x \in \Omega, \\
u=0, \quad x \in \partial \Omega
\end{array}\right.
$$

onde $\Omega \subset \mathbb{R}^{N}$ é um domínio limitado com fronteira suave, $N \geq 2,1<q<N$ e $\lambda>0$.

Na segunda seção, estudamos o problema

$$
\left\{\begin{array}{l}
-\Delta_{N} u+|u|^{N-2} u=\lambda h(x)|u|^{q-2} u+g(u), \quad x \in \mathbb{R}^{N}, \\
u \in W^{1, N}\left(\mathbb{R}^{N}\right),
\end{array}\right.
$$

onde $1<q<N$, $h$ é uma função positiva que pertence a $L^{\frac{N}{N-q}}\left(\mathbb{R}^{N}\right)$ e $\lambda>0$. 
Para a função $g$ admitimos as hipóteses $\left(H_{c}\right),\left(H_{0}\right)$ e as seguintes hipóteses que também serão comuns aos demais capítulos:

$\left(\mathbf{H}_{4}\right)$ existe $\nu>N$ tal que

$$
0<\nu G(s) \leq g(s) s, \quad \forall|s|>0
$$

onde $G(s)=\int_{0}^{s} g(t) d t$

$\left(\mathbf{H}_{\mathbf{5}}\right)$ existem $p>N$ e uma constante $C_{p}>0$ tais que

$$
g(s) \geq C_{p} s^{p-1}, \quad \forall s \geq 0
$$

Apenas para o estudo do problema $\left(P_{3}\right)$ adicionamos as hipóteses:

$\left(\mathbf{H}_{6}\right) g \in C^{1}(\mathbb{R}, \mathbb{R})$ é tal que $\frac{g(s)}{s^{N-1}}$ é crescente em $(0,+\infty) ;$

$\left(\mathbf{H}_{\mathbf{7}}\right)$ existe $C>0$ tal que

$$
\left|g^{\prime}(s)\right| \leq C e^{\alpha_{0}|s|^{\frac{N}{N-1}}}, \forall s \in \mathbb{R}
$$

É importante observar que a constante $C_{p}$ da hipótese $\left(H_{5}\right)$ será, em alguns problemas, determinada adequadamente. Tal hipótese foi inicialmente considerada em Cao [35] e, posteriormente, em outros trabalhos envolvendo crescimento crítico exponencial (ver [10], [12]).

Uma extensa pesquisa sobre problemas que apresentam uma perturbação do termo crítico tem sido desenvolvida desde o famoso artigo Brezis \& Niremberg [33]. Tais problemas surgem em diversos contextos. Citamos a seguir alguns exemplos que nos motivaram a analisar os problemas $\left(P_{2}\right)$ e $\left(P_{3}\right)$.

Em [73], Tarantello provou a existência de duas soluções para o problema em domínio limitado $\Omega \subset \mathbb{R}^{N}, N \geq 3$, dado por

$$
\left\{\begin{array}{l}
-\Delta u=|u|^{2^{*}-2} u+g(x), \quad x \in \Omega, \\
u=0, \quad x \in \partial \Omega
\end{array}\right.
$$

onde $g \in\left(W_{0}^{1,2}(\Omega)\right)^{\prime}$ e satisfaz algumas condições. 
Posteriormente, em [8], Alves também estabeleceu a existência de duas soluções para a equação mais geral

$$
-\Delta_{p} u=\lambda h u^{q}+|u|^{p^{*}-2} u, \quad u>0, \quad \mathbb{R}^{N}(N \geq 3)
$$

onde $p^{*}=\frac{N p}{N-p}$ é o expoente crítico de Sobolev, $2 \leq p<N, 0<q<p-1$ e $0 \leq h(x) \in L^{\Theta}$, $\Theta=\frac{N p}{N p-(q-1)(N-p)}$.

Para outras referências relacionadas aos problemas $\left(P_{2}\right)$ e $\left(P_{3}\right)$, com $g$ apresentando crescimento polinomial, citamos os autores Ambrosetti, Brezis \& Cerami [13], Pan [62], Cao, Li \& Zhou [36] e Gonçalves \& Alves [51]. A seguir, também mencionamos alguns trabalhos recentes para $g$ admitindo crescimento exponencial.

Em [28], Bezerra do Ó, Medeiros \& Severo, estudaram a classe de equações

$$
-\Delta u+V(x) u=g(u)+h(x), \quad x \in \mathbb{R}^{2},
$$

onde $V$ é um potencial contínuo satisfazendo $V(x) \geq V_{0}>0,[V(x)]^{-1} \in L^{1}\left(\mathbb{R}^{2}\right)$ e $h \in\left(W^{1,2}\left(\mathbb{R}^{2}\right)\right)^{\prime}$. No caso em que $g$ possui crescimento subcrítico exponencial os autores provaram a existência de pelo menos duas soluções, para $0<\|h\|<\delta$. Para o caso crítico foi obtida uma solução com energia negativa. Os mesmos autores provaram em [29], a existência de duas soluções para o problema

$$
-\Delta_{N} u+V(x)|u|^{N-2} u=g(x, u)+\epsilon h(x), \quad x \in \mathbb{R}^{N}(N \geq 2),
$$

onde $g$ possui crescimento crítico exponencial, $h \in\left(W^{1, N}\left(\mathbb{R}^{N}\right)\right)^{\prime}, h \neq 0$ e $\epsilon>0$.

Nosso objetivo é obter pelo menos duas soluções positivas para os problemas $\left(P_{2}\right)$ e $\left(P_{3}\right)$. Para isto, aplicamos o Princípio Variacional de Ekeland e uma versão do Teorema do Passo da Montanha sem a condição Palais-Smale. Além disso, adaptamos algumas idéias desenvolvidas em Alves [8], Alves, do Ó \& Miyagaki [10] e Alves \& Figueiredo [12].

Enunciamos agora os resultados de multiplicidade de soluções positivas para os problemas $\left(P_{2}\right)$ e $\left(P_{3}\right)$.

Teorema 2 Suponha que g satisfaz $\left(H_{c}\right),\left(H_{0}\right),\left(H_{4}\right)$ e $\left(H_{5}\right)$. Então, existe uma constante $\Lambda>0$ tal que, para $0<\lambda<\Lambda$, o problema $\left(P_{2}\right)$ possui pelo menos duas soluções positivas. 
Teorema 3 Suponha que g satisfaz $\left(H_{c}\right),\left(H_{0}\right),\left(H_{4}\right),\left(H_{5}\right),\left(H_{6}\right)$ e $\left(H_{7}\right)$. Então, existe uma constante $\Lambda>0$ tal que, para $0<\lambda<\Lambda$, o problema $\left(P_{3}\right)$ possui pelo menos duas soluções positivas.

Ainda no capítulo 2, provamos a existência de uma quantidade infinita de soluções (não necessáriamente positivas) para os problemas $\left(P_{2}\right)$ e $\left(P_{3}\right)$, no caso em que $g$ é ímpar. A técnica que utilizamos foi desenvolvida por Azorero \& Peral, em [17], com o estudo do problema em domínio limitado

$$
\left\{\begin{array}{l}
-\Delta_{p} u=|u|^{p^{*}-2} u+\lambda|u|^{q-2} u, \quad x \in \Omega, \\
u=0, \quad x \in \partial \Omega .
\end{array}\right.
$$

Os autores provaram a existência de solução, se $p<q<p^{*}$ e $\lambda$ é suficientemente pequeno ou se $\max \left\{p, p^{*}-\frac{p}{p-1}\right\}<q<p^{*}$. Quando $1<q<p$, o problema 0.6 possui uma quantidade infinita de soluções. Neste último caso, os autores usaram uma técnica que consiste em trabalhar com uma forma truncada do funcional energia associado, cujos pontos críticos são soluções do problema. Além disso, são aplicados resultados da Teoria do Gênero para obtenção de tais pontos críticos.

Utilizando este método, os autores Bonder \& Rossi [31] (ver também [25]), provaram o mesmo resultado para o problema de quarta ordem com condições de fronteira não linear

$$
\left\{\begin{array}{l}
-\Delta^{2} u=u, \quad \Omega \subset \mathbb{R}^{N}(N>4) \\
-\frac{\partial \Delta u}{\partial \eta}=|u|^{2_{*}-1} u+\lambda|u|^{q-1} u, \quad \frac{\partial u}{\partial \eta}=0 \quad \partial \Omega
\end{array}\right.
$$

onde $0<q<1,2_{*}=\frac{2(N-1)}{N-4}$ é o expoente crítico da imersão $W^{2,2}(\Omega) \hookrightarrow L^{2 *}(\partial \Omega)$.

Motivados por [17], provaremos no Capítulo 2 os seguintes resultados de multiplicidade para $\left(P_{2}\right)$ e $\left(P_{3}\right)$.

Teorema 4 Suponha que g é uma função ímpar que satisfaz $\left(H_{c}\right),\left(H_{0}\right),\left(H_{4}\right)$ e $\left(H_{5}\right)$. Então, existe uma constante $\hat{\Lambda}>0$ tal que, para $0<\lambda<\hat{\Lambda}$, o problema $\left(P_{2}\right)$ possui uma quantidade infinita de soluções. 
Teorema 5 Suponha que g é uma função ímpar que satisfaz $\left(H_{c}\right),\left(H_{0}\right),\left(H_{4}\right),\left(H_{5}\right),\left(H_{6}\right)$ $e\left(H_{7}\right)$. Então, existe uma constante $\hat{\Lambda}>0$ tal que, para $0<\lambda<\hat{\Lambda}$, o problema $\left(P_{3}\right)$ possui uma quantidade infinita de soluções.

No Capítulo 3, estudamos inicialmente a classe de problemas elípticos

$$
\left\{\begin{array}{l}
-\Delta_{N} u=a(x)|u|^{N-2} u+\lambda f(x, u)+g(x, u), \quad x \in \Omega, \\
u=0, \quad x \in \partial \Omega
\end{array}\right.
$$

onde $\Omega \subset \mathbb{R}^{N}$ é um domínio limitado com fronteira suave, $N \geq 2, \lambda>0$ e $a \in L^{\infty}(\Omega)$.

Hipóteses similares a $\left(H_{c}\right),\left(H_{0}\right),\left(H_{4}\right)$ e $\left(H_{5}\right)$ serão consideradas sobre $f$, devemos apenas adequá-las ao caso em que a função está definida em $\Omega \times \mathbb{R}$, mudaremos suas notações para $\left(\hat{H}_{c}\right),\left(\hat{H}_{0}\right),\left(\hat{H}_{4}\right)$ e $\left(\hat{H}_{5}\right)$, respectivamente. No entanto, para a função $g$, a condição $\left(H_{c}\right)$ será substituída pela seguinte condição de crescimento subcrítico exponencial:

$\left(\hat{\mathbf{H}}_{\mathbf{s c}}\right)$ dado $\beta>0$, existe $C_{\beta}>0$ tal que

$$
|g(x, s)| \leq C_{\beta} e^{\beta|s|^{\frac{N}{N-1}}}, \quad \forall s \in \mathbb{R}, \quad x \in \Omega .
$$

Outra hipótese a ser considerada para o estudo do problema $\left(P_{4}\right)$ é a seguinte:

$\left(\hat{\mathbf{H}}_{\mathbf{a}}\right)$ existe $A>0$ tal que

$$
\int_{\Omega}\left(|\nabla u|^{N}-a(x)|u|^{N}\right) d x \geq A \int_{\Omega}|u|^{N} d x, \quad \forall u \in W_{0}^{1, N}(\Omega) .
$$

Para estabelecer um resultado de multiplicidade para o problema $\left(P_{4}\right)$, aplicaremos uma versão do Teorema do Passo da Montanha de Ambrosetti-Rabinowitz (ver [66], pág. 55) que, para uma melhor compreensão deste texto, será demonstrada no Capítulo 3 com as adaptações necessárias. Além disso, extraímos algumas idéias contidas em Zhihui \& Xinmin [83], no qual os autores estudaram o problema $\left(P_{4}\right)$ com $f$ sendo uma potência polinomial crítica e $g$ uma perturbação subcrítica. Mais precisamente, Zhihui \& Xinmin consideraram o problema

$$
\left\{\begin{array}{l}
-\Delta_{p} u=a(x)|u|^{N-2} u+\lambda|u|^{p^{*}-2} u+g(x, u), \quad x \in \Omega \\
u=0, \quad x \in \partial \Omega
\end{array}\right.
$$

onde $1<p<N$. 
No estudo do problema 0.8 , os autores assumiram a hipótese $\left(\hat{H}_{a}\right)$ e consideraram outra versão do Teorema de Ambrosetti-Rabinowitz, para provar que existe uma sequência $\left(\lambda_{k}\right) \mathrm{com}$

$$
\lambda_{0}>\lambda_{1}>\ldots>\lambda_{k}>\ldots>0
$$

tal que, para $\lambda \in\left(\lambda_{k}, \lambda_{k-1}\right)$, o problema 0.8 possui pelo menos $k$ pares de soluções. No teorema seguinte, provamos a nossa versão deste resultado para o caso $p=N$ e com não linearidades admitindo um crescimento exponencial.

Teorema 6 Seja $f$ uma função impar em u satisfazendo as hipóteses $\left(\hat{H}_{c}\right),\left(\hat{H}_{0}\right),\left(\hat{H}_{4}\right)$ e $\left(\hat{H}_{5}\right)$. Seja g uma função impar em u satisfazendo $\left(\hat{H}_{s c}\right),\left(\hat{H}_{0}\right),\left(\hat{H}_{4}\right)$ e $\left(\hat{H}_{5}\right)$. Se a hipótese $\left(\hat{H}_{a}\right)$ ocorre, então existe uma sequência

$$
0<\lambda_{1}<\lambda_{2}<\ldots<\lambda_{k}<\ldots
$$

com $\lambda_{k} \rightarrow \infty$ quando $k \rightarrow \infty$, de modo que, para $\lambda>\lambda_{k}$, o problema $\left(P_{4}\right)$ possui pelo menos $k$ pares de soluções.

Outros resultados relacionados ao problema $\left(P_{4}\right)$ são encontrados, por exemplo, em Xavier [82] e Guedda \& Veron [48], Egnell [45].

Finalizamos o terceiro capítulo considerando o problema

$$
\left\{\begin{array}{c}
-\Delta_{N} u=\lambda f(|x|, u), \quad x \in \Omega_{r}, \\
u>0, \quad x \in \Omega_{r}, \\
u=0, \quad x \in \partial \Omega_{r},
\end{array}\right.
$$

onde $\Omega_{r}=\left\{x \in \mathbb{R}^{N} ; r<|x|<r+1\right\}$, com $N \geq 2, N \neq 3, r>0$ e $\lambda$ um parâmetro positivo.

Para este problema, vamos assumir as hipóteses $\left(\hat{H}_{c}\right),\left(\hat{H}_{0}\right),\left(\hat{H}_{4}\right)$ e $\left(\hat{H}_{5}\right)$ sobre a função $f$ e complementando estas hipóteses admitimos que $f$ satisfaz

$\left(\hat{\mathbf{H}}_{\mathbf{8}}\right)$ existem $\sigma \geq N$ e uma constante $C>0$ tais que

$$
\frac{\partial f}{\partial s}(|x|, s) s-(N-1) f(|x|, s) \geq C s^{\sigma}, \quad \forall s \geq 0 \quad \text { e } \forall x \in \Omega_{r} .
$$


Para uma breve motivação a respeito do problema $\left(P_{5}\right)$, iniciamos considerando o problema

$$
\left\{\begin{array}{l}
-\Delta u+u-u^{p}=0, \quad x \in D, \\
u=0, \quad x \in \partial D .
\end{array}\right.
$$

De acordo com Gidas, Ni \& Nirenberg [49], quando $D \subset \mathbb{R}^{N}$ é a bola unitária e $1<p<2^{*}-1$, se $N \geq 3$ ou $p>1$, se $N=2$, qualquer solução positiva de classe $C^{2}$ de $(0.9)$ deve ser radialmente simétrica. No entanto, se $D$ é um anel, digamos

$$
D=\left\{x \in \mathbb{R}^{N} ; r^{2}<|x|^{2}<(r+d)^{2}\right\}
$$

temos um fenômeno conhecido como quebra de simetria observado por Brezis \& Niremberg [33]. Mais precisamente, em [33] os autores provaram que para $N \geq 3$ existem soluções positivas não radiais de [0.9). Coffman [40], foi mais além, provando que o número de soluções positivas não radiais e não rotacionalmente equivalentes de $(0.9)$ em $D$, tende a $+\infty$ quando $r$ tende a $+\infty$, no caso $N=2, p>1$ ou $N \geq 3$ sendo par e $1<p<\frac{N}{N-2}$.

Motivados por estes trabalhos, outros autores deram suas contribuições no estudo de equações elípticas sobre anéis. Citamos os artigos Li [54], Suzuki [72] e Lin [55], para o caso em que $p$ é uma potência subcrítica.

No caso crítico, o seguinte problema foi considerado por Wang \& Willem [79]

$$
\left\{\begin{array}{l}
-\Delta u=\lambda u+u^{2^{*}-1}, u>0, \quad x \in \Omega_{r}, \\
u=0, \quad x \in \partial \Omega_{r},
\end{array}\right.
$$

onde $\Omega_{r}=\left\{x \in \mathbb{R}^{N} ; r<|x|<r+1\right\}, N \geq 4$. Os autores provaram que para $0<\lambda<\pi^{2}$ e $n \geq 1$, existe $R(\lambda, n)$ tal que, para $r>R(\lambda, n)$, a equação 0.10 possui pelo menos $n$ soluções não radiais e não rotacionalmente equivalentes.

Ainda para o caso crítico, Figueiredo \& Miyagaki [43] obtiveram resultados similares para o problema

$$
\left\{\begin{array}{l}
-\Delta u=f(|x|, u)+u^{2^{*}-1}, u>0, \quad x \in \Omega_{r}, \\
u=0, \quad x \in \partial \Omega_{r} .
\end{array}\right.
$$

Resultados para o caso $N=3$, podem ser encontrados, por exemplo, nos artigos Byeon [34, Castro \& Finan [37], Catrina \& Wang [38], Mizoguchi \& Suzuki [57], Hirano \& Mizoguchi [52] e suas referências. 
Finalizamos o Capítulo 3, estabelecendo o seguinte resultado de multiplicidade para o problema $\left(P_{5}\right)$.

Teorema 7 Suponha que $f$ é uma função satisfazendo $\left(\hat{H}_{c}\right),\left(\hat{H}_{0}\right),\left(\hat{H}_{4}\right),\left(\hat{H}_{5}\right)$ e $\left(\hat{H}_{8}\right)$. Então, para cada $n \in \mathbb{N}$, existem $\lambda(n), r(n)>0$ tais que, para $\lambda \geq \lambda(n)$ e $r \geq r(n)$, o problema $\left(P_{5}\right)$ possui pelo menos $n$ soluções não radiais e não rotacionalmente equivalentes.

No Capitulo 4, estudamos um resultado de existência e multiplicidade de soluções para a classe de problemas elípticos

$$
\left\{\begin{array}{l}
-\Delta_{N} u+|u|^{N-2} u=g(u), \quad x \in \Omega_{\lambda}, \\
u>0, \quad x \in \Omega_{\lambda} \\
u=0, \quad x \in \partial \Omega_{\lambda},
\end{array}\right.
$$

onde $\Omega_{\lambda}=\lambda \Omega$ com $\Omega \subset \mathbb{R}^{N}, N \geq 2$, sendo um domínio limitado e $\lambda>1$.

Vamos supor que $g$ satifaz as hipóteses $\left(H_{c}\right),\left(H_{0}\right),\left(H_{4}\right),\left(H_{5}\right),\left(H_{6}\right)$ e adicionamos a seguinte hipótese:

$\left(\mathbf{H}_{\mathbf{8}}\right)$ existem $\sigma \geq N$ e uma constante $C>0$ tais que

$$
g^{\prime}(s) s-(N-1) g(s) \geq C s^{\sigma}, \quad \forall s \geq 0 .
$$

Aplicando resultados envolvendo Categoria de Lusternik-Schnirelman, seremos capazes de provar a existência de pelo menos a categoria de $\Omega_{\lambda}$ em $\Omega_{\lambda}$ (ver Apêndice) de soluções positivas para o problema $\left(P_{6}\right)$, para $\lambda$ suficientemente grande.

Em [19], Benci \& Cerami provaram que, para $2<p<2^{*}$ e $\lambda$ grande, existe pelo menos $\operatorname{cat}_{\Omega}(\Omega)$ de soluções para o problema

$$
\left\{\begin{array}{l}
-\Delta u+\lambda u=u^{p-1}, \quad u>0, \quad x \in \Omega \subset \mathbb{R}^{N}(N \geq 3), \\
u=0, \quad x \in \partial \Omega .
\end{array}\right.
$$

Posteriormente, os mesmos autores em [21], consideraram o problema

$$
\left\{\begin{array}{l}
-\epsilon \Delta u+u=f(u), \quad u>0, \quad x \in \Omega \\
u=0, \quad x \in \partial \Omega
\end{array}\right.
$$


onde $\epsilon>0, \Omega \subset \mathbb{R}^{N}(N \geq 3)$ satisfazendo $\operatorname{cat}_{\Omega}(\Omega)>1$ e $f \in C^{1,1}\left(\mathbb{R}^{+}, \mathbb{R}\right)$ verificando algumas hipóteses. Usando Categoria de Lusternik-Schnirelman, os autores provaram que para $\epsilon$ suficientemente pequeno, o problema 0.12 tem pelo menos $\operatorname{cat}_{\Omega}(\Omega)+1$ de soluções distintas. Estes resultados, foram obtidos também em Alves [7], para o seguinte problema

$$
\left\{\begin{array}{l}
-\Delta_{p} u+|u|^{p-2} u=g(u), \quad x \in \Omega_{\lambda}, \\
u>0, \quad x \in \Omega_{\lambda}, \\
u=0, \quad x \in \partial \Omega_{\lambda},
\end{array}\right.
$$

onde $2 \leq p<N$ e $g$ é uma função com crescimento subcrítico no infinito.

Ampliando posteriormente este estudo, Alves \& Figueiredo [12], estabeleceram um resultado de multiplicidade de soluções positivas, utilizando a Categoria de LusternikSchnirelman, para o problema quasilinear com crescimento crítico exponencial em $\mathbb{R}^{N}$

$$
-\epsilon^{N} \Delta_{N} u+V(x)|u|^{N-2} u=g(u), \quad x \in \mathbb{R}^{N}
$$

com $V: \mathbb{R}^{N} \rightarrow \mathbb{R}$ uma função contínua.

Para um estudo mais detalhado sobre resultados de multiplicidade envolvendo Categoria de Lusternik-Schnirelman podemos sugerir os artigos Cerami \& Passasseo [39], Alves \& Ding [11], Rey [67] e Bahri \& Coron [18].

Iremos explorar algumas idéias e fatos contidos em [12] e em [7] para demonstrar o resultado principal do Capítulo 4.

Teorema 8 Suponha que g satisfaz $\left(H_{c}\right),\left(H_{0}\right),\left(H_{4}\right),\left(H_{5}\right),\left(H_{6}\right)$ e $\left(H_{8}\right)$. Então, existe $\lambda^{*}>0$ tal que, para $\lambda>\lambda^{*}$, o problema $\left(P_{6}\right)$ possui pelo menos cat $\Omega_{\Omega_{\lambda}}\left(\Omega_{\lambda}\right)$ de soluções.

Para o leitor interessado no estudo de problemas envolvendo o operador $N$-Laplaciano e não linearidades com crescimento exponencial, indicamos, além das referências já citadas, os artigos Silva \& Soares [70], Wang, Yang \& Zhang [80], Tonkes [76] e Panda 63]. 


\section{CAPÍTULO 1}

\section{EXISTÊNCIA DE SOLUÇÃO PARA UM PROBLEMA}

\section{INDEFINIDO EM DOMÍNIO EXTERIOR}

Neste capítulo, vamos estabelecer a existência de solução para o problema

$$
\left\{\begin{array}{l}
-\Delta_{N} u+|u|^{N-2} u=a(x) g(u), \quad x \in \Omega, \\
u=0, \quad x \in \partial \Omega,
\end{array}\right.
$$

onde $\Omega=\mathbb{R}^{N} \backslash \Theta$, com $N \geq 2$ e $\Theta \subset \mathbb{R}^{N}$ sendo um domínio limitado com fronteira suave. Vamos supor que a função $a(x)$ satisfaz as seguintes hipóteses:

$\left(\mathbf{a}_{\mathbf{1}}\right) a: \Omega \rightarrow \mathbb{R}$ é uma função contínua que muda de sinal em $\Omega$;

$\left(\mathbf{\mathbf { a } _ { 2 }}\right) \operatorname{dist}\left(\overline{\Omega^{+}}, \overline{\Omega^{-}}\right)=\delta>0$, onde $\Omega^{+}=\{x \in \Omega ; a(x)>0\}, \quad \Omega^{-}=\{x \in \Omega ; a(x)<0\} \mathrm{e}$ dist é a função distância entre conjuntos;

(a) $a \in L^{\infty}\left(\Omega^{-}\right)$e existe $R>0$ tal que $a(x)<0$, para $|x| \geq R$.

Assumimos que $g$ satisfaz a condição de crescimento crítico exponencial

$\left(\mathbf{H}_{\mathbf{c}}\right)$ existe $\alpha_{0}>0$ tal que

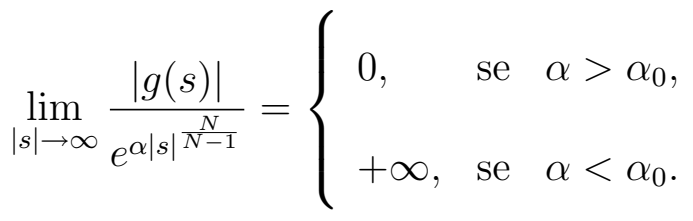


Admitimos também que $g$ verifica

$\left(\mathbf{H}_{\mathbf{0}}\right) g: \mathbb{R} \rightarrow \mathbb{R}$ é uma função contínua tal que

$$
g(s)=o\left(|s|^{N-1}\right), \text { quando } s \rightarrow 0 .
$$

Observe que se a condição $\left(a_{2}\right)$ é satisfeita, existe uma função $\zeta \in C^{\infty}(\Omega)$ tal que

$$
\begin{gathered}
0 \leq \zeta(x) \leq 1, \quad x \in \Omega, \\
\zeta(x)=1, \quad x \in \Omega^{+}, \quad \zeta(x)=0, \quad x \in \Omega^{-}
\end{gathered}
$$

e

$$
M:=\sup _{\Omega}|\nabla \zeta|<\infty
$$

A função $\zeta$ será utilizada para formular a seguinte versão da condição de AmbrosettiRabinowitz:

$\left(\mathbf{H}_{\mathbf{1}}\right)$ existem $\nu>0$ e $0<\theta<\frac{\nu}{N+(N-1) M}$ tais que

$$
0<\frac{\nu}{\theta} G(s) \leq g(s) s, \quad \forall|s|>0,
$$

onde $G(s)=\int_{0}^{s} g(t) d t$

Para finalizar as hipóteses, assumimos que $g$ cumpre as seguintes condições de crescimento:

$\left(\mathbf{H}_{\mathbf{2}}\right)$ existem constantes $K_{0}, R_{0}>0$ tais que

$$
0<G(s) \leq K_{0}|g(s)|, \quad \forall|s| \geq R_{0}
$$

$\left(\mathbf{H}_{\mathbf{3}}\right) \lim _{s \rightarrow \infty} s g(s) e^{-\alpha_{0}|s|^{\frac{N}{N-1}}}=+\infty$.

Observação 1.1 Um exemplo típico de uma função que verifica as hipóteses $\left(H_{c}\right),\left(H_{0}\right)-$ $\left(H_{3}\right)$ é a função $g: \mathbb{R} \rightarrow \mathbb{R}$ definida por

$$
g(s)=\left(q|s|^{q-2} s+\frac{\alpha_{0} N}{N-1}|s|^{q+\frac{N}{N-1}-2} s\right) e^{\alpha_{0}|s|^{\frac{N}{N-1}}}, \quad \text { onde } \quad q>\frac{\nu}{\theta}
$$

Observe que neste exemplo temos

$$
G(s)=|s|^{q} e^{\alpha_{0}|s|^{\frac{N}{N-1}}} .
$$


Com o propósito de obter um resultado de existência de solução para o problema $\left(P_{1}\right)$ faremos uso do Teorema do Passo da Montanha sem a condição Palais-Smale e de uma versão da Desigualdade de Trudinger-Moser.

Este capítulo está organizado da seguinte forma: Na primeira seção introduzimos alguns resultados preliminares e a formulação variacional do problema. Além disso, verificamos as hipóteses do Teorema do Passo da Montanha e mostramos uma importante estimativa envolvendo o nível do Passo da Montanha. Na Seção 1.2 provamos algumas propriedades da sequência Palais-Smale obtida do Teorema do Passo da Montanha. Em seguida, na Seção 1.3 , provamos o Teorema 1 .

\section{$1.1 \quad$ Preliminares}

Iniciamos esta seção apresentando uma versão da Desigualdade de Trudinger-Moser para domínios ilimitados que pode ser encontrada em Bezerra do Ó [27] (ver também Cao [35]).

\section{Lema 1.1 (Desigualdade de Trudinger-Moser para o $\mathbb{R}^{N}$ )}

Dada qualquer $u \in W^{1, N}\left(\mathbb{R}^{N}\right)$ com $N \geq 2$, temos que

$$
\int_{\mathbb{R}^{N}}\left(e^{\alpha|u|^{N-1}}-S_{N-2}(\alpha, u)\right) d x<\infty
$$

para cada $\alpha>0$. Além disso, se $|\nabla u|_{N}^{N} \leq 1,|u|_{N} \leq K<\infty$ e $\alpha<\alpha_{N}=N \omega_{N-1}^{\frac{1}{N-1}}$, então existe uma constante positiva $C=C(N, K, \alpha)$ tal que

$$
\int_{\mathbb{R}^{N}}\left(e^{\alpha|u|^{N-1}}-S_{N-2}(\alpha, u)\right) d x \leq C
$$

onde

$$
S_{N-2}(\alpha, u)=\sum_{k=0}^{N-2} \frac{\alpha^{k}}{k !}|u|^{\frac{N k}{N-1}}
$$

e $\omega_{N-1}$ é a medida $(N-1)$-dimensional da $(N-1)$-esfera. 
Lema 1.2 Sejam $\alpha>0$ e $r>1$. Então, para cada $\beta>r$, existe uma constante $C=C(\beta)>0$ tal que

$$
\left(e^{\alpha|s|^{\frac{N}{N-1}}}-S_{N-2}(\alpha, s)\right)^{r} \leq C\left(e^{\beta \alpha|s|^{N-1}}-S_{N-2}(\beta \alpha, s)\right) .
$$

Demonstração. Para simplificar as notações, escreveremos

$$
y:=|s|^{\frac{N}{N-1}} \quad \text { e } \quad \tilde{S}(\alpha, y)=\sum_{k=0}^{N-2} \frac{\alpha^{k} y^{k}}{k !} .
$$

Observe que

$$
\frac{\left(e^{\alpha y}-\tilde{S}(\alpha, y)\right)^{r}}{\left(e^{\beta \alpha y}-\tilde{S}(\beta \alpha, y)\right)}=\frac{\left(\sum_{k=N-1}^{\infty} \frac{\alpha^{k} y^{k}}{k !}\right)^{r}}{\sum_{k=N-1}^{\infty} \frac{(\beta \alpha)^{k} y^{k}}{k !}}=\frac{y^{r(N-1)}\left(\sum_{k=N-1}^{\infty} \frac{\alpha^{k} y^{k-N+1}}{k !}\right)^{r}}{y^{N-1} \sum_{k=N-1}^{\infty} \frac{(\beta \alpha)^{k} y^{k-N+1}}{k !}},
$$

de onde deduzimos que

$$
\lim _{y \rightarrow 0} \frac{\left(e^{\alpha y}-\tilde{S}(\alpha, y)\right)^{r}}{\left(e^{\beta \alpha y}-\tilde{S}(\beta \alpha, y)\right)}=0
$$

Além disso,

$$
\lim _{y \rightarrow \infty} \frac{\left(e^{\alpha y}-\tilde{S}(\alpha, y)\right)^{r}}{\left(e^{\beta \alpha y}-\tilde{S}(\beta \alpha, y)\right)}=\frac{e^{r \alpha y}\left(1-\frac{\tilde{S}(\alpha, y)}{e^{\alpha y}}\right)^{r}}{e^{\beta \alpha y}\left(1-\frac{\tilde{S}(\beta \alpha, y)}{e^{\alpha y}}\right)}=0
$$

provando o lema.

No espaço de Sobolev $W_{0}^{1, N}(\Omega)$ consideramos a norma dada por

$$
\|u\|=\left[\int_{\Omega}\left(|\nabla u|^{N}+|u|^{N}\right) d x\right]^{1 / N}, \quad u \in W_{0}^{1, N}(\Omega),
$$

e denotamos por $I: W_{0}^{1, N}(\Omega) \rightarrow \mathbb{R}$, o funcional energia associado ao problema $\left(P_{1}\right)$, que é definido por

$$
I(u)=\frac{1}{N}\|u\|^{N}-\int_{\Omega} a(x) G(u) d x
$$

Observação 1.2 As hipóteses $\left(H_{c}\right),\left(H_{0}\right),\left(a_{1}\right)$ e $\left(a_{3}\right)$, juntamente com a Desigualdade de Trudinger-Moser, asseguram que

$$
\int_{\Omega} a(x) G(u) d x<\infty
$$

Portanto, o funcional I está bem definido. 
Um fato importante, que pode ser encontrado em Bezerra do Ó [29], é que para qualquer sequência $\left(u_{n}\right)$ em $W^{1, N}\left(\mathbb{R}^{N}\right)$ fortemente convergente, existem uma subsequência $\left(u_{n_{k}}\right)$ e uma função $v \in W^{1, N}\left(\mathbb{R}^{N}\right)$ verificando

$$
\left|u_{n_{k}}(x)\right| \leq v(x), \quad \text { q. t. p. em } \mathbb{R}^{N}
$$

Com esta informação podemos adaptar as idéias encontradas em [66] (Apêndice B) (ver também [24], Apêndice) ao caso exponencial e, assim, justificar que o funcional energia I pertence a $C^{1}\left(W_{0}^{1, N}(\Omega), \mathbb{R}\right)$. Além disso,

$$
I^{\prime}(u) v=\int_{\Omega}\left(|\nabla u|^{N-2} \nabla u \nabla v+|u|^{N-2} u v\right) d x-\int_{\Omega} a(x) g(u) v d x, \quad \forall u, v \in W_{0}^{1, N}(\Omega) .
$$

Consequentemente, cada ponto crítico de I é uma solução do problema $\left(P_{1}\right)$.

Veremos no lema a seguir que, para qualquer sequência verificando

$$
\limsup _{n \rightarrow \infty}\left\|u_{n}\right\|^{N}<\left(\frac{\alpha_{N}}{\alpha_{0}}\right)^{N-1}
$$

podemos obter $\alpha>\alpha_{0}$ e $t>1$ tais que a sequência

$$
Q_{n}:=\left[e^{\alpha\left|u_{n}\right|^{N-1}}-S_{N-2}\left(\alpha, u_{n}\right)\right]
$$

é limitada em $L^{t}(\Omega)$. Este fato é uma consequência da Desigualdade de TrudingerMoser (Lema 1.1) e será de fundamental importância para provar algumas convergências envolvendo sequências Palais-Smale. Nos próximos capítulos também iremos recorrer a este resultado.

Lema 1.3 Seja $\left(u_{n}\right)$ uma sequência satisfazendo (1.1). Então, existem $\alpha>\alpha_{0}, t>1 e$ $C>0$, independente de $n$, tais que

$$
\int_{\Omega}\left[e^{\alpha\left|u_{n}\right|^{\frac{N}{N-1}}}-S_{N-2}\left(\alpha, u_{n}\right)\right]^{t} d x \leq C, \quad \forall n \geq n_{0}
$$

para algum $n_{0}$ suficientemente grande. 
Demonstração. Observe que a hipótese garante que existem $m>0$ e $n_{0} \in \mathbb{N}$ tais que

$$
\left\|u_{n}\right\|^{\frac{N}{N-1}}<m<\frac{\alpha_{N}}{\alpha_{0}}, \quad \forall n \geq n_{0} .
$$

Escolha $\alpha>\alpha_{0}, t>1$ e $\beta>t$ satisfazendo $\alpha m<\alpha_{N}, t \alpha m<\alpha_{N}$ e $\beta \alpha m<\alpha_{N}$. Pelo Lema 1.2 , existe $C=C(\beta)$ tal que

$$
\int_{\Omega}\left[e^{\alpha\left|u_{n}\right|^{N-1}}-S_{N-2}\left(\alpha, u_{n}\right)\right]^{t} d x \leq C \int_{\mathbb{R}^{N}}\left[e^{\beta \alpha m\left(\frac{\left|u_{n}\right|}{|| u_{n}||}\right)^{\frac{N}{N-1}}}-S_{N-2}\left(\beta \alpha m, \frac{\left|u_{n}\right|}{|| u_{n} \|}\right)\right] d x
$$

para cada $n \geq n_{0}$.

Aplicando a Desigualdade de Trudinger-Moser (Lema 1.1), existe uma constante $C>0$, independente de $n$, tal que

$$
\int_{\Omega}\left[e^{\alpha\left|u_{n}\right|^{\frac{N}{N-1}}}-S_{N-2}\left(\alpha, u_{n}\right)\right]^{t} d x \leq C, \quad \forall n \geq n_{0},
$$

como queríamos demonstrar.

No próximo lema, provamos que o funcional $I$ satisfaz a geometria do Passo da Montanha.

Lema 1.4 O funcional energia I verifica as seguintes condições:

(i) Existem $\beta, \rho>0$ tais que

$$
I(u) \geq \beta, \quad \text { para }\|u\|=\rho .
$$

(ii) Existe $e \in W_{0}^{1, N}(\Omega)$ tal que $\|e\|>\rho$ e $I(e)<0$.

Demonstração. Por $\left(H_{0}\right)$ e $\left(H_{c}\right)$, temos que dados $\epsilon>0$ e $q \geq 1$, existe $C=C(\epsilon, q)>0$ tal que, para cada $\alpha>\alpha_{0}$,

$$
|g(t)| \leq \epsilon|t|^{N-1}+C|t|^{q-1}\left(e^{\alpha|t|^{\frac{N}{N-1}}}-S_{N-2}(\alpha, t)\right), \quad \forall t \in \mathbb{R} .
$$

Consequentemente,

$$
|G(t)| \leq \frac{\epsilon}{N}|t|^{N}+C|t|^{q}\left(e^{\alpha|t|^{\frac{N}{N-1}}}-S_{N-2}(\alpha, t)\right), \quad \forall t \in \mathbb{R}
$$


Segue da hipótese $\left(a_{3}\right)$ que $\Omega^{+}$é limitado e, assim, podemos considerar $C_{0}=\sup _{\Omega^{+}} a(x)$. Fixando $q>N$, obtemos

$$
\begin{aligned}
\int_{\Omega} a(x) G(u) d x & \leq \int_{\Omega^{+}} a(x)|G(u)| d x \\
& \leq C_{0} \frac{\epsilon}{N} \int_{\Omega^{+}}|u|^{N} d x+C_{0} C \int_{\Omega^{+}}|u|^{q}\left[e^{\alpha|u|^{N-1}}-S_{N-2}(\alpha, u)\right] d x
\end{aligned}
$$

e usando a Desigualdade de Hölder,

$$
\int_{\Omega} a(x) G(u) d x \leq \frac{\epsilon}{N} C_{0}|u|_{N}^{N}+C_{1}|u|_{q s_{1}}^{q}\left|e^{\alpha|u|^{N-1}}-S_{N-2}(\alpha, u)\right|_{t_{1}}
$$

onde $\frac{1}{s_{1}}+\frac{1}{t_{1}}=1$.

Pela definição de $I$, juntamente com $(1.2)$ e a imersão de Sobolev

$$
W_{0}^{1, N}(\Omega) \hookrightarrow L^{t}(\Omega), N \leq t<\infty
$$

obtemos constantes positivas $C_{2}$ e $C_{3}$ tais que

$$
I(u) \geq \frac{1}{N}\|u\|^{N}-\frac{\epsilon}{N} C_{2}\|u\|^{N}-C_{3}\|u\|^{q}\left|e^{\alpha|u|^{\frac{N}{N-1}}}-S_{N-2}(\alpha, u)\right|_{t_{1}} .
$$

Fixe $\rho_{0}>0$, de modo que $\alpha \rho_{0}^{\frac{N}{N-1}}<\alpha_{N}$. Podemos escolher $t_{1}>1$, suficientemente próximo de 1 , tal que $t_{1} \alpha \rho_{0}^{\frac{N}{N-1}}<\alpha_{N}$ e também escolher $\eta>t_{1}$ tal que $\eta \alpha \rho_{0}^{\frac{N}{N-1}}<\alpha_{N}$. Aplicando o Lema 1.2 , segue que para cada $u \in W_{0}^{1, N}(\Omega)$ com $\|u\| \leq \rho_{0}$, temos

$$
\begin{aligned}
& \left|e^{\alpha|u|^{\frac{N}{N-1}}}-S_{N-2}(\alpha, u)\right|_{t_{1}}=\left\{\int_{\Omega}\left[e^{\alpha|u|^{\frac{N}{N-1}}}-S_{N-2}(\alpha, u)\right]^{t_{1}} d x\right\}^{\frac{1}{t_{1}}} \\
& \leq C\left\{\int_{\Omega}\left[e^{\eta \alpha\|u\|^{\frac{N}{N-1}}\left(\frac{|u| \mid}{\|u\|}\right)^{\frac{N}{N-1}}}-S_{N-2}\left(\eta \alpha\|u\|^{\frac{N}{N-1}}, \frac{|u|}{\|u\|}\right)\right] d x\right\}^{\frac{1}{t_{1}}} \\
& \leq C\left\{\int_{\Omega}\left[e^{\eta \alpha \rho_{0}^{\frac{N}{N-1}}\left(\frac{|u|}{\|u\|}\right)^{\frac{N}{N-1}}}-S_{N-2}\left(\eta \alpha \rho_{0}^{\frac{N}{N-1}}, \frac{|u|}{\|u\|}\right)\right] d x\right\}^{\frac{1}{t_{1}}} .
\end{aligned}
$$

Portanto, a Desigualdade de Trudinger-Moser garante que existe uma constante $C_{4}>0$ tal que

$$
\left|e^{\alpha|u|^{\frac{N}{N-1}}}-S_{N-2}(\alpha, u)\right|_{t_{1}} \leq C_{4}, \quad \forall\|u\| \leq \rho_{0} .
$$

Substituindo (1.4) em (1.3), obtemos 


$$
I(u) \geq\left(\frac{1}{N}-\frac{\epsilon}{N} C_{2}\right)\|u\|^{N}-C_{5}\|u\|^{q} .
$$

Uma vez que $q>N$, temos que para $\epsilon>0$ suficientemente pequeno, existem $\beta>0$ e $0<\rho<\rho_{0}$ tais que

$$
I(u) \geq \beta>0, \quad \text { para }\|u\|=\rho,
$$

mostrando o item $(i)$.

Observe agora que por $\left(H_{1}\right)$, existem constantes $C, D>0$ verificando

$$
G(t) \geq C|t|^{\frac{\nu}{\theta}}-D, \quad \forall t \in \mathbb{R}
$$

Sendo $\Omega^{+} \neq \emptyset$, podemos fixar uma função $0 \leq \varphi \in C_{0}^{\infty}\left(\Omega^{+}\right)$. Assim, usando 1.5), encontramos

$$
\begin{aligned}
I(t \varphi) & =\frac{t^{N}}{N}\|\varphi\|^{N}-\int_{\text {supp } \varphi} a(x) G(t \varphi) d x \\
& \leq \frac{t^{N}}{N}\|\varphi\|^{N}-C|t|^{\frac{\nu}{\theta}} \int_{\text {supp } \varphi} a(x)|\varphi|^{\frac{\nu}{\theta}} d x+D \int_{\text {supp } \varphi} a(x) d x
\end{aligned}
$$

Visto que $\frac{\nu}{\theta}>N$, concluímos que $I(t \varphi) \rightarrow-\infty$ quando $t \rightarrow+\infty$. Assim, é suficiente fixar $e=t \varphi \operatorname{com} t>0$ suficientemente grande para concluir a prova.

Aplicando uma versão do Teorema do Passo da Montanha sem a condição Palais-Smale (ver Apêndice, Teorema A.6) garantimos a existência de uma sequência Palais-Smale no nível $c$, isto é, existe uma sequência $\left(u_{n}\right) \subset W_{0}^{1, N}(\Omega)$ satisfazendo

$$
I\left(u_{n}\right) \rightarrow c \quad \text { e } \quad I^{\prime}\left(u_{n}\right) \rightarrow 0, \quad \text { quando } n \rightarrow+\infty
$$

onde

$$
c=\inf _{\gamma \in \Gamma} \max _{t \in[0,1]} I(\gamma(t))
$$

com

$$
\Gamma=\left\{\gamma \in C\left([0,1], W_{0}^{1, N}(\Omega)\right) ; \gamma(0)=0 \text { e } \gamma(1)=e\right\},
$$

onde $e$ foi obtido no Lema 1.4 .

No próximo resultado, usamos as funções de Moser juntamente com a condição $\left(H_{3}\right)$, a fim de obter uma importante estimativa para o nível do Passo da Montanha. Antes disto, fixamos algumas notações. 
Sem perda de generalidade, suponha que $0 \in \Omega^{+}$e seja $B_{\tau}(0) \subset \subset \Omega^{+}$. Considere as funções de Moser (ver [60]):

$$
\tilde{M}_{n}(x)=1 / \omega_{N-1}^{\frac{1}{N}}\left\{\begin{array}{lll}
(\log n)^{\frac{N-1}{N}}, & \text { se } & |x| \leq \frac{\tau}{n} \\
\frac{\log \left(\frac{\tau}{|x|}\right)}{(\log n)^{\frac{1}{N}}}, & \text { se } & \frac{\tau}{n} \leq|x| \leq \tau \\
0, & \text { se } & |x| \geq \tau .
\end{array}\right.
$$

Segue da definição de $\tilde{M}_{n}(x)$, que valem as seguintes propriedades:

(i) $\tilde{M}_{n} \in W_{0}^{1, N}\left(\mathbb{R}^{N}\right)$ com suporte na bola $B_{\tau}(0)$;

(ii) $\int_{\mathbb{R}^{N}}\left|\nabla \tilde{M}_{n}(x)\right|^{N}=\int_{B_{\tau}(0)}\left|\nabla \tilde{M}_{n}(x)\right|^{N}=1 ;$

(iii) $\int_{\mathbb{R}^{N}}\left|\tilde{M}_{n}(x)\right|^{N}=O\left(\frac{1}{\log n}\right) \rightarrow 0$, quando $n \rightarrow+\infty$.

Considerando

$$
M_{n}(x)=\frac{\tilde{M}_{n}(x)}{\left\|\tilde{M}_{n}\right\|},
$$

temos que para $|x| \leq \tau / n$,

$$
M_{n}^{\frac{N}{N-1}}=\omega_{N-1}^{-1 /(N-1)} \log n\left\|\tilde{M}_{n}\right\|^{\frac{-N}{N-1}} .
$$

Uma vez que

$$
\omega_{N-1}^{-1 /(N-1)}=\left(\alpha_{N} / N\right)^{-1}=N / \alpha_{N}
$$

podemos escrever

$$
\begin{aligned}
M_{n}^{\frac{N}{N-1}} & =N / \alpha_{N} \log n\left\|\tilde{M}_{n}\right\|^{\frac{-N}{N-1}}+N / \alpha_{N} \log n-N / \alpha_{N} \log n \\
& =N / \alpha_{N} \log n+N / \alpha_{N} \log n\left(\left\|\tilde{M}_{n}\right\|^{\frac{-N}{N-1}}-1\right) .
\end{aligned}
$$

Denotando

$$
d_{n}:=\left(N / \alpha_{N} \log n\left(\left\|\tilde{M}_{n}\right\|^{\frac{-N}{N-1}}-1\right)\right)
$$

temos

$$
M_{n}^{\frac{N}{N-1}}=N / \alpha_{N} \log n+d_{n} .
$$

Note que pelas propriedades acima, segue que $\left\|\tilde{M}_{n}\right\| \rightarrow 1$, o que implica

$$
\frac{d_{n}}{\log n} \rightarrow 0
$$


Uma versão do lema seguinte pode ser encontrado em Bezerra do Ó, Medeiros \& Severo [29] (ver Lema 4.1) e a prova segue com argumentos similares.

Lema 1.5 O nível c do Passo da Montanha satisfaz

$$
c<\frac{1}{N}\left(\frac{\alpha_{N}}{\alpha_{0}}\right)^{N-1}
$$

Demonstração. Como vimos na demonstração do Lema 1.4 (ii), fixado $n \in \mathbb{N}$, podemos escolher $e=t_{0} M_{n}$, com $t_{0}>0$ suficientemente grande. Deste modo, considerando o caminho $\gamma(t)=t t_{0} M_{n}$, temos que $\gamma \in \Gamma$. Logo, pela definição de $c$,

$$
c \leq \max _{t \geq 0} I\left(t M_{n}\right), \quad \forall n \in \mathbb{N} .
$$

Assim, é suficiente mostrar que existe $n \in \mathbb{N}$ tal que

$$
\max _{t \geq 0} I\left(t M_{n}\right)<\frac{1}{N}\left(\frac{\alpha_{N}}{\alpha_{0}}\right)^{N-1} .
$$

Argumentando por contradição, suponha que

$$
\max _{t \geq 0} I\left(t M_{n}\right) \geq \frac{1}{N}\left(\frac{\alpha_{N}}{\alpha_{0}}\right)^{N-1}, \quad \forall n \in \mathbb{N}
$$

Logo,

$$
\max _{t \geq 0}\left\{\frac{t^{N}}{N}-\int_{B_{\tau}(0)} a(x) G\left(t M_{n}\right) d x\right\} \geq \frac{1}{N}\left(\frac{\alpha_{N}}{\alpha_{0}}\right)^{N-1}, \quad \forall n \in \mathbb{N}
$$

Segue da estimativa (1.5) que o máximo acima é atingido para cada $n \in \mathbb{N}$, ou seja, existe $t_{n}>0$ tal que

$$
\frac{t_{n}^{N}}{N}-\int_{B_{\tau}(0)} a(x) G\left(t_{n} M_{n}\right) d x=\max _{t \geq 0}\left\{\frac{t^{N}}{N}-\int_{B_{\tau}(0)} a(x) G\left(t M_{n}\right) d x\right\} \geq \frac{1}{N}\left(\frac{\alpha_{N}}{\alpha_{0}}\right)^{N-1} .
$$

Usando que $a(x) G(u)>0$ em $B_{\tau}(0)$, obtemos

$$
\frac{t_{n}^{N}}{N} \geq \frac{1}{N}\left(\frac{\alpha_{N}}{\alpha_{0}}\right)^{N-1}, \quad \forall n \in \mathbb{N},
$$

ou ainda,

$$
t_{n}^{N} \geq\left(\frac{\alpha_{N}}{\alpha_{0}}\right)^{N-1}, \quad \forall n \in \mathbb{N}
$$


Observe agora que

$$
\left.\frac{d}{d t}\left(\frac{t^{N}}{N}-\int_{B_{\tau}(0)} a(x) G\left(t M_{n}\right) d x\right)\right|_{t=t_{n}}=0
$$

ou seja,

$$
t_{n}^{N}=\int_{B_{\tau}(0)} a(x) g\left(t_{n} M_{n}\right)\left(t_{n} M_{n}\right) d x
$$

Da hipótese $\left(H_{3}\right)$, segue que dado $K>0$, existe $\eta=\eta(K)>0$ tal que

$$
u g(u) \geq K e^{\alpha_{0}|u|^{\frac{N}{N-1}}}, \quad \text { para } \quad|u|>\eta .
$$

Substituindo em (1.11), ficamos com

$$
t_{n}^{N} \geq \int_{|x| \leq \tau / n} a(x) g\left(t_{n} M_{n}\right)\left(t_{n} M_{n}\right) d x \geq K \int_{|x| \geq \tau / n} a(x) e^{\alpha_{0}\left|t_{n} M_{n}\right|^{\frac{N}{N-1}}} d x
$$

para $n$ suficientemente grande, onde aqui estamos usando a definição de $M_{n}$ em $|x|<\tau / n$.

Da desigualdade anterior e de (1.7), temos

$$
t_{n}^{N} \geq a_{0} K C_{N}\left(\frac{\tau}{n}\right)^{N} e^{\left(\frac{\alpha_{0}}{\alpha_{N}} N t_{n}^{\frac{N}{N-1}} \log n+\alpha_{0} t_{n}^{\frac{N}{N-1}} d_{n}\right)}
$$

onde $a_{0}=\min \left\{a(x) ; x \in B_{\tau}(0)\right\}$. Consequentemente,

$$
e^{N \log t_{n}} \geq a_{0} K C_{N} \tau^{N} e^{-N \log n} e^{\left(\frac{\alpha_{0}}{\alpha_{N}} N t_{n}^{\frac{N}{N-1}} \log n+\alpha_{0} t_{n}^{\frac{N}{N-1}} d_{n}\right)}
$$

ou ainda,

$$
\left.1 \geq K C_{N} \tau^{N} e^{\left(\frac{\alpha_{0}}{\alpha_{N}} N t_{n}^{\frac{N}{N-1}} \log n+\alpha_{0} t_{n}^{\frac{N}{N-1}}\right.} d_{n}-N \log t_{n}-N \log n\right),
$$

de onde segue que $\left(t_{n}\right)$ é limitada, pois, caso contrário, o lado direito tenderia a $+\infty$, chegando a uma contradição.

Usando a hipótese $\left(H_{3}\right)$ e repetindo os mesmos argumentos encontrados em [29] podemos provar que, para qualquer $K>0$, existe uma constante positiva $C_{\tau}$, independente de $K$, tal que

$$
\limsup _{n \rightarrow+\infty} t_{n} \geq C_{\tau} K
$$

Uma vez que $K$ é arbitrário e $\left(t_{n}\right)$ é limitada, a última desigualdade não pode ocorrer, assim, chegamos a uma contradição. 


\subsection{Propriedades da sequência Palais-Smale}

Nos próximos lemas provamos algumas propriedades da sequência Palais-Smale $\left(u_{n}\right)$ dada por 1.6 .

Lema 1.6 Seja $\left(u_{n}\right)$ a sequência satisfazendo (1.6). Então, $\left(u_{n}\right)$ é limitada em $W_{0}^{1, N}(\Omega)$.

Demonstração. Nesta demonstração seguiremos as notações estabelecidas na condição $\left(H_{1}\right)$. Primeiramente, observe que 1.6$)$ implica em

$$
I\left(u_{n}\right)-\frac{1}{\nu} I^{\prime}\left(u_{n}\right)\left(\theta \zeta u_{n}\right) \leq c+o_{n}(1)+o_{n}(1)\left\|u_{n}\right\|
$$

Por outro lado,

$$
\begin{aligned}
I\left(u_{n}\right) & -\frac{1}{\nu} I^{\prime}\left(u_{n}\right)\left(\theta \zeta u_{n}\right)=\frac{1}{N}|| u_{n} \|^{N}-\int_{\Omega} a(x) G\left(u_{n}\right) d x \\
& -\frac{\theta}{\nu} \int_{\Omega}\left(\left|\nabla u_{n}\right|^{N-2} \nabla u_{n} \nabla\left(\zeta u_{n}\right)+\left|u_{n}\right|^{N-2} u_{n}^{2} \zeta\right) d x+\frac{\theta}{\nu} \int_{\Omega} a(x) g\left(u_{n}\right) \zeta u_{n} d x \\
& \geq \frac{1}{N}|| u_{n} \|^{N}-\frac{\theta}{\nu} \int_{\Omega}\left|\nabla u_{n}\right|^{N} \zeta d x-\frac{\theta}{\nu} \int_{\Omega}\left|u_{n}\right|^{N} \zeta d x-\frac{\theta}{\nu} \int_{\Omega}\left|\nabla u_{n}\right|^{N-1}|\nabla \zeta|\left|u_{n}\right| d x \\
& +\frac{\theta}{\nu} \int_{\Omega^{+}} a(x) g\left(u_{n}\right) u_{n} \zeta d x-\int_{\Omega^{+}} a(x) G\left(u_{n}\right) \zeta d x,
\end{aligned}
$$

ou seja,

$$
\begin{aligned}
I\left(u_{n}\right)-\frac{1}{\nu} I^{\prime}\left(u_{n}\right)\left(\theta \zeta u_{n}\right) & \geq\left.\left(\frac{1}{N}-\frac{\theta}{\nu}\right)|| u_{n}\right|^{N}-\frac{\theta M}{\nu} \int_{\Omega}\left|\nabla u_{n}\right|^{N-1}\left|u_{n}\right| d x \\
& +\int_{\Omega^{+}} a(x)\left(\frac{\theta}{\nu} g\left(u_{n}\right) u_{n}-G\left(u_{n}\right)\right) d x
\end{aligned}
$$

Usando a hipótese $\left(H_{1}\right)$ e a Desigualdade de Young (ver Apêndice), temos

$$
I\left(u_{n}\right)-\frac{1}{\nu} I^{\prime}\left(u_{n}\right)\left(\theta \zeta u_{n}\right) \geq\left(\frac{1}{N}-\frac{\theta}{\nu}\right)\left\|u_{n}\right\|^{N}-\frac{\theta M}{\nu}\left(\frac{N-1}{N}\right)\left\|u_{n}\right\|^{N},
$$

ou ainda,

$$
I\left(u_{n}\right)-\frac{1}{\nu} I^{\prime}\left(u_{n}\right)\left(\theta \zeta u_{n}\right) \geq \frac{1}{N}\left(1-\frac{\theta}{\nu}(N+(N-1) M)\right)\left\|u_{n}\right\|^{N} .
$$

Combinando (1.14) com 1.15), obtemos

$$
\frac{1}{N}\left(1-\frac{\theta}{\nu}(N+(N-1) M)\right)\left\|u_{n}\right\|^{N} \leq c+o_{n}(1)+o_{n}(1)\left\|u_{n}\right\|
$$

Uma vez que $\left(1-\frac{\theta}{\nu}(N+(N-1) M)\right)>0$, concluímos que $\left(u_{n}\right)$ é limitada. 
Uma vez que $\left(u_{n}\right)$ é limitada em $W_{0}^{1, N}(\Omega)$, podemos supor que existem $u \in W_{0}^{1, N}(\Omega)$ e uma subsequência de $\left(u_{n}\right)$, ainda denotada por $\left(u_{n}\right)$, satisfazendo

$$
\left\{\begin{array}{cl}
u_{n} \rightarrow u & \text { em } W_{0}^{1, N}(\Omega), \\
u_{n}(x) \rightarrow u(x) & \text { q. t. p. em } \Omega, \\
u_{n} \rightarrow u & \text { em } L_{l o c}^{q}(\Omega), \text { para } q \geq 1 .
\end{array}\right.
$$

Lema 1.7 Seja $\left(u_{n}\right)$ a sequência Palais-Smale satisfazendo (1.6). Então, existe uma constante $C>0$ tal que

$$
\int_{\Omega^{ \pm}} a^{ \pm}(x) g\left(u_{n}\right) u_{n} d x \leq C, \quad \forall n \in \mathbb{N}
$$

onde $a^{ \pm}(x)=\max \{ \pm a(x), 0\}$. Além disso, para alguma subsequência de $\left(u_{n}\right)$, os seguintes limites são válidos:

$$
a^{ \pm}(x) g\left(u_{n}\right) \rightarrow a^{ \pm}(x) g(u) \quad \text { em } \quad L_{l o c}^{1}(\Omega)
$$

e

$$
a^{ \pm}(x) G\left(u_{n}\right) \rightarrow a^{ \pm}(x) G(u) \text { em } L_{l o c}^{1}(\Omega)
$$

onde u é o limite fraco de $\left(u_{n}\right)$ em $W_{0}^{1, N}(\Omega)$.

Demonstração. Considere $\sigma>\nu \operatorname{com} \sigma \approx \nu$. Repetindo os mesmos argumentos usados na prova do Lema 1.6 , obtemos

$$
\left(\frac{\sigma}{\nu}-\frac{\theta}{\nu}\right) \int_{\Omega^{+}} a(x) g\left(u_{n}\right) u_{n} \leq c+o_{n}(1)+\epsilon_{n}\left\|u_{n}\right\|+C_{1}\left\|u_{n}\right\|^{N},
$$

para alguma constante $C_{1}>0$. Sendo $\left(u_{n}\right)$ limitada em $W_{0}^{1, N}(\Omega)$, existe uma constante positiva $C$ tal que

$$
\int_{\Omega^{+}} a(x) g\left(u_{n}\right) u_{n} d x \leq C, \quad \forall n \in \mathbb{N}
$$

Uma vez que $I^{\prime}\left(u_{n}\right) u_{n}=o_{n}(1)$, temos 


$$
\int_{\Omega^{-}} a(x) g\left(u_{n}\right) u_{n} d x \leq \int_{\Omega} a(x) g\left(u_{n}\right) u_{n} d x=\left\|u_{n}\right\|^{N}+o_{n}(1),
$$

de onde segue que existe $C>0$ tal que

$$
\int_{\Omega^{-}} a(x) g\left(u_{n}\right) u_{n} d x \leq C, \quad \forall n \in \mathbb{N} .
$$

Portanto, a desigualdade (1.17) está justificada.

Para provar (1.18), observe inicialmente que

$$
\lim _{|t| \rightarrow+\infty} \frac{|g(t)|}{|g(t) t|}=0
$$

assim, dado $\epsilon>0$, existe $R_{\epsilon}>0$ tal que

$$
|g(t)| \leq \epsilon g(t) t, \quad \forall t \geq R_{\epsilon}
$$

Deste modo,

$$
\left|a^{+}(x) g(t)\right| \leq \epsilon a^{+}(x) g(t) t, \quad \forall x \in \Omega \text { e } \forall t \geq R_{\epsilon},
$$

consequetemente,

$$
\int_{\left[u_{n} \geq R_{\epsilon}\right] \cap \Omega^{+}} a(x)\left|g\left(u_{n}\right)\right| d x \leq \epsilon \int_{\left[u_{n} \geq R_{\epsilon}\right] \cap \Omega^{+}} a(x) g\left(u_{n}\right) u_{n} d x \leq \epsilon C
$$

e sem perda de generalidade, podemos assumir que

$$
\int_{\left[u \geq R_{\epsilon}\right] \cap \Omega^{+}} a(x)|g(u)| d x \leq \epsilon C .
$$

Agora, observe que

$$
\begin{aligned}
\int_{\Omega^{+}} a(x)\left|g\left(u_{n}\right)-g(u)\right| d x & \leq \int_{\left[u_{n}<R_{\epsilon}\right] \cap \Omega^{+}} a(x)\left|g\left(u_{n}\right)-g(u)\right| d x \\
& +\int_{\left[u_{n} \geq R_{\epsilon}\right] \cap \Omega^{+}} a(x)\left|g\left(u_{n}\right)\right| d x+\int_{\left[u_{n} \geq R_{\epsilon}\right] \cap \Omega^{+}} a(x)|g(u)| d x,
\end{aligned}
$$

$\log$,

$$
\begin{aligned}
\int_{\Omega^{+}} a(x)\left|g\left(u_{n}\right)-g(u)\right| d x & \leq \int_{\left[u_{n}<R_{\epsilon}\right] \cap \Omega^{+}} a(x)\left|g\left(u_{n}\right)-g(u)\right| d x \\
& +2 \epsilon C+\int_{S_{n}} a(x)|g(u)| d x
\end{aligned}
$$

onde $S_{n}=\left[u<R_{\epsilon}\right] \cap\left[u_{n} \geq R_{\epsilon}\right] \cap \Omega^{+}$. 
Uma vez que $\Omega^{+}$é limitado, segue do Teorema da Convergência Dominada de Lebesgue que ocorrem os seguintes limites

$$
\lim _{n \rightarrow+\infty} \int_{\left[u_{n}<R\right] \cap \Omega^{+}} a(x)\left|g\left(u_{n}\right)-g(u)\right| d x=0
$$

$\mathrm{e}$

$$
\lim _{n \rightarrow+\infty} \int_{S_{n}} a(x)|g(u)| d x=0,
$$

de onde podemos concluir que

$$
\lim _{n \rightarrow+\infty} \int_{\Omega^{+}} a(x) g\left(u_{n}\right) d x=\int_{\Omega^{+}} a(x) g(u) d x
$$

ou equivalentemente,

$$
\lim _{n \rightarrow+\infty} \int_{\Omega} a^{+}(x) g\left(u_{n}\right) d x=\int_{\Omega} a^{+}(x) g(u) d x
$$

e este último limite implica que

$$
a^{+}(x) g\left(u_{n}\right) \rightarrow a^{+}(x) g(u) \quad \text { em } \quad L_{l o c}^{1}(\Omega) .
$$

Com o mesmo tipo de argumento podemos provar que, para cada $r>0$

$$
\lim _{n \rightarrow+\infty} \int_{\Omega \cap B_{r}(0)} a^{-}(x) g\left(u_{n}\right) d x=\int_{\Omega \cap B_{r}(0)} a^{-}(x) g(u) d x,
$$

ou seja,

$$
a^{-}(x) g\left(u_{n}\right) \rightarrow a^{-}(x) g(u) \quad \text { em } \quad L^{1}(\Omega)
$$

Resta agora provar (1.19), para isto, observe que pela hipótese $\left(H_{2}\right)$, existem constantes positivas $C$ e $K_{0}$ tais que

$$
a^{ \pm} G\left(u_{n}\right) \leq C+K_{0} a^{ \pm}\left|g\left(u_{n}\right)\right|, \quad \forall n \in \mathbb{N} .
$$

Desta forma, os limites envolvendo as funções $a^{ \pm} g\left(u_{n}\right)$ combinados com o Teorema de Lebesgue implicam que

$$
a^{ \pm} G\left(u_{n}\right) \rightarrow a^{ \pm} G(u) \quad \text { em } \quad L_{l o c}^{1}(\Omega)
$$

completando a prova do lema.

Lema 1.8 Seja $\left(u_{n}\right)$ a sequência satisfazendo (1.6). Então, para alguma subsequência de $\left(u_{n}\right)$, ocorrem as seguintes convergências: 
(i) $\nabla u_{n}(x) \rightarrow \nabla u(x)$ q.t.p em $\Omega$;

(ii) $\int_{\Omega}\left|u_{n}\right|^{N-2} u_{n} v d x \rightarrow \int_{\Omega}|u|^{N-2} u v d x$, para cada $v \in W_{0}^{1, N}(\Omega)$;

(iii) $\int_{\Omega}\left|\nabla u_{n}\right|^{N-2} \nabla u_{n} \nabla v d x \rightarrow \int_{\Omega}|\nabla u|^{N-2} \nabla u \nabla v d x$, para cada $v \in W_{0}^{1, N}(\Omega)$,

onde u é o limite fraco de $\left(u_{n}\right)$ em $W_{0}^{1, N}(\Omega)$.

\section{Demonstração.}

Prova de $(i)$ : No que segue, para cada $L>0$, definimos as funções

$$
u_{n, L}(x)=\left\{\begin{array}{lll}
u_{n}(x), & \text { se } & \left|u_{n}(x)\right|<L \\
L, & \text { se } & u_{n}(x) \geq L \\
-L, & \text { se } & u_{n}(x) \leq-L
\end{array}\right.
$$

e

$$
u_{L}(x)=\left\{\begin{array}{lll}
u(x), & \text { se } & |u(x)|<L \\
L, & \text { se } & u(x) \geq L \\
-L, & \text { se } & u(x) \leq L .
\end{array}\right.
$$

Além disso, denotamos por $\eta(L)$, um termo que verifica $\eta(L) \rightarrow 0$ quando $L \rightarrow+\infty$.

Das definições de $u_{n, L}$ e $u_{L}$ é possível provar as seguintes propriedades:

a) $u_{L} \rightarrow u$ em $W_{0}^{1, N}(\Omega)$, quando $L \rightarrow+\infty$;

b) $u_{n, L} \rightarrow u_{L}$ em $W_{0}^{1, N}(\Omega)$, quando $n \rightarrow+\infty$;

c) para cada $B \subset \Omega$ limitado, temos que $|B \cap[|u| \geq L]| \rightarrow 0$ quando $L \rightarrow+\infty$,

onde $|A|$ denota a medida de Lebesgue do conjunto $A$;

d) para cada função não negativa $\Phi \in C_{0}^{\infty}(\Omega)$, vale

$$
\left|\int_{\Omega}\left\langle\left|\nabla u_{n}\right|^{N-2} \nabla u_{n}-|\nabla u|^{N-2} \nabla u, \nabla u_{L}-\nabla u\right\rangle \Phi d x\right| \leq C \eta(L), \quad \forall n \in \mathbb{N} .
$$


Denotamos por $P_{n}(x)$ e $P_{n, L}(x)$ as seguintes funções.

$$
\begin{gathered}
P_{n}=\left\langle\left|\nabla u_{n}\right|^{N-2} \nabla u_{n}-|\nabla u|^{N-2} \nabla u, \nabla u_{n}-\nabla u\right\rangle \Phi, \\
P_{n, L}=\left\langle\left|\nabla u_{n}\right|^{N-2} \nabla u_{n}-|\nabla u|^{N-2} \nabla u, \nabla u_{n, L}-\nabla u\right\rangle \Phi
\end{gathered}
$$

e

$$
A_{n, L}=\left\langle\left|\nabla u_{n}\right|^{N-2} \nabla u_{n}-|\nabla u|^{N-2} \nabla u, \nabla u_{n, L}-\nabla u_{L}\right\rangle \Phi .
$$

Por um cálculo direto, podemos deduzir que

$$
\limsup _{n \rightarrow+\infty} \int_{\Omega} P_{n, L} d x \leq \eta(L)+\limsup _{n \rightarrow+\infty} \int_{\Omega} A_{n, L} d x .
$$

Por outro lado, uma vez que $I^{\prime}\left(u_{n}\right)\left(\Phi u_{n, L}\right)=o_{n}(1)$ e $I^{\prime}\left(u_{n}\right)\left(\Phi u_{L}\right)=o_{n}(1)$, uniformemente em $L$, obtemos

$$
\begin{aligned}
\int_{\Omega} A_{n, L} d x & =\int_{\Omega} a(x)\left(u_{n, L}-u_{L}\right) g\left(u_{n}\right) \Phi d x+\int_{\Omega}\left(u_{L}-u_{n, L}\right)\left|\nabla u_{n}\right|^{N-2} \nabla u_{n} \nabla \Phi d x \\
& +\int_{\Omega}\left(u_{L}-u_{n, L}\right)\left|u_{n}\right|^{N-2} u_{n} \Phi d x+o_{n}(1) .
\end{aligned}
$$

O Teorema de Lebesgue juntamente com o Lema 1.7 implica que,

$$
\begin{gathered}
\lim _{n \rightarrow+\infty} \int_{\Omega} a(x)\left(u_{n, L}-u_{L}\right) g\left(u_{n}\right) \Phi d x=0, \\
\lim _{n \rightarrow+\infty} \int_{\Omega}\left(u_{L}-u_{n, L}\right)\left|\nabla u_{n}\right|^{N-2} \nabla u_{n} \nabla \Phi d x=0
\end{gathered}
$$

e

$$
\lim _{n \rightarrow+\infty} \int_{\Omega}\left(u_{L}-u_{n, L}\right)\left|u_{n}\right|^{N-2} u_{n} \Phi d x=0
$$

Assim,

$$
\lim _{n \rightarrow+\infty} \int_{\Omega} A_{n, L} d x=0
$$

De 1.20$)$ e 1.21$)$

$$
\limsup _{n \rightarrow+\infty} \int_{\Omega} P_{n, L} d x \leq \eta(L)
$$

Escolhendo $\beta \in(0,1)$ e fixando $B=\operatorname{supp} \Phi$, temos

$$
\int_{B}\left|P_{n}\right|^{\beta} d x \leq C \int_{B}\left|P_{n, L}\right|^{\beta} d x+C \int_{B}\left|\left\langle\left|\nabla u_{n}\right|^{N-2} \nabla u_{n}-\left|\nabla u_{n, L}\right|^{N-2} \nabla u_{n, L}, \nabla u_{n}-\nabla u_{n, L}\right\rangle\right|^{\beta} \Phi^{\beta} d x .
$$

Aplicando a Desigualdade de Hölder, encontramos $C>0$ tal que $\int_{B}\left|\left\langle\left|\nabla u_{n}\right|^{N-2} \nabla u_{n}-\left|\nabla u_{n, L}\right|^{N-2} \nabla u_{n, L}, \nabla u_{n}-\nabla u_{n, L}\right\rangle\right|^{\beta} d x \leq C\left|B \cap\left[\left|u_{n}\right|>L\right]\right|^{1-\beta}, \quad \forall n \in \mathbb{N}$. 
Por outro lado,

$$
\left|B \cap\left[\left|u_{n}\right|>L\right]\right| \leq\left|B \cap\left[\left|u_{n}\right|>L\right] \cap[|u|<L]\right|+\left|B \cap\left[\left|u_{n}\right|>L\right] \cap[|u| \geq L]\right|,
$$

isto é,

$$
\left|B \cap\left[\left|u_{n}\right|>L\right]\right| \leq\left|B \cap\left[\left|u_{n}\right|>L\right] \cap[|u|<L]\right|+|B \cap[|u| \geq L]| .
$$

Uma vez que

$$
\lim _{n \rightarrow+\infty}\left|B \cap\left[\left|u_{n}\right|>L\right] \cap[|u|<L]\right|=0 \quad \text { e }|B \cap[|u| \geq L]|=\eta(L),
$$

podemos concluir que

$$
\limsup _{n \rightarrow+\infty}\left|B \cap\left[\left|u_{n}\right|>L\right]\right| \leq \eta(L),
$$

de onde segue que

$$
\limsup _{n \rightarrow+\infty} \int_{B}\left|\left\langle\left|\nabla u_{n}\right|^{N-2} \nabla u_{n}-\left|\nabla u_{n, L}\right|^{N-2} \nabla u_{n, L}, \nabla u_{n}-\nabla u_{n, L}\right\rangle\right|^{\beta} d x \leq(\eta(L))^{1-\beta} .
$$

Desta forma,

$$
\limsup _{n \rightarrow+\infty} \int_{B} P_{n}^{\beta} d x \leq C \eta(L)+C \int_{B}\left|P_{n, L}\right|^{\beta} d x .
$$

Da Desigualdade de Hölder,

$$
\int_{B}\left|P_{n, L}\right|^{\beta} d x \leq \eta(L)\left(\int_{B \cap\left[\left|u_{n}\right| \geq L\right]}\left|P_{n, L}\right| d x\right)^{\beta}+C\left(\int_{B \cap\left[\left|u_{n}\right|<L\right]} P_{n, L} d x\right)^{\beta}
$$

$\log \mathrm{e}$

$$
\int_{B}\left|P_{n, L}\right|^{\beta} d x \leq C \eta(L)+C\left(\int_{B \cap\left[\left|u_{n}\right|<L\right]} P_{n, L} d x\right)^{\beta} .
$$

A desigualdade

$$
\int_{B \cap\left[\left|u_{n}\right|<L\right]} P_{n, L} d x \leq \int_{B} P_{n, L} d x+\int_{B \cap\left[\left|u_{n}\right| \geq L\right]}\left|P_{n, L}\right| d x
$$

combinada com

$$
\limsup _{n \rightarrow+\infty} \int_{B \cap\left[\left|u_{n}\right| \geq L\right]}\left|P_{n, L}\right| d x \leq \eta(L) \text { e } \quad \limsup _{n \rightarrow+\infty} \int_{B} P_{n, L} d x \leq \eta(L),
$$

implica que

$$
\limsup _{n \rightarrow+\infty} \int_{B \cap\left[u_{n}<L\right]} P_{n, L} d x \leq \eta(L),
$$

assim,

$$
\limsup _{n \rightarrow+\infty} \int_{B} P_{n}^{\beta} d x \leq \eta(L), \quad \forall L .
$$


Uma vez que $\eta(L) \rightarrow 0$ quando $L \rightarrow+\infty$, segue da última desigualdade que

$$
\limsup _{n \rightarrow+\infty} \int_{B} P_{n}^{\beta} d x=0
$$

Recordando que

$$
P_{n}^{\beta} \geq\left|\nabla u_{n}-\nabla u\right|^{\beta N} \Phi^{\beta} \geq 0,
$$

concluímos que

$$
\lim _{n \rightarrow+\infty} \int_{\Omega}\left|\nabla u_{n}-\nabla u\right|^{\beta N} \Phi^{\beta} d x=0 .
$$

Sendo $\Phi$ arbitrária, segue do último limite que, para alguma subsequência,

$$
\nabla u_{n}(x) \rightarrow \nabla u(x) \text { q.t.p. em } \Omega,
$$

finalizando a prova de $(i)$.

Prova de $(i i)$ : Para verificar $(i i)$ basta notar que, sendo $\left(\left|u_{n}\right|^{N-2} u_{n}\right)$ limitada em $L^{\frac{N}{N-1}}(\Omega)$ e $\left|u_{n}\right|^{N-2} u_{n} \rightarrow|u|^{N-2} u$ q. t. p. em $\Omega$, podemos aplicar um Lema de Brezis-Lieb (ver Apêndice) para concluir que

$$
\left|u_{n}\right|^{N-2} u_{n} \rightarrow|u|^{N-2} u \text { em } L^{\frac{N}{N-1}}(\Omega)
$$

Assim,

$$
\int_{\Omega}\left|u_{n}\right|^{N-2} u_{n} v d x \rightarrow \int_{\Omega}|u|^{N-2} u v d x, \quad \forall v \in W_{0}^{1, N}(\Omega)
$$

como queríamos provar.

Prova de $(i i i)$ : Do item $(i)$, segue que

$$
\left|\nabla u_{n}\right|^{N-2} \nabla u_{n} \rightarrow|\nabla u|^{N-2} \nabla u \text { q.t.p. em } \Omega \text {. }
$$

Uma vez que $\left(\left|\nabla u_{n}\right|^{N-2} \nabla u_{n}\right)$ é limitada em $L^{\frac{N}{N-1}}(\Omega)$, temos

$$
\left|\nabla u_{n}\right|^{N-2} \nabla u_{n} \rightarrow|\nabla u|^{N-2} \nabla u \text { em } L^{\frac{N}{N-1}}(\Omega) .
$$

Portanto, pelo Lema de Brezis-Lieb

$$
\int_{\Omega}\left|\nabla u_{n}\right|^{N-2} \nabla u_{n} \nabla v d x \rightarrow \int_{\Omega}|\nabla u|^{N-2} \nabla u \nabla v d x
$$

provando (iii). 


\subsection{Existência de solução}

Estamos prontos para provar o resultado de existência de solução para o problema $\left(P_{1}\right)$.

\section{Demonstração do Teorema 1}

Começaremos mostrando que o limite fraco $u$, da sequência Palais-Smale $\left(u_{n}\right)$, é uma solução de $\left(P_{1}\right)$. De fato, aplicando os Lemas 1.7 e 1.8 , para cada $\varphi \in C_{0}^{\infty}(\Omega)$, temos

$$
\begin{aligned}
o_{n}(1) & =I^{\prime}\left(u_{n}\right) \varphi=\int_{\Omega}\left(\left|\nabla u_{n}\right|^{N-2} \nabla u_{n} \nabla \varphi+\left|u_{n}\right|^{N-2} u_{n} \varphi\right) d x-\int_{\Omega} a(x) g\left(u_{n}\right) \varphi d x \\
& =\int_{\Omega}\left(|\nabla u|^{N-2} \nabla u \nabla \varphi+|u|^{N-2} u \varphi\right) d x-\int_{\Omega} a(x) g(u) \varphi d x+o_{n}(1) \\
& =I^{\prime}(u) \varphi+o_{n}(1),
\end{aligned}
$$

ou seja, $I^{\prime}(u) \varphi=0$. Pela densidade de $C_{0}^{\infty}(\Omega)$ em $W_{0}^{1, N}(\Omega)$, obtemos que $I^{\prime}(u) v=0$, para toda $v \in W_{0}^{1, N}(\Omega)$. Portanto, $u$ é uma solução para o problema $\left(P_{1}\right)$.

Resta agora provar que $u$ é não trivial. Para isto, suponha por contradição que $u \equiv 0$ e observe que

$$
\begin{aligned}
c+o_{n}(1) & =I\left(u_{n}\right)=\frac{1}{N}\left\|u_{n}\right\|^{N}-\int_{\Omega} a(x) G\left(u_{n}\right) d x \\
& =\frac{1}{N}\left\|u_{n}\right\|^{N}-\int_{\Omega^{-}} a(x) G\left(u_{n}\right) d x-\int_{\Omega^{+}} a(x) G\left(u_{n}\right) d x \\
& \geq \frac{1}{N}\left\|u_{n}\right\|^{N}-\int_{\Omega^{+}} a(x) G\left(u_{n}\right) d x .
\end{aligned}
$$

Pelo Lema 1.7 ,

$$
\int_{\Omega^{+}} a(x) G\left(u_{n}\right) d x \rightarrow 0
$$

assim,

$$
c+o_{n}(1) \geq \frac{1}{N}\left\|u_{n}\right\|^{N}-o_{n}(1)
$$

Uusando o Lema 1.5, obtemos

$$
\limsup _{n \rightarrow \infty}\left\|u_{n}\right\|^{N} \leq N c<\left(\frac{\alpha_{N}}{\alpha_{0}}\right)^{N-1} .
$$

Considerando $C_{0}=\sup _{\Omega^{+}} a(x)$, segue de $\left(H_{0}\right)$ e $\left(H_{c}\right)$ que, para cada $x \in \Omega^{+}$,

$$
\left|a(x) g\left(u_{n}\right) u_{n}\right| \leq C_{0}\left[\left|u_{n}\right|^{N}+C\left|u_{n}\right|\left(e^{\alpha\left|u_{n}\right|^{\frac{N}{N-1}}}-S_{N-2}\left(\alpha, u_{n}\right)\right)\right] .
$$

Sejam $\alpha$ e $t$ dados na prova do Lema 1.3 e defina

$$
Q_{n}:=\left[e^{\alpha\left|u_{n}\right|^{\frac{N}{N-1}}}-S_{N-2}\left(\alpha, u_{n}\right)\right] .
$$


Do Lema 1.3. $Q_{n} \in L^{t}\left(\Omega^{+}\right)$, para cada $n \geq n_{0}$ e $\left(Q_{n}\right)$ é limitada em $L^{t}\left(\Omega^{+}\right)$. Além disso, $Q_{n}(x) \rightarrow 0$ q.t.p. em $\Omega^{+}$. Logo, aplicando um Lema de Brezis-Lieb,

$$
Q_{n} \rightarrow 0 \quad \text { em } \quad L^{t}\left(\Omega^{+}\right)
$$

Uma vez que $u_{n} \rightarrow 0$ em $L_{l o c}^{q}(\Omega)$, para cada $q \geq 1$, em particular,

$$
u_{n} \rightarrow 0 \text { em } L^{t^{\prime}}\left(\Omega^{+}\right)
$$

onde $t^{\prime}=t /(t-1)$. Portanto, de 1.23$)-1.24$,

$$
\int_{\Omega^{+}}\left|u_{n}\right| Q_{n} d x \rightarrow 0
$$

Combinando 1.22 com 1.25, segue do Teorema da Convergência Dominada de Lebesgue que

$$
\int_{\Omega^{+}} a(x) g\left(u_{n}\right) u_{n} d x \rightarrow 0
$$

Agora, usando que $I^{\prime}\left(u_{n}\right) u_{n}=o_{n}(1)$, obtemos

$$
\left\|u_{n}\right\|^{N} \leq \int_{\Omega^{+}} a(x) g\left(u_{n}\right) u_{n} d x+o_{n}(1)
$$

mostrando que

$$
\lim _{n \rightarrow \infty}\left\|u_{n}\right\|^{N}=0
$$

o que implica que $u_{n} \rightarrow 0$ em $W_{0}^{1, N}(\Omega)$. Consequentemente,

$$
c=\lim _{n \rightarrow \infty} I\left(u_{n}\right)=0
$$

o que contradiz o fato que $c>0$. Portanto, $u \in W_{0}^{1, N}(\Omega)$ é uma solução do problema $\left(P_{1}\right)$, como queríamos provar. 


\title{
CAPÍTULO 2
}

MULTIPLICIDADE DE SOLUÇÕES VIA MINIMIZAÇÃO

\author{
LOCAL E PASSO DA MONTANHA
}

Considerando o problema $(P)$, apresentado na Introdução, vamos trabalhar com dois casos. Primeiramente, estudamos o caso em que $f(x, u)=-|u|^{N-2} u+\lambda|u|^{q-2} u+g(u)$ e $\Omega$ é limitado. Em seguida, consideramos $f(x, u)=\lambda h(x)|u|^{q-2} u+g(u)$ e $\Omega=\mathbb{R}^{N}$. Analisamos as questões de existência e multiplicidade de soluções, utilizando Teorema do Passo da Montanha, Princípio Variacional de Ekeland e resultados envolvendo Teoria do Gênero. Dividimos este capítulo em duas seções para estudar os casos acima.

\subsection{Soluções para uma classe de equações em domínio}

\section{limitado}

Nesta seção, vamos considerar o problema

$$
\left\{\begin{array}{l}
-\Delta_{N} u=\lambda|u|^{q-2} u+g(u), \quad x \in \Omega, \\
u=0, \quad x \in \partial \Omega
\end{array}\right.
$$

onde $\Omega \subset \mathbb{R}^{N}$ é um domínio limitado com fronteira suave, $N \geq 2,1<q<N$ e $\lambda>0$. 
Admitimos que a função $g$ satisfaz as seguintes hipóteses:

$\left(\mathbf{H}_{\mathbf{0}}\right) g: \mathbb{R} \rightarrow \mathbb{R}$ é uma função contínua tal que

$$
g(s)=o\left(|s|^{N-1}\right), \text { quando } s \rightarrow 0
$$

$\left(\mathbf{H}_{\mathbf{c}}\right)$ existe $\alpha_{0}>0$ tal que

$$
\lim _{|s| \rightarrow \infty} \frac{|g(s)|}{e^{\alpha|s|^{\frac{N}{N-1}}}}=\left\{\begin{array}{lll}
0, & \text { se } & \alpha>\alpha_{0} \\
+\infty, & \text { se } & \alpha<\alpha_{0}
\end{array}\right.
$$

$\left(\mathbf{H}_{4}\right)$ existe $\nu>N$ tal que

$$
0<\nu G(s) \leq g(s) s, \quad \forall|s|>0
$$

onde $G(s)=\int_{0}^{s} g(t) d t$

$\left(\mathbf{H}_{\mathbf{5}}\right)$ existem $p>N$ e uma constante $C_{p}>0$ tais que

$$
g(s) \geq C_{p} s^{p-1}, \quad \forall s \geq 0
$$

Vamos assumir que

$$
C_{p}>S_{p}^{p}\left\{\frac{p(\nu-N)}{\nu(p-N)}\left(\frac{\alpha_{N}}{\alpha_{0}}\right)^{N-1}\right\}^{\frac{N-p}{N}}
$$

com

$$
S_{p}:=\inf _{v \in W_{0}^{1, N}(\Omega) \backslash\{0\}} \frac{\|v\|_{W_{0}^{1, N}(\Omega)}}{|v|_{L^{p}(\Omega)}} .
$$

Nas duas próximas subseções vamos supor que $g(s)=0$, para $s<0$, pois desejamos obter soluções positivas. O primeiro passo será encontrar uma solução de $\left(P_{2}\right)$ com energia positiva. Para isto, exploramos idéias similares as desenvolvidas no Capítulo 1, onde novamente o Teorema do Passo da Montanha e a Desigualdade de Trudinger-Moser são as principais ferramentas. Em seguida, usando o Princípio Variacional de Ekeland, provamos a existência de uma outra solução, com energia negativa. Iniciamos apresentando a seguinte versão da Desigualdade de Trudinger-Moser (ver Trudinger [77], Moser [60]). 


\section{Lema 2.1 (Desigualdade de Trudinger-Moser para domínios limitados)}

Seja $\Omega$ um dominio limitado em $\mathbb{R}^{N}, N \geq 2$. Então, para qualquer $u \in W_{0}^{1, N}(\Omega)$,

$$
\int_{\Omega} e^{\alpha|u|^{N-1}} d x<\infty, \quad \forall \alpha>0
$$

Além disso, se $|\nabla u|_{N}^{N} \leq 1$, e $\alpha \leq \alpha_{N}=N \omega_{N-1}^{\frac{1}{N-1}}$, então existe uma constante positiva $C=C(N,|\Omega|)$ tal que

$$
\int_{\Omega} e^{\alpha|u|^{N-1}} d x \leq C
$$

onde $\omega_{N-1}$ é a medida $(N-1)$-dimensional da $(N-1)$-esfera.

No espaço de Sobolev $W_{0}^{1, N}(\Omega)$ consideramos a norma

$$
\|u\|=\left(\int_{\Omega}|\nabla u|^{N} d x\right)^{1 / N}, \quad u \in W_{0}^{1, N}(\Omega) .
$$

Associamos ao problema $\left(P_{2}\right)$ o funcional energia $I_{\lambda}: W_{0}^{1, N}(\Omega) \rightarrow \mathbb{R}$ definido por

$$
I_{\lambda}(u)=\frac{1}{N} \int_{\Omega}|\nabla u|^{N} d x-\frac{\lambda}{q} \int_{\Omega} u_{+}^{q} d x-\int_{\Omega} G(u) d x .
$$

O funcional $I_{\lambda}$ pertence a $C^{1}\left(W_{0}^{1, N}(\Omega), \mathbb{R}\right)$ e a derivada de $I_{\lambda}$ é dada por

$$
I_{\lambda}^{\prime}(u) v=\int_{\Omega}|\nabla u|^{N-2} \nabla u \nabla v d x-\lambda \int_{\Omega} u_{+}^{q-1} v d x-\int_{\Omega} g(u) v d x, \quad u, v \in W_{0}^{1, N}(\Omega) .
$$

\subsubsection{Primeira solução}

Lema 2.2 Existe $\lambda_{1}>0$ tal que, para cada $0<\lambda<\lambda_{1}$, o funcional $I_{\lambda}$ satisfaz as condições:

(i) Existem $\beta, \rho>0$ tais que

$$
I_{\lambda}(u) \geq \beta, \quad \text { para }\|u\|=\rho .
$$

(ii) Existe e $\in W_{0}^{1, N}(\Omega)$ tal que $\|e\|>\rho$ e $I_{\lambda}(e)<0$. 
Demonstração. Por $\left(H_{0}\right)$ e $\left(H_{c}\right)$, dados $\epsilon>0$ e $s \geq 1$, existe $C_{\epsilon}=C(\epsilon, s)>0$ tal que, para cada $\alpha>\alpha_{0}$

$$
|G(t)| \leq \frac{\epsilon}{N}|t|^{N}+C|t|^{s} e^{\alpha|t|^{\frac{N}{N-1}}}, \quad \forall t \in \mathbb{R} .
$$

Vamos fixar $R>0$ verificando $\alpha R^{\frac{N}{N-1}}<\alpha_{N}$ e escolher $t_{1}>1\left(t_{1} \approx 1\right)$ tal que $t_{1} \alpha R^{\frac{N}{N-1}}<\alpha_{N}$ e $t_{2}>1$ tal que $\frac{1}{t_{1}}+\frac{1}{t_{2}}=1$. Segue da Desigualdade de Hölder que, para cada $u \in W_{0}^{1, N}(\Omega)$ com $\|u\| \leq R$

$$
I_{\lambda}(u) \geq \frac{1}{N} \int_{\Omega}|\nabla u|^{N} d x-\frac{\lambda}{q} \int_{\Omega} u_{+}^{q} d x-\frac{\epsilon}{N}|u|_{N}^{N}-C_{\epsilon}|u|_{s t_{2}}^{s}\left\{\int_{\Omega} e^{t_{1} \alpha R^{N-1}\left(\frac{|u|}{\|u\|}\right)^{N-1}} d x\right\}^{\frac{1}{t_{1}}} .
$$

Combinando a Desigualdade de Trudinger-Moser (Lema 2.1) com a imersão de Sobolev

$$
W_{0}^{1, N}(\Omega) \hookrightarrow L^{t}(\Omega), 1 \leq t<\infty
$$

deduzimos

$$
I_{\lambda}(u) \geq \frac{1}{N}\|u\|^{N}-\frac{\lambda}{q} C_{1}\|u\|^{q}-\frac{\epsilon}{N} C_{2}\|u\|^{N}-C_{\epsilon}\|u\|^{s} .
$$

Fixando $\epsilon=\frac{1}{2 C_{2}}$, escrevemos

$$
I_{\lambda}(u) \geq \frac{1}{2 N}\|u\|^{N}-\frac{C_{1}}{q} \lambda\|u\|^{q}-C_{3}\|u\|^{s}, \quad \forall u \in W_{0}^{1, N}(\Omega),\|u\| \leq R .
$$

Escolhendo $s>N$, podemos fixar $0<\rho<R$ suficientemente pequeno verificando

$$
\beta:=\frac{1}{4 N} \rho^{N}-C_{3} \rho^{s}>0 .
$$

Observe que se $\lambda<\lambda_{1}:=\frac{q}{4 N C_{1}} \rho^{N-q}$, então

$$
I_{\lambda}(u)>\frac{1}{2 N} \rho^{N}-\frac{C_{1}}{q} \frac{q}{4 N C_{1}} \rho^{N-q} \rho^{q}-C_{3} \rho^{s}=\frac{1}{4 N} \rho^{N}-C_{3} \rho^{s},
$$

para $\|u\|=\rho$. Portanto,

$$
I_{\lambda}(u)>\beta>0, \quad\|u\|=\rho,
$$

assim, $(i)$ está provado.

Fixe $v \in W_{0}^{1, N}(\Omega) \backslash\{0\}$. Usando a hipótese $\left(H_{4}\right)$, temos

$$
I_{\lambda}(t v) \leq \frac{t^{N}}{N}\|v\|^{N}-\frac{t^{q}}{q} \lambda|v|_{q}^{q}-C t^{\nu} \int_{\Omega}|v|^{\nu} d x+D
$$

Uma vez que $\nu>N$, temos que $I_{\lambda}(t v) \rightarrow-\infty$ quando $t \rightarrow+\infty$. Fixando $e=t v$ com $t>0$ suficientemente grande, concluímos $(i i)$. 
Aplicando o Teorema do Passo da Montanha sem a condição Palais-Smale (ver Apêndice, Teorema A.6), garantimos a existência de uma sequência Palais-Smale no nível $c$, isto é, existe uma sequência $\left(u_{n}\right) \subset W_{0}^{1, N}(\Omega)$ satisfazendo

$$
I_{\lambda}\left(u_{n}\right) \rightarrow c \quad \text { e } \quad I_{\lambda}^{\prime}\left(u_{n}\right) \rightarrow 0, \quad \text { quando } n \rightarrow+\infty
$$

onde

$$
c=\inf _{\gamma \in \Gamma} \max _{t \in[0,1]} I_{\lambda}(\gamma(t)),
$$

com

$$
\Gamma=\left\{\gamma \in C\left([0,1], W_{0}^{1, N}(\Omega)\right) ; \gamma(0)=0 \text { e } \gamma(1)=e\right\}
$$

onde $e$ é dado pelo Lema 2.2 .

Observação 2.1 Sem perda de generalidade, podemos supor que $u_{n} \geq 0, \forall n \in \mathbb{N}$. Pois, com as mesmas idéias encontradas em Alves [8], podemos verificar que a sequência $\left(\left(u_{n}\right)_{+}\right)$ também é uma sequência $(P . S)_{c}$ para $I_{\lambda}$.

Lema 2.3 O nível c do Passo da Montanha satisfaz

$$
c<\left(\frac{1}{N}-\frac{1}{\nu}\right)\left(\frac{\alpha_{N}}{\alpha_{0}}\right)^{N-1} .
$$

Demonstração. Seja $\varphi \in W_{0}^{1, N}(\Omega) \backslash\{0\}$ uma função satisfazendo

$$
\frac{\|\varphi\|}{|\varphi|_{p}}=S_{p}:=\inf _{v \in W_{0}^{1, N}(\Omega) \backslash\{0\}} \frac{\|v\|}{|v|_{p}} .
$$

De acordo com a prova do Lema 2.2 podemos considerar o caminho $\gamma(t)=t t_{0} \varphi$, onde $t_{0}>0$ é suficientemente grande tal que $e=t_{0} \varphi$. Logo, pela definição de $c$,

$$
c \leq \max _{t \geq 0} I_{\lambda}(t \varphi)
$$

isto é,

$$
c \leq \max _{t \geq 0}\left\{\frac{t^{N}}{N}\|\varphi\|^{N}-\int_{\Omega} G(t \varphi) d x\right\} .
$$

Usando $\left(H_{5}\right)$, ficamos com

$$
c \leq \max _{t \geq 0}\left\{\frac{t^{N}}{N}\|\varphi\|^{N}-\frac{C_{p}}{p} t^{p} \int_{\Omega}|\varphi|^{p} d x\right\},
$$


ou ainda,

$$
\frac{c}{|\varphi|_{p}^{N}} \leq \max _{t \geq 0}\left\{\frac{t^{N}}{N} S_{p}^{N}-\frac{C_{p}}{p} t^{p}|\varphi|_{p}^{p-N}\right\}
$$

Uma vez que a função

$$
h(t)=\frac{t^{N}}{N} S_{p}^{N}-\frac{C_{p}}{p} t^{p}|\varphi|_{p}^{p-N}
$$

atinge máximo absoluto em

$$
t_{0}=C_{p}^{\frac{1}{N-p}}|\varphi|_{p}^{-1} S_{p}^{\frac{N}{p-N}}
$$

conclui-se que

$$
c \leq\left(\frac{1}{N}-\frac{1}{p}\right) C_{p}^{\frac{N}{N-p}} S_{p}^{\frac{p N}{p-N}} .
$$

Mas, por hipótese

$$
C_{p}>S_{p}^{p}\left\{\frac{p(\nu-N)}{\nu(p-N)}\left(\frac{\alpha_{N}}{\alpha_{0}}\right)^{N-1}\right\}^{\frac{N-p}{N}}
$$

Portanto,

$$
c<\left(\frac{1}{N}-\frac{1}{\nu}\right)\left(\frac{\alpha_{N}}{\alpha_{0}}\right)^{N-1}
$$

Lema 2.4 Existe $\lambda_{2}>0$ tal que, para $\lambda<\lambda_{2}$, a sequência Palais-Smale $\left(u_{n}\right)$ associada ao funcional $I_{\lambda}$ verifica

$$
\limsup _{n \rightarrow \infty}\left\|u_{n}\right\|^{N}<\left(\frac{\alpha_{N}}{\alpha_{0}}\right)^{N-1}
$$

Demonstração. Observe que a sequência $\left(u_{n}\right)$ é limitada em $W_{0}^{1, N}(\Omega)$. De fato, por 2.3 )

$$
I_{\lambda}\left(u_{n}\right)-\frac{1}{\nu} I_{\lambda}^{\prime}\left(u_{n}\right)\left(u_{n}\right) \leq c+o_{n}(1)+o_{n}(1)\left\|u_{n}\right\| .
$$

Por outro lado,

$$
I_{\lambda}\left(u_{n}\right)-\frac{1}{\nu} I_{\lambda}^{\prime}\left(u_{n}\right)\left(u_{n}\right)=\left(\frac{1}{N}-\frac{1}{\nu}\right)\left\|u_{n}\right\|^{N}-\lambda\left(\frac{1}{q}-\frac{1}{\nu}\right)\left|u_{n}\right|_{q}^{q}-\int_{\Omega}\left(G\left(u_{n}\right)-\frac{1}{\nu} g\left(u_{n}\right) u_{n}\right) d x .
$$

Assim, por $\left(H_{4}\right)$

$$
I_{\lambda}\left(u_{n}\right)-\frac{1}{\nu} I_{\lambda}^{\prime}\left(u_{n}\right)\left(u_{n}\right) \geq\left(\frac{1}{N}-\frac{1}{\nu}\right)\left\|u_{n}\right\|^{N}-\lambda\left(\frac{1}{q}-\frac{1}{\nu}\right)\left|u_{n}\right|_{q}^{q} .
$$

Combinando (2.5) com 2.6), obtemos

$$
\left(\frac{1}{N}-\frac{1}{\nu}\right)\left\|u_{n}\right\|^{N} \leq \lambda\left(\frac{1}{q}-\frac{1}{\nu}\right)\left|u_{n}\right|_{q}^{q}+c+o_{n}(1)+o_{n}(1)\left\|u_{n}\right\| .
$$


Usando a imersão de Sobolev $W_{0}^{1, N}(\Omega) \hookrightarrow L^{q}(\Omega)$, segue que

$$
\left(\frac{1}{N}-\frac{1}{\nu}\right)\left\|u_{n}\right\|^{N} \leq \lambda\left(\frac{1}{q}-\frac{1}{\nu}\right) C\left\|u_{n}\right\|^{q}+c+o_{n}(1)+o_{n}(1)\left\|u_{n}\right\| .
$$

Uma vez que $q<N$, concluímos que $\left(u_{n}\right)$ é limitada em $W_{0}^{1, N}(\Omega)$.

Aplicando a Desigualdade de Hölder em (2.7), ficamos com

$$
\begin{aligned}
\left(\frac{1}{N}-\frac{1}{\nu}\right)\left\|u_{n}\right\|^{N} & \leq\left(\frac{1}{q}-\frac{1}{\nu}\right)\left(\int_{\Omega} \lambda^{\frac{N}{N-q}} d x\right)^{\frac{N-q}{N}}\left(\int_{\Omega}\left|u_{n}\right|^{N} d x\right)^{\frac{q}{N}}+c+o_{n}(1)+o_{n}(1)\left\|u_{n}\right\| \\
& \leq\left(\frac{1}{q}-\frac{1}{\nu}\right)\left\{C_{\epsilon} \int_{\Omega} \lambda^{\frac{N}{N-q}} d x+\epsilon \int_{\Omega}\left|u_{n}\right|^{N} d x\right\}+c+o_{n}(1)+o_{n}(1)\left\|u_{n}\right\|,
\end{aligned}
$$

onde na última estimativa, aplicamos a Desigualdade de Young (ver Apêndice). Usando a imersão de Sobolev $W_{0}^{1, N}(\Omega) \hookrightarrow L^{N}(\Omega)$, temos

$$
\left\{\left(\frac{1}{N}-\frac{1}{\nu}\right)-\epsilon\left(\frac{1}{q}-\frac{1}{\nu}\right) C\right\}\left\|u_{n}\right\|^{N} \leq\left(\frac{1}{q}-\frac{1}{\nu}\right) C_{\epsilon} \lambda^{\frac{N}{N-q}}|\Omega|+c+o_{n}(1)+o_{n}(1)\left\|u_{n}\right\| .
$$

Fixando $\epsilon=\frac{\left(\frac{1}{N}-\frac{1}{\nu}\right)}{2\left(\frac{1}{q}-\frac{1}{\nu}\right) C}>0$, obtemos

$$
\frac{1}{2}\left(\frac{1}{N}-\frac{1}{\nu}\right)\left\|u_{n}\right\|^{N} \leq C \lambda^{\frac{N}{N-q}}+c+o_{n}(1)+o_{n}(1)\left\|u_{n}\right\|
$$

ou ainda,

$$
\left\|u_{n}\right\|^{N} \leq C \lambda^{\frac{N}{N-q}}+\frac{c}{\frac{1}{2}\left(\frac{1}{N}-\frac{1}{\nu}\right)}+o_{n}(1)+o_{n}(1)\left\|u_{n}\right\|
$$

Portanto,

$$
\limsup _{n \rightarrow \infty}\left\|u_{n}\right\|^{N} \leq C \lambda^{\frac{N}{N-q}}+\frac{c}{\frac{1}{2}\left(\frac{1}{N}-\frac{1}{\nu}\right)},
$$

onde $C$ é uma constante positiva que depende apenas de $N,|\Omega|, q$ e $\nu$.

Como consequência do Lema 2.3 .

$$
\limsup _{n \rightarrow \infty}\left\|u_{n}\right\|^{N}<C \lambda^{\frac{N}{N-q}}+\frac{1}{2}\left(\frac{\alpha_{N}}{\alpha_{0}}\right)^{N-1} .
$$

Escolhendo $\lambda_{2}:=\left(\frac{1}{2 C}\left(\frac{\alpha_{N}}{\alpha_{0}}\right)^{N-1}\right)^{\frac{N-q}{N}}$, concluímos que

$$
\limsup _{n \rightarrow \infty}\left\|u_{n}\right\|^{N}<\left(\frac{\alpha_{N}}{\alpha_{0}}\right)^{N-1}
$$

para $\lambda<\lambda_{2}$, provando o lema.

O próximo lema é a versão do Lema 1.3 para domínios limitados. Novamente, será essencial para obter as convergências necessárias ao nosso estudo. 
Lema 2.5 Seja $\left(u_{n}\right)$ uma sequência satisfazendo (2.4). Então, existem $\alpha>\alpha_{0}, t>1 e$ $C>0$, independente de $n$, tais que

$$
\int_{\Omega} e^{t \alpha\left|u_{n}\right|^{N-1}} d x \leq C, \quad \forall n \geq n_{0}
$$

para algum $n_{0}$ suficientemente grande.

Demonstração. A demonstração segue com o mesmo raciocínio aplicado na prova do Lema 1.3. De fato, sejam $m>0$ e $n_{0} \in \mathbb{I}$ tais que

$$
\left\|u_{n}\right\|^{\frac{N}{N-1}}<m<\frac{\alpha_{N}}{\alpha_{0}}, \quad \forall n \geq n_{0} .
$$

Escolha $\alpha>\alpha_{0}$ e $t>1$ satisfazendo $\alpha m<\alpha_{N}$, e $t \alpha m<\alpha_{N}$. Pela Desigualdade de Trudinger-Moser (ver Lema 2.1), existe $C>0$ independente de $n$ tal que

$$
\int_{\Omega} e^{t \alpha\left|u_{n}\right|^{\frac{N}{N-1}}} \leq C \int_{\Omega} e^{t \alpha m\left(\frac{u_{n}}{\left\|u_{n}\right\|}\right)^{\frac{N}{N-1}}} \leq C, \quad \forall n \geq n_{0}
$$

Vimos no Lema 2.4 que a sequência $\left(u_{n}\right)$ dada em 2.3 é limitada em $W_{0}^{1, N}(\Omega)$, assim, podemos supor que existe $u_{1} \in W_{0}^{1, N}(\Omega)$ tal que, a menos de subsequência, $\left(u_{n}\right)$ satisfaz

$$
\left\{\begin{aligned}
u_{n} \rightarrow u_{1} & \text { em } W_{0}^{1, N}(\Omega), \\
u_{n}(x) \rightarrow u_{1}(x) & \text { q. t. p. em } \Omega \\
u_{n} \rightarrow u_{1} & \text { em } L^{q}(\Omega), \text { para } q \geq 1 .
\end{aligned}\right.
$$

Lema 2.6 Os seguintes limites são verdadeiros:

(i) $\int_{\Omega}\left(g\left(u_{n}\right) u_{n}-g\left(u_{n}\right) u_{1}\right) d x=o_{n}(1)$;

(ii) $\int_{\Omega}\left(\left|u_{n}\right|^{q}-\left|u_{n}\right|^{q-2} u_{n} u_{1}\right) d x=o_{n}(1)$;

(iii) $\nabla u_{n}(x) \rightarrow \nabla u_{1}(x)$ q.t.p. em $\Omega$;

(iv) $\int_{\Omega}\left(\left|\nabla u_{n}\right|^{N-2} \nabla u_{n} \nabla v-\left|\nabla u_{1}\right|^{N-2} \nabla u_{1} \nabla v\right) d x=o_{n}(1), \quad \forall v \in W_{0}^{1, N}(\Omega)$. 


\section{Demonstração.}

Prova de $(i)$ : Observe que

$$
\left|g\left(u_{n}\right) u_{n}-g\left(u_{n}\right) u_{1}\right| \leq\left|u_{n}\right|^{N-1}\left|u_{n}-u_{1}\right|+C\left|u_{n}-u_{1}\right| e^{\alpha\left|u_{n}\right|^{\frac{N}{N-1}}} .
$$

Considere $\alpha$ e $t$ dados pelo Lema 2.5. Para simplificar, denotamos

$$
Q_{n}:=e^{\alpha\left|u_{n}\right|^{\frac{N}{N-1}}} .
$$

Pelo Lema 2.5. $\left(Q_{n}\right)$ é uniformemente limitada em $L^{t}(\Omega)$. Logo, a Desigualdade de Hölder em (2.9) acarreta em

$$
\int_{\Omega}\left|g\left(u_{n}\right) u_{n}-g\left(u_{n}\right) u_{1}\right| d x \leq C|| u_{n}||^{N-1}\left|u_{n}-u_{1}\right|_{N}+C\left|u_{n}-u_{1}\right|_{t^{\prime}},
$$

onde $t^{\prime}$ é o expoente conjugado de $t$. A demonstração segue, usando que $u_{n} \rightarrow u_{1}$ em $L^{q}(\Omega)$ para $q \geq 1$.

Prova de $(i i)$ : Para justificar $(i i)$ basta notar que, sendo $\left(\left|u_{n}\right|^{q-1}\right)$ limitada em $L^{\frac{q}{q-1}}(\Omega)$, temos

$$
\left|\int_{\Omega}\left(\left|u_{n}\right|^{q}-\left|u_{n}\right|^{q-2} u_{n} u_{1}\right) d x\right| \leq \int_{\Omega}\left|u_{n}\right|^{q-1}\left|u_{n}-u_{1}\right| d x \leq C\left|u_{n}-u_{1}\right|_{q}=o_{n}(1) .
$$

Prova de $(i i i)$ e $(i v)$ : Segue de modo análogo a prova do Lema 1.8 .

Proposição 2.1 Suponha que $g$ satisfaz $\left(H_{0}\right),\left(H_{c}\right),\left(H_{4}\right)$ e $\left(H_{5}\right)$. Então, existe uma constante $\Lambda_{1}>0$ tal que, para $0<\lambda<\Lambda_{1}$, o problema $\left(P_{2}\right)$ possui uma solução $u_{1}$ verificando $I_{\lambda}\left(u_{1}\right)>0$.

Demonstração. Considere $u_{1}$ o limite fraco da sequência $\left(u_{n}\right)$ que verifica (2.3). Inicialmente, considere $\Lambda_{1}=\min \left\{\lambda_{1}, \lambda_{2}\right\}$, onde $\lambda_{1}$ e $\lambda_{2}$ são dados nos Lemas 2.2 e 2.4. respectivamente. Para provar a Proposição 2.1 basta mostrar que $u_{n} \rightarrow u_{1}$ em $W_{0}^{1, N}(\Omega)$. Com efeito, observe que

$$
\begin{aligned}
\int_{\Omega}\left|\nabla u_{n}\right|^{N} d x & =I_{\lambda}^{\prime}\left(u_{n}\right) u_{n}+\lambda \int_{\Omega}\left|u_{n}\right|^{q} d x+\int_{\Omega} g\left(u_{n}\right) u_{n} d x-I_{\lambda}^{\prime}\left(u_{n}\right) u_{1} \\
& +\int_{\Omega}\left|\nabla u_{n}\right|^{N-2} \nabla u_{n} \nabla u_{1} d x-\lambda \int_{\Omega}\left|u_{n}\right|^{q-2} u_{n} u_{1} d x-\int_{\Omega} g\left(u_{n}\right) u_{1} d x .
\end{aligned}
$$

Aplicando o Lema 2.6, deduzimos que

$$
\int_{\Omega}\left|\nabla u_{n}\right|^{N} d x=\int_{\Omega}\left|\nabla u_{1}\right|^{N} d x+o_{n}(1) .
$$

Portanto, $u_{n} \rightarrow u_{1}$ em $W_{0}^{1, N}(\Omega)$ com $u_{1}$ verificando $I_{\lambda}^{\prime}\left(u_{1}\right)=0$ e $I_{\lambda}\left(u_{1}\right)=c>0$. 


\subsubsection{Segunda solução}

Nesta seção, aplicamos o Princípio Variacional de Ekeland para provar a existência de uma uma solução fraca diferente da solução $u_{1}$ acima.

Lema 2.7 Existe $\lambda_{3}>0$ tal que, para $0<\lambda<\lambda_{3}$, o funcional $I_{\lambda}$ satisfaz a condição $(P . S)_{d}$ quando $d \leq 0$.

Demonstração. Seja $d \leq 0$ e suponha que $\left(u_{n}\right) \subset W_{0}^{1 . N}(\Omega)$ satisfaz

$$
I_{\lambda}\left(u_{n}\right) \rightarrow d \quad \text { e } I_{\lambda}^{\prime}\left(u_{n}\right) \rightarrow 0
$$

Queremos mostrar que $\left(u_{n}\right)$ admite uma subsequência fortemente convergente em $W_{0}^{1, N}(\Omega)$, para $\lambda<\lambda_{3}$.

Pela estimativa (2.8), $\left(u_{n}\right)$ verifica

$$
\left(\frac{1}{N}-\frac{1}{\nu}\right)\left\|u_{n}\right\|^{N} \leq \lambda\left(\frac{1}{q}-\frac{1}{\nu}\right) C\left\|u_{n}\right\|^{q}+d+o_{n}(1)\left\|u_{n}\right\|+o_{n}(1) .
$$

Logo, passando para uma subsequência se necessário, obtemos

$$
\left(\left(\frac{1}{N}-\frac{1}{\nu}\right) \limsup _{n \rightarrow \infty}\left\|u_{n}\right\|^{N-q}-\lambda\left(\frac{1}{q}-\frac{1}{\nu}\right) C\right) \limsup _{n \rightarrow \infty}\left\|u_{n}\right\|^{q} \leq 0,
$$

ou ainda,

$$
\limsup _{n \rightarrow \infty}\left\|u_{n}\right\|^{N} \leq\left(\lambda \frac{\left(\frac{1}{q}-\frac{1}{\nu}\right) C}{\left(\frac{1}{N}-\frac{1}{\nu}\right)}\right)^{\frac{N}{N-q}} .
$$

Escolhendo $\lambda_{3}:=\left(\frac{q(\nu-N)}{N C(\nu-q)}\right)\left(\left(\frac{\alpha_{N}}{\alpha_{0}}\right)^{N-1}\right)^{\frac{N-q}{N}}$, temos que para $\lambda<\lambda_{3}$,

$$
\limsup _{n \rightarrow \infty}\left\|u_{n}\right\|^{N}<\left(\frac{\alpha_{N}}{\alpha_{0}}\right)^{N-1} .
$$

Aplicando o Lema 2.5, segue que existem $\alpha>\alpha_{0}, t>1$ e $C>0$, independente de $n$, tais que

$$
\int_{\Omega} e^{t \alpha\left|u_{n}\right|^{N-1}} d x \leq C, \quad \forall n \geq n_{0}
$$

para algum $n_{0}$ suficientemente grande.

Uma vez que $\left(u_{n}\right)$ é limitada em $W_{0}^{1, N}(\Omega)$, existe $u \in W_{0}^{1, N}(\Omega)$ tal que

$$
u_{n} \rightarrow u \mathrm{em} W_{0}^{1, N}(\Omega)
$$




$$
u_{n}(x) \rightarrow u(x) \text { q.t.p. em } \Omega
$$

$\mathrm{e}$

$$
u_{n} \rightarrow u \text { em } L^{t}(\Omega), \text { para } t \geq 1
$$

Neste caso, o Lema 2.6 se aplica a sequência $\left(u_{n}\right)$, isto é:

(i) $\int_{\Omega}\left(g\left(u_{n}\right) u_{n}-g\left(u_{n}\right) u\right) d x=o_{n}(1)$

(ii) $\int_{\Omega}\left(\left|u_{n}\right|^{q}-\left|u_{n}\right|^{q-2} u_{n} u\right) d x=o_{n}(1)$;

(iii) $\nabla u_{n}(x) \rightarrow \nabla u(x)$ q.t.p. em $\Omega$;

(iv) $\int_{\Omega}\left|\nabla u_{n}\right|^{N-2} \nabla u_{n} \nabla v d x \rightarrow \int_{\Omega}|\nabla u|^{N-2} \nabla u \nabla v d x, \quad \forall v \in W_{0}^{1, N}(\Omega)$.

Argumentando de modo análogo à prova da Proposição 2.1 obtemos

$$
\int_{\Omega}\left|\nabla u_{n}\right|^{N} d x=\int_{\Omega}|\nabla u|^{N} d x+o_{n}(1)
$$

Portanto, $u_{n} \rightarrow u$ em $W_{0}^{1, N}(\Omega)$

Proposição 2.2 Suponha que g satisfaz $\left(H_{0}\right),\left(H_{c}\right),\left(H_{4}\right)$ e $\left(H_{5}\right)$. Então, existe uma constante $\Lambda_{2}>0$ tal que, para $0<\lambda<\Lambda_{2}$, o problema $\left(P_{2}\right)$ possui uma solução $u_{2}$ verificando $I_{\lambda}\left(u_{2}\right)<0$.

Demonstração. Recorde que, para $s \geq N$, existem constantes $C_{1}, C_{3}>0$ tais que

$$
I_{\lambda}(u) \geq \frac{1}{2 N}\|u\|^{N}-\frac{C_{1}}{q} \lambda\|u\|^{q}-C_{3}\|u\|^{s}, \quad \forall u \in W_{0}^{1, N}(\Omega),\|u\| \leq R,
$$

onde $R>0$ verifica $\alpha R^{\frac{N}{N-1}}<\alpha_{N}$ (ver 2.2 ). Observe que podemos fixar $0<\rho<R$ de modo que, se $\lambda<\lambda_{1}:=\frac{q}{4 N C_{2}} \rho^{N-q}$, então

$$
I_{\lambda}(u)>0, \quad\|u\|=\rho .
$$

Considerando o espaço métrico completo $\bar{B}_{\rho}(0):=\left\{u \in W_{0}^{1, N}(\Omega) ;\|u\| \leq \rho\right\}$ com a métrica dada por $d(u, v)=\|u-v\|$, podemos aplicar o Princípio Variacional de Ekeland (ver Apêndice, Teorema A.7 para obter uma sequência $\left(u_{n}\right) \subset \bar{B}_{\rho}(0)$ satisfazendo

$$
I_{\lambda}\left(u_{n}\right) \longrightarrow d:=\inf \left\{I_{\lambda}(u) ; u \in \bar{B}_{\rho}(0)\right\}
$$


e para cada $v \neq u_{n}$

$$
I_{\lambda}(v)-I_{\lambda}\left(u_{n}\right)+\frac{1}{n}\left\|u_{n}-v\right\|>0 .
$$

Observe que $d<0$ pois, para toda $v \in W_{0}^{1, N}(\Omega) \backslash\{0\}$ temos

$$
I_{\lambda}(t v) \leq \frac{t^{N}}{N}\|v\|^{N}-\frac{\lambda t^{q}}{q} \int_{\Omega}|v|^{q} d x<0
$$

para $t>0$ próximo de 0 .

Uma vez que $I_{\lambda}\left(u_{n}\right)>0$, para $\left\|u_{n}\right\|=\rho$, é fácil ver que, a menos de subsequência, $\left(u_{n}\right) \subset B_{\rho}(0)$. Seja $\varphi \in W_{0}^{1, N}(\Omega)$ e considere a sequência $v_{n}:=u_{n}+t \varphi \subset B_{\rho}(0), t \approx 0$. Por (2.16),

$$
\frac{1}{t}\left[I_{\lambda}\left(u_{n}+t \varphi\right)-I_{\lambda}\left(u_{n}\right)\right]>-\frac{1}{n}\|\varphi\| .
$$

$\operatorname{Logo}, I_{\lambda}^{\prime}\left(u_{n}\right) \varphi \geq-\frac{1}{n}\|\varphi\|$ e da mesma forma, $I_{\lambda}^{\prime}\left(u_{n}\right)(-\varphi) \geq-\frac{1}{n}\|\varphi\|$. Portanto,

$$
\left|I_{\lambda}^{\prime}\left(u_{n}\right) \varphi\right|<\frac{1}{n}\|\varphi\|, \quad \text { para toda } \varphi \in W_{0}^{1, N}(\Omega) .
$$

Consequentemente,

$$
\left\|I_{\lambda}^{\prime}\left(u_{n}\right)\right\| \longrightarrow 0, \text { quando } n \rightarrow+\infty \text {. }
$$

De 2.15) e 2.17), segue que $\left(u_{n}\right)$ é uma sequência $(P . S)_{d}$ para o funcional $I_{\lambda}$.

Fixe $\Lambda_{2}:=\min \left\{\lambda_{1}, \lambda_{3}\right\}$, onde $\lambda_{1}$ e $\lambda_{3}$ são obtidos nos Lemas 2.2 e 2.7, respectivamente. Aplicando o Lema 2.7, obtemos uma subsequência, ainda denotada por $\left(u_{n}\right)$, que converge fortemente para uma função $u_{2} \in W_{0}^{1, N}(\Omega)$. Neste caso,

$$
I_{\lambda}^{\prime}\left(u_{2}\right)=0 \text { e } I_{\lambda}\left(u_{2}\right)=d<0
$$

como queríamos provar.

Observação 2.1 Se u é uma solução não trivial de $\left(P_{2}\right)$, temos

$$
\left\|u^{-}\right\|=I_{\lambda}^{\prime}(u) u^{-}=0
$$

ou seja $u \geq 0$. Além disso, como consequência da Desigualdade de Harnack [78], segue que $u>0, e m \Omega$.

\section{Demonstração do Teorema 2}

Demonstração. Segue diretamente das Proposições 2.1 e 2.2 e da Observação 2.1 . 


\subsubsection{Multiplicidade de soluções com energia negativa}

Nesta seção, provamos o Teorema 4, que garante a existência de uma quantidade infinita de soluções para o problema $\left(P_{2}\right)$. Para isto, admitimos que $g$ é uma função ímpar, assim, não será assumido que $g(s)=0$, para $s<0$. O funcional associado ao problema $\left(P_{2}\right)$, ainda denotado por $I_{\lambda}$, é definido por

$$
I_{\lambda}(u)=\frac{1}{N} \int_{\Omega}|\nabla u|^{N} d x-\frac{\lambda}{q} \int_{\Omega}|u|^{q} d x-\int_{\Omega} G(u) d x .
$$

Usamos argumentos que podem ser encontrados em [17] e consiste em obter pontos críticos para uma forma truncada do funcional energia associado. Iniciamos este estudo observando que vale a seguinte estimativa (ver (2.2),

$$
I_{\lambda}(u) \geq \frac{1}{2 N}\|u\|^{N}-\frac{C_{1}}{q} \lambda\|u\|^{q}-C_{3}\|u\|^{s}, \quad \forall u \in W_{0}^{1, N}(\Omega),\|u\| \leq R,
$$

onde $R>0$ verifica $\alpha R^{\frac{N}{N-1}}<\alpha_{N}$ e $s>N$. Fixamos $0<R_{1}<R$ suficientemente pequeno tal que

$$
\frac{1}{4 N} R_{1}^{N}-C_{3} R_{1}^{s}>0
$$

Considere $\lambda<\lambda_{1}:=\frac{q}{4 N C_{1}} R_{1}^{N-q}$ e defina

$$
l(x)=\frac{1}{2 N} x^{N}-\frac{C_{1}}{q} \lambda_{1} x^{q}-C_{3} x^{s}, \quad \forall x \geq 0 .
$$

Observe que

$$
I_{\lambda}(u) \geq l(\|u\|), \text { para }\|u\|<R_{1} \text { e } \forall \lambda<\lambda_{1} .
$$

Sendo $q<N$, temos que $l(x)<0$, para $x \approx 0$ e pela definição de $\lambda_{1}$, segue que $l\left(R_{1}\right)>0$. Assim, considerando $R_{0}:=\max \left\{0<x \leq R_{1} ; l(x) \leq 0\right\}$, temos que $l\left(R_{0}\right)=0$.

Note que podemos escolher uma função $\tau:[0,+\infty) \rightarrow[0,1]$ de classe $C^{\infty}$ verificando

$$
\begin{aligned}
& \tau(x)=1, \text { se } x \leq R_{0} \\
& \tau(x)=0, \text { se } x \geq R_{1} .
\end{aligned}
$$

Defina

$$
\tilde{l}(x)=\frac{1}{2 N} x^{N}-\frac{C_{1}}{q} \lambda_{1} x^{q}-C_{3} x^{s} \tau(x)
$$

e

$$
\tilde{I}_{\lambda}(u)=\frac{1}{N}\|u\|^{N}-\frac{\lambda}{q}|u|_{q}^{q}-\tau(u) \int_{\Omega} G(u) d x
$$

onde $\tau(u)=\tau(\|u\|)$. 
Note que pela escolha de $\lambda$ temos que para $\|u\| \geq R_{1}$,

$$
\tilde{l}(\|u\|)=\frac{1}{2 N}\|u\|^{N}-\frac{C_{1} \lambda_{1}}{q}\|u\|^{q}>0
$$

e pela definição de $R_{0}$,

$$
\tilde{l}(\|u\|)<0 \Rightarrow\|u\|<R_{0}
$$

Além disso,

$$
\tilde{I}_{\lambda}(u) \geq \tilde{l}(\|u\|), \quad \forall u \in W_{0}^{1, N}(\Omega) .
$$

O próximo lema garante que valores críticos negativos para o funcional truncado $\tilde{I}_{\lambda}$, são também valores críticos negativos para $I_{\lambda}$.

Lema 2.8 As seguintes afirmações são verdadeiras:

(i) $\tilde{I}_{\lambda} \in C^{1}\left(W_{0}^{1, N}(\Omega), \mathbb{R}\right)$;

(ii) se $\tilde{I}_{\lambda}(u)<0$, então $\|u\|<R_{0}$

(iii) $I_{\lambda}(v)=\tilde{I}_{\lambda}(v)$, para cada $v$ suficientemente próximo de u com $\|u\|<R_{0}$;

(iv) $\tilde{I}_{\lambda}$ satisfaz a condição $(P . S)_{d}$ com $d<0$, para $\lambda<\lambda_{3}$.

Demonstração. Observe que $(i)$ e $(i i i)$ são imediatos da definição de $\tilde{I}_{\lambda}$. Se $\tilde{I}_{\lambda}(u)<0$ então, $\tilde{l}(\|u\|)<0$. Pela escolha de $R_{0}$ temos que $\|u\|<R_{0}$, o que prova $(i i)$.

Para justificar $(i v)$, considere $\left(u_{n}\right) \subset W_{0}^{1, N}(\Omega)$ uma sequência $(P . S)_{d}$ para $\tilde{I}_{\lambda}$. Sem perda de generalidade, $\tilde{I}_{\lambda}\left(u_{n}\right) \leq \frac{d}{2}<0$, de onde segue que $\left\|u_{n}\right\|<R_{0}$. Portanto, pelo item (iii)

$$
\tilde{I}_{\lambda}\left(u_{n}\right)=I_{\lambda}\left(u_{n}\right) \quad \text { e } \quad \tilde{I}_{\lambda}^{\prime}\left(u_{n}\right)=I_{\lambda}^{\prime}\left(u_{n}\right) .
$$

Aplicando o Lema 2.7, segue que $\tilde{I}_{\lambda}$ verifica a condição $(P . S)_{d}$.

Lema 2.9 Para cada $n \in \mathbb{N}$, existe $\epsilon>0$ tal que

$$
\gamma\left(\tilde{I}_{\lambda}^{-\epsilon}\right) \geq n
$$

onde

$$
\tilde{I}_{\lambda}^{-\epsilon}=\left\{u \in W_{0}^{1, N}(\Omega) ; \tilde{I}_{\lambda}(u) \leq-\epsilon\right\}
$$

e $\gamma$ denota o gênero do conjunto $\tilde{I}_{\lambda}^{-\epsilon}$ (ver Apêndice). 
Demonstração. Fixe $n \in \mathbb{N}$ e seja $E_{n}$ um subespaço $n$-dimensional de $W_{0}^{1, N}(\Omega)$. Para $u_{n} \in E_{n} \operatorname{com}\left\|u_{n}\right\|=1$ e $0<\rho<R_{0}$, temos

$$
\tilde{I}_{\lambda}\left(\rho u_{n}\right)=I_{\lambda}\left(\rho u_{n}\right)=\frac{1}{N} \rho^{N}-\frac{\lambda}{q} \rho^{q}\left|u_{n}\right|_{q}^{q}-\int_{\Omega} G\left(\rho u_{n}\right) d x \leq \frac{1}{N} \rho^{N}-\frac{\lambda}{q} \rho^{q}\left|u_{n}\right|_{q}^{q} .
$$

Considerando

$$
\beta_{n}=\inf \left\{|u|_{q}^{q} ; u \in E_{n},\|u\|=1\right\}>0
$$

temos que

$$
\tilde{I}_{\lambda}\left(\rho u_{n}\right) \leq \frac{1}{N} \rho^{N}-\frac{\lambda}{q} \rho^{q} \beta_{n}
$$

Note que podemos escolher $\delta<R_{0}$ e $\epsilon=\epsilon(n)>0$ tal que

$$
\tilde{I}_{\lambda}\left(\delta u_{n}\right) \leq-\epsilon
$$

Assim, para cada $n$, temos

$$
\left(\partial B_{\delta}(0) \cap E_{n}\right) \subset \tilde{I}_{\lambda}^{-\epsilon}
$$

e segue das propriedades de gênero que

$$
\gamma\left(\tilde{I}_{\lambda}^{-\epsilon}\right) \geq \gamma\left(\partial B_{\delta}(0) \cap E_{n}\right)=n .
$$

A demonstração do Teorema 4 segue com as mesmas idéias encontradas em [17].

\section{Demonstração do Teorema 4}

Iniciamos fazendo algumas definições

$$
\begin{gathered}
\Sigma_{k}:=\left\{A \subset W_{0}^{1, N}(\Omega) \backslash\{0\} ; A \text { é fechado }, A=-A \text { e } \gamma(A) \geq k\right\}, \\
c_{k}:=\inf _{A \in \Sigma_{k}} \sup _{u \in A} \tilde{I}_{\lambda}(u), \\
K_{c}:=\left\{u \in W_{0}^{1, N}(\Omega) ; \tilde{I}_{\lambda}^{\prime}(u)=0 \text { e } \tilde{I}_{\lambda}(u)=c\right\} .
\end{gathered}
$$

Queremos mostrar que se $c=c_{k}=c_{k+1}=\ldots=c_{k+r}$, então

$$
\gamma\left(K_{c}\right) \geq r+1
$$

Em particular, $c_{k}$ é valor crítico.

Primeiramente, vejamos que $-\infty<c_{k}<0$. De fato, pelo Lema 2.9 temos que, para cada $k \in \mathbb{N}$, existe $\epsilon>0$ tal que $\gamma\left(\tilde{I}_{\lambda}^{-\epsilon}\right) \geq k$. Uma vez que $\tilde{I}_{\lambda}$ é contínuo e par, segue que $\tilde{I}_{\lambda}^{-\epsilon} \in \Sigma_{k}$. Portanto,

$$
c_{k} \leq \sup _{u \in \tilde{I}_{\lambda}^{-\epsilon}} \tilde{I}_{\lambda}(u) \leq-\epsilon<0, \quad \forall k \in \mathbb{N}
$$


Sendo $\tilde{I}_{\lambda}$ limitado inferiormente segue que $c_{k}>-\infty$.

Uma vez que $c<0, \tilde{I}_{\lambda}$ verifica a condição $(P . S)_{c}$, ou seja, $K_{c}$ é compacto e claramente é simétrico, logo, $\gamma\left(K_{c}\right)$ está bem definido.

Vamos supor por contradição que

$$
c=c_{k}=c_{k+1}=\ldots=c_{k+r} \quad \text { e } \quad \gamma\left(K_{c}\right)<r+1 .
$$

Pelas propriedades de gênero (ver Apêndice) existe uma vizinhança $\mathcal{K}$ de $K_{c} \operatorname{com} \gamma(\mathcal{K})=$ $\gamma\left(K_{c}\right)<r+1$. Usando o Lema de deformação (ver Apêndice), segue que existe um homeomorfismo ímpar $\eta: W_{0}^{1, N}(\Omega) \rightarrow W_{0}^{1, N}(\Omega)$ satisfazendo

$$
\eta\left(\tilde{I}_{\lambda}^{c+\delta} \backslash \mathcal{K}\right) \subset \tilde{I}_{\lambda}^{c-\delta}
$$

onde $0<\delta<-c$ pois, $\tilde{I}_{\lambda}$ verifica a condição $(P . S)$ em $\tilde{I}_{\lambda}^{0}$. Por hipótese,

$$
c=c_{k+r}=\inf _{A \in \Sigma_{k+r}} \sup _{u \in A} \tilde{I}_{\lambda}(u) .
$$

Portanto, existe $A \in \Sigma_{k+r}$ tal que

$$
\sup _{u \in A} \tilde{I}_{\lambda}(u) \leq c+\delta
$$

consequentemente, $A \subset \tilde{I}_{\lambda}^{c+\delta}$. Logo, segue de 2.18 que

$$
\eta(A \backslash \mathcal{K}) \subset \eta\left(\tilde{I}_{\lambda}^{c+\delta} \backslash \mathcal{K}\right) \subset \tilde{I}_{\lambda}^{c-\delta}
$$

Mas, pelas propriedades de gênero

$$
\gamma(\overline{\eta(A \backslash \mathcal{K})}) \geq \gamma(\overline{A \backslash \mathcal{K}}) \geq \gamma(A)-\gamma(\mathcal{K}) \geq(k+r)-r=k .
$$

De onde segue que

$$
\overline{\eta(A \backslash \mathcal{K})} \in \Sigma_{k}
$$

Logo,

$$
\sup _{u \in \overline{\eta(A \backslash \mathcal{K})}} \tilde{I}_{\lambda}(u) \geq c_{k}=c,
$$

o que contradiz 2.19). Portanto, se $c=c_{k}=c_{k+1}=\ldots=c_{k+r}$, então $\gamma\left(K_{c}\right) \geq r+1$. Observe que isto assegura que $c_{k}$ é valor crítico pois $\gamma\left(K_{c_{k}}\right) \geq 1$, ou seja, $K_{c_{k}}$ é não vazio, para todo $k \in \mathbb{N}$. Além disso, se os valores $c_{k}$ não forem todos distintos, teremos $\gamma\left(K_{c}\right)>1$ e isto significa que $K_{c}$ é um conjunto infinito. Desta forma, chegamos a uma quantidade infinita de pontos críticos de $\tilde{I}_{\lambda}$ com energia negativa, para $\lambda<\hat{\Lambda}:=\min \left\{\lambda_{1}, \lambda_{3}\right\}$. Pelo Lema 2.7, estes pontos são pontos críticos de $I_{\lambda}$. Isto mostra a existência de uma quantidade infinita de soluções fracas para o problema $\left(P_{2}\right)$. 


\subsection{Soluções para uma classe de equações em $\mathbb{R}^{N}$}

Nesta seção, consideramos o problema

$$
\left\{\begin{array}{l}
-\Delta_{N} u+|u|^{N-2} u=\lambda h(x)|u|^{q-2} u+g(u), \quad x \in \mathbb{R}^{N}, \\
u \in W^{1, N}\left(\mathbb{R}^{N}\right),
\end{array}\right.
$$

onde $N \geq 2,1<q<N, \lambda>0$ e $h$ é uma função positiva que pertence a $L^{\theta}\left(\mathbb{R}^{N}\right)$ com $\theta=\frac{N}{N-q}$. Como dito na Introdução, para este problema vamos supor as hipóteses $\left(H_{c}\right)$, $\left(H_{0}\right),\left(H_{4}\right)$ e acrescentamos as seguintes hipóteses:

$\left(\mathbf{H}_{\mathbf{6}}\right) g \in C^{1}(\mathbb{R}, \mathbb{R})$ é tal que $\frac{g(s)}{s^{N-1}}$ é crescente em $(0,+\infty) ;$

$\left(\mathbf{H}_{\mathbf{7}}\right)$ existe $C>0$ tal que

$$
\left|g^{\prime}(s)\right| \leq C e^{\alpha_{0}|s|^{N-1}}, \quad \forall s \in \mathbb{R}
$$

A condição $\left(H_{5}\right)$ será considerada com $\Omega=\mathbb{R}^{N}$, ou seja,

$\left(\mathbf{H}_{\mathbf{5}}\right)$ Existem $p>N$ e uma constante $C_{p}>0$ tais que

$$
g(s) \geq C_{p} s^{p-1}, \quad \forall s \geq 0,
$$

onde

com

$$
C_{p}>S_{p}^{p}\left\{\frac{p(\nu-N)}{8^{N} \nu(p-N)}\left(\frac{\alpha_{N}}{\alpha_{0}}\right)^{N-1}\right\}^{\frac{N-p}{N}}
$$

$$
S_{p}:=\inf _{v \in W^{1, N}\left(\mathbb{R}^{N}\right) \backslash\{0\}} \frac{\|v\|_{W^{1, N}\left(\mathbb{R}^{N}\right)}}{|v|_{L^{p}\left(\mathbb{R}^{N}\right)}} .
$$

No espaço de Sobolev $W^{1, N}\left(\mathbb{R}^{N}\right)$ vamos considerar a norma usual, isto é,

$$
\|u\|=\left(\int_{\mathbb{R}^{N}}\left(|\nabla u|^{N}+|u|^{N}\right) d x\right)^{1 / N}, \quad u \in W^{1, N}\left(\mathbb{R}^{N}\right) .
$$

Assumimos que $g(s)=0$, para $s<0$, pois queremos encontrar soluções positivas. Provaremos a existência de uma solução com energia negativa e outra com energia positiva, quando $\lambda$ está abaixo de um determinado $\Lambda>0$. Uma vez que faremos uma abordagem variacional, encontrar tais soluções se resume a provar a existência de pontos críticos para o funcional $I_{\lambda}: W^{1, N}\left(\mathbb{R}^{N}\right) \rightarrow \mathbb{R}$ definido por

$$
I_{\lambda}(u)=\frac{1}{N}\|u\|^{N}-\frac{\lambda}{q} \int_{\mathbb{R}^{N}} h(x) u_{+}^{q} d x-\int_{\mathbb{R}^{N}} G(u) d x .
$$


O funcional $I_{\lambda}$ está bem definido, pertence a $C^{1}\left(W^{1, N}\left(\mathbb{R}^{N}\right), \mathbb{R}\right)$ e sua derivada é dada por

$$
I_{\lambda}^{\prime}(u) v=\int_{\mathbb{R}^{N}}\left(|\nabla u|^{N-2} \nabla u \nabla v-|u|^{N-2} u v\right) d x-\lambda \int_{\mathbb{R}^{N}} h(x) u_{+}^{q-1} v d x-\int_{\mathbb{R}^{N}} g(u) v d x,
$$

para toda $u, v \in W^{1, N}\left(\mathbb{R}^{N}\right)$.

Lema 2.10 Existe $\lambda_{1}>0$ tal que, para cada $\lambda<\lambda_{1}$, o funcional $I_{\lambda}$ satisfaz as seguintes condições:

(i) Existem $\beta, \rho>0$ tais que

$$
I_{\lambda}(u) \geq \beta, \text { para }\|u\|=\rho .
$$

(ii) Existe e $\in W^{1, N}\left(\mathbb{R}^{N}\right)$ tal que $\|e\|>\rho$ e $I_{\lambda}(e)<0$.

Demonstração. Observe inicialmente que pelas hipóteses $\left(H_{0}\right)$ e $\left(H_{c}\right)$, temos que dados $\epsilon>0$ e $s \geq 1$, existe $C_{\epsilon}=C(\epsilon, s)>0$ tal que, para cada $\alpha>\alpha_{0}$,

$$
|G(t)| \leq \frac{\epsilon}{N}|t|^{N}+C_{\epsilon}|t|^{s}\left(e^{\alpha|t|^{N-1}}-S_{N-2}(\alpha, t)\right), \quad \forall t \in \mathbb{R} .
$$

Consequentemente,

$$
I_{\lambda}(u) \geq \frac{1}{N}\|u\|^{N} d x-\frac{\lambda}{q} \int_{\mathbb{R}^{N}} h(x) u_{+}^{q} d x-\frac{\epsilon}{N}|u|_{N}^{N}-C_{\epsilon} \int_{\mathbb{R}^{N}}|u|^{s}\left(e^{\alpha|u|^{\frac{N}{N-1}}}-S_{N-2}(\alpha, u)\right) d x .
$$

A Desigualdade de Hölder e a imersão de Sobolev $W^{1, N}\left(\mathbb{R}^{N}\right) \hookrightarrow L^{t}\left(\mathbb{R}^{N}\right), N \leq t<\infty$ na estimativa anterior resulta em

$$
I_{\lambda}(u) \geq \frac{1}{N}\|u\|^{N} d x-\frac{C_{1}}{q} \lambda|h|_{\theta}\|u\|^{q}-\frac{\epsilon C_{2}}{N}\|u\|^{N}-C_{\epsilon}|u|_{s t_{1}}^{s}\left|e^{\alpha|u|^{\frac{N}{N-1}}}-S_{N-2}(\alpha, u)\right|_{t_{2}} .
$$

Com o mesmo raciocínio aplicado em (1.4) conclui-se que

$$
I_{\lambda}(u) \geq \frac{1}{N}\|u\|^{N} d x-\frac{C_{1}}{q} \lambda|h|_{\theta}\|u\|^{q}-\frac{\epsilon C_{2}}{N}\|u\|^{N}-C_{3}\|u\|^{s},
$$

para toda $u \in W^{1, N}\left(\mathbb{R}^{N}\right)$ com $\|u\| \leq R$, onde $R>0$ verifica $\alpha R^{\frac{N}{N-1}}<\alpha_{N}$. Escolhendo $\epsilon=\frac{1}{2 C_{2}}$, podemos escrever

$$
I_{\lambda}(u) \geq \frac{1}{2 N}\|u\|^{N}-\frac{C_{1}}{q} \lambda|h|_{\theta}\|u\|^{q}-C_{3}\|u\|^{s}, \quad \forall u \in W^{1, N}\left(\mathbb{R}^{N}\right), \quad\|u\| \leq R .
$$


Considerando $s>N$, podemos fixar $0<\rho<R$ suficientemente pequeno verificando

$$
\beta:=\frac{1}{4 N} \rho^{N}-C_{3} \rho^{s}>0
$$

Observe que se $\lambda<\lambda_{1}:=\frac{q}{4 N C_{1}|h|_{\theta}} \rho^{N-q}$, então para $\|u\|=\rho$ temos

$$
I_{\lambda}(u)>\frac{1}{2 N} \rho^{N}-\frac{C_{2}|h|_{\theta}}{q} \frac{q}{4 N C_{2}|h|_{\theta}} \rho^{N-q} \rho^{q}-C_{3} \rho^{s}=\frac{1}{4 N} \rho^{N}-C_{3} \rho^{s} .
$$

De onde segue que,

$$
I_{\lambda}(u)>\beta>0, \quad\|u\|=\rho,
$$

provando $(i)$. A justificativa de (ii) é semelhante a prova do Lema 2.2(ii) e será omitida.

\subsubsection{Primeira solução}

Nosso objetivo é provar a existência de uma solução com energia positiva para o problema $\left(P_{3}\right)$. Iniciamos com o seguinte lema.

Lema 2.11 Se u é um ponto crítico de $I_{\lambda}$, então existe $K>0$ dependendo apenas de $q, \nu$ e $h$ tal que

$$
-I_{\lambda}(u) \leq K \lambda^{\theta}
$$

Demonstração. Seja $u$ um ponto crítico de $I_{\lambda}$. Observe que, por $\left(H_{4}\right)$ e a Desigualdade de Young,

$$
\begin{aligned}
I_{\lambda}(u) & =I_{\lambda}(u)-\frac{1}{\nu} I_{\lambda}^{\prime}(u) u \geq\left(\frac{1}{N}-\frac{1}{\nu}\right)\|u\|^{N}-\left(\frac{1}{q}-\frac{1}{\nu}\right) \int_{\mathbb{R}^{N}} \lambda h(x) u_{+}^{q} d x \\
& \geq\left(\frac{1}{N}-\frac{1}{\nu}\right)\|u\|^{N}-\left(\frac{1}{q}-\frac{1}{\nu}\right) \int_{\mathbb{R}^{N}}\left(\epsilon u_{+}^{N}+C_{\epsilon} \lambda^{\theta} h(x)^{\theta}\right) d x \\
& \geq\left[\left(\frac{1}{N}-\frac{1}{\nu}\right)-\epsilon C\left(\frac{1}{q}-\frac{1}{\nu}\right)\right]\|u\|^{N}-C_{\epsilon}\left(\frac{1}{q}-\frac{1}{\nu}\right)|h|_{\theta}^{\theta} \lambda^{\theta} .
\end{aligned}
$$

Escolhendo $\epsilon$ suficientemente pequeno, segue que

$$
I_{\lambda}(u) \geq C\|u\|^{N}-\mathrm{K} \lambda^{\theta} \geq-\mathrm{K} \lambda^{\theta},
$$

onde $\mathrm{K}:=C_{\epsilon}\left(\frac{1}{q}-\frac{1}{\nu}\right)|h|_{\theta}^{\theta}$. 
Considere o funcional $I_{\infty}: W^{1, N}\left(\mathbb{R}^{N}\right) \rightarrow \mathbb{R}$ dado por

$$
I_{\infty}(u)=\frac{1}{N}\|u\|^{N}-\int_{\mathbb{R}^{N}} G(u) d x .
$$

As condições de crescimento sobre $g$, asseguram que $I_{\infty}$ satisfaz a geometria do Teorema do Passo da Montanha. Vamos denotar por $c_{\infty}$ o nível do Passo da Montanha para o funcional $I_{\infty}$. Um fato importante é que $I_{\infty}$ possui um ponto crítico não trival com energia mínima, mais precisamente, existe $0<\Psi \in W^{1, N}\left(\mathbb{R}^{N}\right) \backslash\{0\}$ que verifica

$$
I_{\infty}^{\prime}(\Psi)=0 \quad \text { e } \quad I_{\infty}(\Psi)=c_{\infty}=\inf _{u \in \mathcal{M}_{\infty}} I_{\infty}(u)
$$

onde $\mathcal{M}_{\infty}=\left\{u \in W^{1, N}\left(\mathbb{R}^{N}\right) \backslash\{0\} ; I_{\infty}^{\prime}(u) u=0\right\}$. Estas informações podem ser encontradas em Alves \& Figueiredo [12].

Considerando a hipótese $\left(H_{5}\right)$, podemos justificar com raciocínio semelhante ao aplicado na prova do Lema 2.3, que vale a seguinte estimativa

$$
c_{\infty}<\frac{1}{8^{N}}\left(\frac{1}{N}-\frac{1}{\nu}\right)\left(\frac{\alpha_{N}}{\alpha_{0}}\right)^{N-1}
$$

Proposição 2.3 Seja $c<c_{\infty}-K \lambda^{\theta}$, com $K$ dado no Lema 2.11. Então, I $I_{\lambda}$ satisfaz a condição $(P . S)_{c}$.

Demonstração. Seja $\left(u_{n}\right) \subset W^{1, N}\left(\mathbb{R}^{N}\right)$ uma sequência $(P . S)_{c}$ para o funcional $I_{\lambda}$, isto é,

$$
I_{\lambda}\left(u_{n}\right) \rightarrow c \quad \text { e } \quad I_{\lambda}^{\prime}\left(u_{n}\right) \rightarrow 0, \quad \text { quando } n \rightarrow+\infty
$$

Repetindo os cálculos feitos em (2.21) para a sequência $\left(u_{n}\right)$ e usando 2.23 encontramos

$$
\left[\left(\frac{1}{N}-\frac{1}{\nu}\right)-\epsilon C\left(\frac{1}{q}-\frac{1}{\nu}\right)\right]\left\|u_{n}\right\|^{N}-C_{\epsilon}\left(\frac{1}{q}-\frac{1}{\nu}\right) \lambda^{\theta}|h|_{\theta}^{\theta} \leq c+o_{n}(1)\left\|u_{n}\right\| .
$$

Escolhendo $\epsilon=\frac{\left(\frac{1}{N}-\frac{1}{\nu}\right)}{C\left(\frac{1}{q}-\frac{1}{\nu}\right)}\left(1-\frac{1}{2^{N}}\right)>0$, obtemos

$$
\frac{1}{2^{N}}\left(\frac{1}{N}-\frac{1}{\nu}\right)\left\|u_{n}\right\|^{N} \leq c+\mathrm{K} \lambda^{\theta}+o_{n}(1)\left\|u_{n}\right\|<c_{\infty}+o_{n}(1)\left\|u_{n}\right\| .
$$

Portanto,

$$
\limsup _{n \rightarrow \infty}\left\|u_{n}\right\|^{N} \leq \frac{c_{\infty}}{\frac{1}{2^{N}}\left(\frac{1}{N}-\frac{1}{\nu}\right)}
$$

e de 2.22 segue que

$$
\limsup _{n \rightarrow \infty}\left\|u_{n}\right\|^{N}<\frac{1}{4^{N}}\left(\frac{\alpha_{N}}{\alpha_{0}}\right)^{N-1}
$$


Pela limitação da sequência $\left(u_{n}\right)$, extraindo uma subsequência se necessário, segue a existência de uma função $u \in W^{1, N}\left(\mathbb{R}^{N}\right)$ verificando

$$
\left\{\begin{array}{cl}
u_{n} \rightarrow u & \text { em } W^{1, N}\left(\mathbb{R}^{N}\right), \\
u_{n}(x) \rightarrow u(x) & \text { q. t. p. em } \mathbb{R}^{N}, \\
u_{n} \rightarrow u & \text { em } L_{l o c}^{t}\left(\mathbb{R}^{N}\right), \text { para } t \geq N .
\end{array}\right.
$$

Vamos supor, sem perda de generalidade, que $u_{n} \geq 0, \forall n \in \mathbb{N}$, pois é possível verificar que a sequência $\left(\left(u_{n}\right)_{+}\right)$também é uma sequência $(P . S)_{c}$ para $I_{\lambda}$.

Os seguintes limites podem ser provados com os mesmos argumentos explorados no Lema 1.8.

(i) $\nabla u_{n}(x) \rightarrow \nabla u(x)$ q.t.p. em $\mathbb{R}^{N}$;

(ii) $\int_{\mathbb{R}^{N}}\left|\nabla u_{n}\right|^{N-2} \nabla u_{n} \nabla v d x \rightarrow \int_{\mathbb{R}^{N}}|\nabla u|^{N-2} \nabla u \nabla v d x, \quad \forall v \in W^{1, N}\left(\mathbb{R}^{N}\right) ;$

$($ iii $) \int_{\mathbb{R}^{N}}\left|u_{n}\right|^{N-2} u_{n} v d x \rightarrow \int_{\mathbb{R}^{N}}|u|^{N-2} u v d x, \quad \forall v \in W^{1, N}\left(\mathbb{R}^{N}\right) ;$

(iv) $\int_{\mathbb{R}^{N}} g\left(u_{n}\right) \varphi d x \rightarrow \int_{\mathbb{R}^{N}} g(u) \varphi d x, \forall \varphi \in C_{0}^{\infty}\left(\mathbb{R}^{N}\right) ;$

(v) $\int_{\mathbb{R}^{N}} h(x) u_{n}^{q-1} \varphi d x \rightarrow \int_{\mathbb{R}^{N}} h(x) u^{q-1} \varphi d x, \quad \forall \varphi \in C_{0}^{\infty}\left(\mathbb{R}^{N}\right)$.

Justificaremos apenas o item $(v)$. Observe que, para $\varphi \in C_{0}^{\infty}\left(\mathbb{R}^{N}\right)$, temos

$$
\begin{aligned}
\left|\int_{\mathbb{R}^{N}} h\left(u_{n}^{q-1}-u^{q-1}\right) \varphi d x\right| & =\left|\int_{\mathbb{R}^{N}} h^{\frac{q-1}{q}}\left(u_{n}^{q-1}-u^{q-1}\right) h^{\frac{1}{q}} \varphi d x\right| \\
& \leq\left(\int_{\mathbb{R}^{N}} h\left|u_{n}^{q-1}-u^{q-1}\right|^{\frac{q}{q-1}} d x\right)^{\frac{q-1}{q}}\left(\int_{\mathbb{R}^{N}} h \varphi^{q} d x\right)^{\frac{1}{q}} .
\end{aligned}
$$

Considerando $U_{n}:=\left|u_{n}^{q-1}-u^{q-1}\right|^{\frac{q}{q-1}}$, podemos escrever

$$
\left|\int_{\mathbb{R}^{N}} h\left(u_{n}^{q-1}-u^{q-1}\right) \varphi d x\right| \leq\left(\int_{\mathbb{R}^{N}} h U_{n} d x\right)^{\frac{q-1}{q}}\left(\int_{\mathbb{R}^{N}} h \varphi^{q} d x\right)^{\frac{1}{q}} .
$$

Note que $\left(U_{n}\right)$ é limitada em $L^{\frac{N}{q}}$ e $U_{n}(x) \rightarrow 0$ q.t.p. em $\mathbb{R}^{N}$. Uma vez que $h \in L^{\frac{N}{N-q}}$, um Lema de Brezis-Lieb (ver Apêndice) assegura que

$$
\int_{\mathbb{R}^{N}} h U_{n} d x=o_{n}(1)
$$

o que juntamente com 2.27) conclui a prova de $(v)$. 
Usando estas informações, mostra-se que $I_{\lambda}^{\prime}(u) \varphi=0$, para cada $\varphi \in C_{0}^{\infty}\left(\mathbb{R}^{N}\right)$. De onde concluímos que $I_{\lambda}^{\prime}(u)=0$.

Defina

$$
v_{n}:=u_{n}-u
$$

Neste caso,

$$
\begin{cases}v_{n} \rightarrow 0 \quad \text { em } W^{1, N}\left(\mathbb{R}^{N}\right), \\ v_{n} \rightarrow 0 \quad \text { em } L_{l o c}^{t}\left(\mathbb{R}^{N}\right), \text { para } t \geq N\end{cases}
$$

Aplicando o Lema de Brezis-Lieb (ver Apêndice), temos

$$
\left\|v_{n}\right\|^{N}=\left\|u_{n}\right\|^{N}-\|u\|^{N}+o_{n}(1)
$$

De 2.26$)$ e 2.28 obtemos

$$
\limsup _{n \rightarrow \infty}\left\|v_{n}\right\|^{N}<\frac{1}{4^{N}}\left(\frac{\alpha_{N}}{\alpha_{0}}\right)^{N-1}
$$

Vamos agora justificar cada uma das seguintes afirmações:

(a) $\int_{\mathbb{R}^{N}}\left|G\left(v_{n}\right)-G\left(u_{n}\right)+G(u)\right| d x=o_{n}(1) ;$

(b) existe $r>1$ tal que $\int_{\mathbb{R}^{N}}\left|g\left(v_{n}\right)-g\left(u_{n}\right)+g(u)\right|^{r} d x=o_{n}(1)$;

(c) $\int_{\mathbb{R}^{N}} h(x)\left(v_{n}^{+}\right)^{q} d x=\int_{\mathbb{R}^{N}} h(x) u_{n}^{q} d x-\int_{\mathbb{R}^{N}} h(x) u^{q} d x+o_{n}(1) ;$

(d) $I_{\lambda}\left(v_{n}\right)=I_{\lambda}\left(u_{n}\right)-I_{\lambda}(u)+o_{n}(1)$

(e) $\left\|I_{\lambda}^{\prime}\left(v_{n}\right)-I_{\lambda}^{\prime}\left(u_{n}\right)+I_{\lambda}^{\prime}(u)\right\|=o_{n}(1)$.

Análise de $(a)$. Observe que

$$
\left|G\left(v_{n}+u\right)-G\left(v_{n}\right)\right|=\left|\int_{0}^{1} \frac{d}{d t} G\left(v_{n}+t u\right) d t\right| \leq \int_{0}^{1}\left|g\left(v_{n}+t u\right) u\right| d t .
$$

Assim, dados $s \geq 1$ e $\alpha>\alpha_{0}$, existe uma constante $C=C(s)>0$ tal que

$\left|G\left(v_{n}+u\right)-G\left(v_{n}\right)\right| \leq\left|v_{n}+t u\right|^{N-1}|u|+C\left|v_{n}+t u\right|^{s}|u|\left[e^{\alpha\left(\left|v_{n}\right|+|u|\right)^{\frac{N}{N-1}}}-S_{N-2}\left(\alpha,\left(\left|v_{n}\right|+|u|\right)\right)\right]$.

Para simplificar, usaremos as notações $w_{n}:=\left|v_{n}\right|+|u|, Q_{n}:=\left[e^{\alpha\left|w_{n}\right|^{\frac{N}{N-1}}}-S_{N-2}\left(\alpha, w_{n}\right)\right] \mathrm{e}$ $Q:=\left[e^{\alpha|u|^{\frac{N}{N-1}}}-S_{N-2}(\alpha, u)\right]$. Desta forma,

$$
\left|G\left(v_{n}+u\right)-G\left(v_{n}\right)\right| \leq C\left|v_{n}\right|^{N-1}|u|+C|u|^{N}+C\left|v_{n}\right|^{s}|u| Q_{n}+C|u|^{s+1} Q_{n} .
$$


Portanto,

$\left|G\left(v_{n}+u\right)-G\left(v_{n}\right)-G(u)\right| \leq C\left|v_{n}\right|^{N-1}|u|+C|u|^{N}+C\left|v_{n}\right|^{s}|u| Q_{n}+C|u|^{s+1} Q_{n}+C|u|^{s} Q$.

Observe que por $(2.28)$ e 2.26$)$

$$
\|u\|^{N} \leq \limsup _{n \rightarrow \infty}\left[\|u\|^{N}+\left\|v_{n}\right\|^{N}\right]=\limsup _{n \rightarrow \infty}\left[\left\|u_{n}\right\|^{N}+o_{n}(1)\right]<\frac{1}{4^{N}}\left(\frac{\alpha_{N}}{\alpha_{0}}\right)^{N-1} .
$$

Logo, usando 2.29 temos

$$
\limsup _{n \rightarrow \infty}\left\|w_{n}\right\|<\left(\frac{1}{4}+\frac{1}{4}\right)\left(\frac{\alpha_{N}}{\alpha_{0}}\right)^{\frac{N-1}{N}},
$$

ou seja,

$$
\limsup _{n \rightarrow \infty}\left\|w_{n}\right\|^{N}<\left(\frac{\alpha_{N}}{\alpha_{0}}\right)^{N-1}
$$

Definindo

$$
P_{n}:=C\left|v_{n}\right|^{N-1}|u|+C|u|^{N}+C\left|v_{n}\right|^{s}|u| Q_{n}+C|u|^{s+1} Q_{n}+C|u|^{s} Q
$$

$\mathrm{e}$

$$
P:=C|u|^{N}+C|u|^{s+1} Q+C|u|^{s} Q
$$

temos que $P_{n}(x) \rightarrow P(x)$ q.t.p. em $\mathbb{R}^{N}$.

De modo análogo à prova do Lema 1.3 , podemos justificar utilizando 2.33 , que existem $\alpha>\alpha_{0}, t>1$ e uma constante $C>0$, tais que $\left(Q_{n}\right)$ e $Q$ pertencem a $L^{t}\left(\mathbb{R}^{N}\right)$ e $\left|Q_{n}\right|_{t} \leq C$. Assim, aplicando o Lema de Brezis-Lieb, podemos concluir que

$$
\int_{\mathbb{R}^{N}} P_{n} d x \rightarrow \int_{\mathbb{R}^{N}} P d x
$$

A prova agora segue aplicando o Teorema da Convergência Dominada Generalizada de Lebesgue (ver Apêndice, Teorema A.2.

Análise de $(b)$. Utilizaremos as mesmas notações do item anterior. Note que pela hipótese $\left(H_{7}\right)$ temos

$$
\begin{aligned}
\left|g\left(v_{n}+u\right)-g\left(v_{n}\right)\right| & =\left|\int_{0}^{1} \frac{d}{d t} g\left(v_{n}+t u\right) d t\right| \leq \int_{0}^{1}\left|g^{\prime}\left(v_{n}+t u\right) u\right| d t \\
& \leq C|u|\left[e^{\alpha\left|w_{n}\right|^{N-1}}-S_{N-2}\left(\alpha, w_{n}\right)\right] \leq C|u| Q_{n} .
\end{aligned}
$$

Deste modo,

$$
\left|g\left(v_{n}+u\right)-g\left(v_{n}\right)-g(u)\right|^{r} \leq C|u|^{r} Q_{n}^{r}+C|g(u)|^{r} .
$$


Considere

$$
\tilde{Q}_{n}:=C|u|^{r} Q_{n}^{r}+C|g(u)|^{r}
$$

e

$$
\tilde{Q}:=C|u|^{r} Q^{r}+C|g(u)|^{r}
$$

Claramente, $\tilde{Q}_{n}(x) \rightarrow \tilde{Q}(x)$ q.t.p. em $\mathbb{R}^{N}$.

Com argumentos análogos aos utilizados na prova do Lema 1.3, provamos que existem $\alpha>\alpha_{0}$ e $r, t>1$ tais que $Q,\left(Q_{n}\right)^{r} \in L^{t}\left(\mathbb{R}^{N}\right)$ e $\left(\left(Q_{n}\right)^{r}\right)$ é limitada em $L^{t}\left(\mathbb{R}^{N}\right)$. Logo, por Brezis-Lieb (ver Lema A.5),

$$
\int_{\mathbb{R}^{N}} \tilde{Q}_{n} d x \rightarrow \int_{\mathbb{R}^{N}} \tilde{Q} d x
$$

Novamente, o Teorema de Lebesgue implica em (b).

Análise de $(c)$. Note que

$$
\begin{aligned}
& \left|\int_{\mathbb{R}^{N}} h(x)\left(v_{n}^{+}\right)^{q} d x-\int_{\mathbb{R}^{N}} h(x) u_{n}^{q} d x+\int_{\mathbb{R}^{N}} h(x) u^{q} d x\right| \leq\left|\int_{\mathbb{R}^{N}} h(x)\left(v_{n}^{+}\right)^{q} d x\right| \\
& +\left|\int_{\mathbb{R}^{N}} h(x) u_{n}^{q} d x-\int_{\mathbb{R}^{N}} h(x) u^{q} d x\right| .
\end{aligned}
$$

Uma vez que $\left(v_{n}^{+}\right)^{q}$ é uma sequência limitada em $L^{\frac{N}{q}}\left(\mathbb{R}^{N}\right)$ e $v_{n}^{+}(x) \rightarrow 0$ q.t.p. em $\mathbb{R}^{N}$, temos que $v_{n}^{+} \rightarrow 0$ em $L^{\frac{N}{q}}\left(\mathbb{R}^{N}\right)$. Sendo $h \in L^{\frac{N}{N-q}}\left(\mathbb{R}^{N}\right)$, concluímos que

$$
\int_{\mathbb{R}^{N}} h(x)\left(v_{n}^{+}\right)^{q} d x \rightarrow 0 .
$$

Analogamente,

$$
\int_{\mathbb{R}^{N}} h(x) u_{n}^{q} d x \rightarrow \int_{\mathbb{R}^{N}} h(x) u^{q} d x
$$

provando $(c)$.

Análise de $(d)$. A prova $(d)$ segue diretamente de $(a),(c)$ e 2.28 . 
Análise de $(e)$. Para justificar $(e)$, escolhemos arbitrariamente $\varphi \in W^{1, N}\left(\mathbb{R}^{N}\right) \backslash\{0\}$ e computamos

$$
\begin{aligned}
\left|\left[I_{\lambda}^{\prime}\left(v_{n}\right)-I_{\lambda}^{\prime}\left(u_{n}\right)+I_{\lambda}^{\prime}(u)\right] \varphi\right| & \leq\left.\left|\int_{\mathbb{R}^{N}}\right| \nabla u_{n}\right|^{N-2} \nabla u_{n} \nabla \varphi d x-\int_{\mathbb{R}^{N}}|\nabla u|^{N-2} \nabla u \nabla \varphi d x \mid \\
& +\left.\left|\int_{\mathbb{R}^{N}}\right| \nabla v_{n}\right|^{N-2} \nabla v_{n} \nabla \varphi d x \mid \\
& +\left.\left|\int_{\mathbb{R}^{N}}\right| u_{n}\right|^{N-2} u_{n} \varphi d x-\int_{\mathbb{R}^{N}}|u|^{N-2} u \varphi d x \mid \\
& +\left.\left|\int_{\mathbb{R}^{N}}\right| v_{n}\right|^{N-2} v_{n} \varphi d x|+| \lambda \int_{\mathbb{R}^{N}} h(x)\left(v_{n}^{+}\right)^{q-1} \varphi d x \mid \\
& +\left|\lambda \int_{\mathbb{R}^{N}} h(x) u_{n}^{q-1} \varphi d x-\lambda \int_{\mathbb{R}^{N}} h(x) u^{q-1} \varphi d x\right| \\
& +\left|\int_{\mathbb{R}^{N}}\left[-g\left(v_{n}\right)+g\left(u_{n}\right)-g(u)\right] \varphi d x\right| .
\end{aligned}
$$

Usando os limites $(i)-(v)$, o item $(b)$ e calculando com idéias similares às apresentadas acima os limites envolvendo $\left(v_{n}\right)$, não é difícil concluir que

$$
\left|\left[I_{\lambda}^{\prime}\left(v_{n}\right)-I_{\lambda}^{\prime}\left(u_{n}\right)+I_{\lambda}^{\prime}(u)\right] \varphi\right| \leq o_{n}(1)|| \varphi\left\|+\left|g\left(v_{n}\right)-g\left(u_{n}\right)+g(u)\right|_{r}|\varphi|_{r^{\prime}} \leq o_{n}(1)\right\| \varphi \| .
$$

De onde segue a prova de $(e)$.

Observe que os limites $(d)$ e $(e)$ implicam em

$$
I_{\lambda}\left(v_{n}\right)=c-I_{\lambda}(u)+o_{n}(1) \text { e } I_{\lambda}^{\prime}\left(v_{n}\right)=o_{n}(1) .
$$

Além disso,

$$
I_{\lambda}\left(v_{n}\right)=I_{\infty}\left(v_{n}\right)+o_{n}(1) .
$$

Aplicando a Desigualdade de Hölder, obtemos

$$
\begin{aligned}
\left|I_{\lambda}^{\prime}\left(v_{n}\right) \varphi-I_{\infty}^{\prime}\left(v_{n}\right) \varphi\right| & =\left|\lambda \int_{\mathbb{R}^{N}} h v_{n}^{q-1} \varphi d x\right| \leq \lambda \int_{\mathbb{R}^{N}}\left|h^{\frac{q-1}{q}} v_{n}^{q-1} h^{\frac{1}{q}} \varphi\right| d x \\
& \leq \lambda C\left(\int_{\mathbb{R}^{N}} h v_{n}^{q} d x\right)^{\frac{q-1}{q}}\|\varphi\| .
\end{aligned}
$$

Assim,

$$
\left\|I_{\lambda}^{\prime}\left(v_{n}\right)-I_{\infty}^{\prime}\left(v_{n}\right)\right\|=o_{n}(1)
$$

De (2.34)-2.36) temos

$$
I_{\infty}\left(v_{n}\right)=c-I_{\lambda}(u)+o_{n}(1) \text { e } I_{\infty}^{\prime}\left(v_{n}\right)=o_{n}(1) .
$$


Agora vamos provar que existe $R>0$ tal que

$$
\liminf _{n \rightarrow+\infty} \sup _{y \in \mathbb{R}^{N}} \int_{B_{R}(y)}\left|v_{n}\right|^{N} d x=0 .
$$

De fato, se 2.38 não ocorre, podemos encontrar $R>0, \beta>0$ e uma sequência $\left(y_{n}\right) \subset \mathbb{R}^{N}$ tais que

$$
\int_{B_{R}\left(y_{n}\right)}\left|v_{n}\right|^{N} d x>\beta
$$

Considere a sequência

$$
\hat{v}_{n}(x):=v_{n}\left(x+y_{n}\right)
$$

Claramente, $\left\|\hat{v}_{n}\right\|=\left\|v_{n}\right\|$, ou seja, $\left(\hat{v}_{n}\right)$ é limitada em $W^{1, N}\left(\mathbb{R}^{N}\right)$. Logo, existe $\hat{v} \in$ $W^{1, N}\left(\mathbb{R}^{N}\right)$ tal que

$$
\hat{v}_{n} \rightarrow \hat{v} \quad \text { em } \quad W^{1, N}\left(\mathbb{R}^{N}\right) .
$$

Note que

$$
\int_{B_{R}(0)}\left|\hat{v}_{n}(z)\right|^{N} d z=\int_{B_{R}(0)}\left|v_{n}\left(z+y_{n}\right)\right|^{N} d z=\int_{B_{R}\left(y_{n}\right)}\left|v_{n}(z)\right|^{N} d z>\beta .
$$

Usando a imersão compacta de Sobolev de $W^{1, N}\left(\mathbb{R}^{N}\right)$ em $L^{N}\left(B_{R}(0)\right)$, concluímos que $\hat{v} \neq 0$. É fácil verificar que

$$
I_{\infty}\left(\hat{v}_{n}\right)=I_{\infty}\left(v_{n}\right) \quad \text { e } \quad I_{\infty}^{\prime}\left(\hat{v}_{n}\right)=I_{\infty}^{\prime}\left(v_{n}\right)
$$

Logo, por 2.37) temos

$$
I_{\infty}\left(\hat{v}_{n}\right)=c-I_{\lambda}(u)+o_{n}(1) \text { e } I_{\infty}^{\prime}\left(\hat{v}_{n}\right)=o_{n}(1)
$$

Portanto, $\left(\hat{v}_{n}\right)$ é uma sequência $(P . S)_{c-I_{\lambda}(u)}$ para o funcional $I_{\infty}$ e consequentemente $I_{\infty}^{\prime}(\hat{v})=0$. Observe que pelo Lema de Fatou

$$
\begin{aligned}
c_{\infty} & =\inf _{v \in \mathcal{M}_{\infty}} I_{\infty}(v) \leq I_{\infty}(\hat{v})=I_{\infty}(\hat{v})-\frac{1}{N} I_{\infty}^{\prime}(\hat{v}) \hat{v}=\frac{1}{N} \int_{\mathbb{R}^{N}}[g(\hat{v}) \hat{v}-N G(\hat{v})] d x \\
& \leq \liminf _{n \rightarrow+\infty} \frac{1}{N} \int_{\mathbb{R}^{N}}\left[g\left(\hat{v}_{n}\right) \hat{v}_{n}-N G\left(\hat{v}_{n}\right)\right] d x=\liminf _{n \rightarrow+\infty} I_{\infty}\left(\hat{v}_{n}\right) \\
& =c-I_{\lambda}(u) \leq c+\mathrm{K} \lambda^{\theta},
\end{aligned}
$$

onde na última desigualdade, aplicamos o Lema 2.11. Logo, $c_{\infty}-\mathrm{K} \lambda^{\theta} \leq c$, o que contradiz a hipótese. Desta forma, existe $R>0$ tal que

$$
\liminf _{n \rightarrow+\infty} \sup _{y \in \mathbb{R}^{N}} \int_{B_{R}(y)}\left|v_{n}\right|^{N} d x=0
$$


o que implica que $v_{n} \rightarrow 0$ em $L^{t}\left(\mathbb{R}^{N}\right)$, para todo $t>N$ (ver Apêndice, Lema A.4). Usando este fato, podemos provar que

$$
\lim _{n \rightarrow+\infty} \int_{\mathbb{R}^{N}} g\left(v_{n}\right) v_{n} d x=0
$$

De fato, para $\epsilon>0, s>N$ e $\alpha>\alpha_{0}$ temos

$$
\begin{aligned}
\int_{\mathbb{R}^{N}}\left|g\left(v_{n}\right) v_{n}\right| d x & \leq \epsilon\left|v_{n}\right|_{N}^{N}+C_{\epsilon} \int_{\mathbb{R}^{N}}\left|v_{n}\right|^{s}\left[e^{\alpha\left|v_{n}\right|^{\frac{N}{N-1}}}-S_{N-2}\left(\alpha, v_{n}\right)\right] d x \\
& \leq\left.\epsilon C|| v_{n}\right|^{N}+C_{\epsilon}\left|v_{n}\right|_{s t^{\prime}}^{s}\left|e^{\alpha\left|v_{n}\right|^{\frac{N}{N-1}}}-S_{N-2}\left(\alpha, v_{n}\right)\right|_{t}
\end{aligned}
$$

onde escolhemos $\alpha>\alpha_{0}$ e $t>1(t \approx 1)$, de modo que

$$
\left|e^{\alpha\left|v_{n}\right|^{\frac{N}{N-1}}}-S_{N-2}\left(\alpha, v_{n}\right)\right|_{t} \leq C
$$

para alguma constante $C>0$ (ver Lema 1.3 ). Portanto,

$$
\int_{\mathbb{R}^{N}}\left|g\left(v_{n}\right) v_{n}\right| d x \leq \epsilon C|| v_{n}||^{N}+C_{\epsilon}\left|v_{n}\right|_{s t^{\prime}}^{s}
$$

ou ainda,

$$
\int_{\mathbb{R}^{N}}\left|g\left(v_{n}\right) v_{n}\right| d x \leq \epsilon C_{1}+o_{n}(1)
$$

provando 2.42. Neste caso,

$$
\left\|v_{n}\right\|^{N}=I_{\lambda}^{\prime}\left(v_{n}\right) v_{n}+\lambda \int_{\mathbb{R}^{N}} h(x)\left(v_{n}^{+}\right)^{q} d x+\int_{\mathbb{R}^{N}} g\left(v_{n}\right) v_{n} d x=o_{n}(1) .
$$

Ou seja, $u_{n} \rightarrow u$ em $W^{1, N}\left(\mathbb{R}^{N}\right)$, como queríamos demonstrar.

Uma vez que para $\lambda<\lambda_{1}$, o funcional $I_{\lambda}$ satisfaz a geometria do Passo da Montanha (ver Lema 2.10), existe uma sequência $\left(u_{n}\right) \subset W^{1, N}\left(\mathbb{R}^{N}\right)$ satisfazendo

$$
I_{\lambda}\left(u_{n}\right) \rightarrow c \quad \text { e } \quad I_{\lambda}^{\prime}\left(u_{n}\right) \rightarrow 0, \quad \text { quando } n \rightarrow+\infty
$$

onde

$$
c=\inf _{\gamma \in \Gamma} \max _{t \in[0,1]} I_{\lambda}(\gamma(t))
$$

com

$$
\Gamma=\left\{\gamma \in C\left([0,1], W^{1, N}\left(\mathbb{R}^{N}\right)\right) ; \gamma(0)=0 \text { e } \gamma(1)=e\right\},
$$

onde $e$ é dado pelo Lema 2.10. 
Proposição 2.4 Existe $\lambda_{2}>0$ tal que, para $0<\lambda<\lambda_{2}$, o nível c satisfaz

$$
c<c_{\infty}-K \lambda^{\theta}
$$

onde K é a constante dada no Lema 2.11.

Demonstração. Considere $\Psi \in W^{1, N}\left(\mathbb{R}^{N}\right)$ tal que $c_{\infty}=I_{\infty}(\Psi)$ e $I_{\infty}^{\prime}(\Psi)=0$. Fixe $\delta_{1}:=\left(c_{\infty} / 2 \mathrm{~K}\right)^{1 / \theta}>0$ e observe que

$$
c_{\infty}-\mathrm{K} \lambda^{\theta}>\frac{c_{\infty}}{2}, \quad \forall \lambda<\delta_{1} .
$$

Uma vez que $I_{\lambda}(t \Psi) \rightarrow 0$, quando $t \rightarrow 0$, podemos fixar $t_{0}>0$ suficientemente pequeno tal que

$$
\sup _{0 \leq t \leq t_{0}} I_{\lambda}(t \Psi)<\frac{c_{\infty}}{2}<c_{\infty}-\mathrm{K} \lambda^{\theta}, \quad \forall \lambda<\delta_{1}
$$

Por outro lado,

$$
\begin{aligned}
I_{\lambda}(t \Psi) & =I_{\infty}(t \Psi)-\lambda \frac{t^{q}}{q} \int_{\mathbb{R}^{N}} h(x) \Psi^{q} d x \leq \max _{t \geq 0} I_{\infty}(t \Psi)-\lambda \frac{t^{q}}{q} \int_{\mathbb{R}^{N}} h(x) \Psi^{q} d x \\
& \leq I_{\infty}(\Psi)-\lambda \frac{t^{q}}{q} \int_{\mathbb{R}^{N}} h(x) \Psi^{q} d x=c_{\infty}-\lambda \frac{t^{q}}{q} \int_{\mathbb{R}^{N}} h(x) \Psi^{q} d x .
\end{aligned}
$$

Portanto,

$$
\sup _{t \geq t_{0}} I_{\lambda}(t \Psi) \leq c_{\infty}-\lambda \frac{t_{0}^{q}}{q} \int_{\mathbb{R}^{N}} h(x) \Psi^{q} d x .
$$

Fixe

$$
\delta_{2}=\left(\frac{t_{0}^{q}}{\mathrm{~K} q} \int_{\mathbb{R}^{N}} h(x) \Psi^{q} d x\right)^{\frac{N-q}{q}}
$$

Neste caso,

$$
-\lambda \frac{t_{0}^{q}}{q} \int_{\mathbb{R}^{N}} h(x) \Psi^{q} d x<-\mathrm{K} \lambda^{\theta}, \quad \forall \lambda<\delta_{2}
$$

de onde segue que

$$
\sup _{t \geq t_{0}} I_{\lambda}(t \Psi)<c_{\infty}-\mathrm{K} \lambda^{\theta}, \quad \forall \lambda<\delta_{2} .
$$

Escolhendo $\lambda_{2}=\min \left\{\delta_{1}, \delta_{2}\right\}$ e usando 2.48 e 2.49, temos

$$
\sup _{t \geq 0} I_{\lambda}(t \Psi)<c_{\infty}-\mathrm{K} \lambda^{\theta}, \quad \forall \lambda<\lambda_{2}
$$

consequentemente,

$$
0<c<c_{\infty}-\mathrm{K} \lambda^{\theta}, \quad \forall \lambda<\lambda_{2}
$$


Proposição 2.5 Suponha que g satisfaz $\left(H_{c}\right),\left(H_{0}\right),\left(H_{4}\right),\left(H_{5}\right),\left(H_{6}\right)$ e $\left(H_{7}\right)$. Então, existe uma constante $\Lambda_{1}>0$ tal que, para $0<\lambda<\Lambda_{1}$, o problema $\left(P_{3}\right)$ possui uma solução $u_{1}$ verificando $I_{\lambda}\left(u_{1}\right)>0$.

Demonstração. Seja $\Lambda_{1}<\min \left\{\lambda_{1}, \lambda_{2}\right\}$, onde $\lambda_{1}$ e $\lambda_{2}$ são obtidos no Lema 2.10 e na Proposição 2.4, respectivamente. Considerando a sequência $\left(u_{n}\right)$ dada em (2.47), segue das Proposições 2.3 e 2.4 que o funcional $I_{\lambda}$ verifica a condição $(P . S)_{c}$, para $0<\lambda<\Lambda_{1}$. Assim, a menos de subsequência, temos que $\left(u_{n}\right)$ converge fortemente para uma função $u_{1} \in W^{1, N}\left(\mathbb{R}^{N}\right)$. Além disso,

$$
I_{\lambda}^{\prime}\left(u_{n}\right) \rightarrow I_{\lambda}^{\prime}\left(u_{1}\right)=0, \text { quando } n \rightarrow+\infty
$$

e

$$
I_{\lambda}\left(u_{n}\right) \rightarrow I_{\lambda}\left(u_{1}\right)=c>0 \text { quando } n \rightarrow+\infty .
$$

Logo, o problema possui uma solução fraca $u_{1} \operatorname{com} I_{\lambda}\left(u_{1}\right)>0$.

\subsubsection{Segunda solução}

Proposição 2.6 Suponha que g satisfaz $\left(H_{c}\right),\left(H_{0}\right),\left(H_{4}\right),\left(H_{5}\right),\left(H_{6}\right)$ e $\left(H_{7}\right)$. Então, existe uma constante $\Lambda_{2}>0$ tal que, para $0<\lambda<\Lambda_{2}$, o problema $\left(P_{3}\right)$ possui uma solução $u_{2}$ verificando $I_{\lambda}\left(u_{2}\right)<0$.

Demonstração. Seja $\bar{B}_{\rho}(0):=\left\{u \in W^{1, N}\left(\mathbb{R}^{N}\right) ;\|u\| \leq \rho\right\}$ o espaço métrico completo munido com a métrica dada por $d(u, v)=\|u-v\|$. Uma vez que o funcional $I_{\lambda}$ é de classe $C^{1}$ e limitado inferiormente em $\bar{B}_{\rho}(0)$, para $\lambda<\lambda_{1}$ (ver Lema 2.10), podemos aplicar o Princípio Variacional de Ekeland (ver Apêndice, Teorema A.7) para obter uma sequência $\left(u_{n}\right) \subset \bar{B}_{\rho}(0)$ verificando

$$
I_{\lambda}\left(u_{n}\right) \longrightarrow d:=\inf \left\{I_{\lambda}(u) ; u \in \bar{B}_{\rho}(0)\right\}
$$

$\mathrm{e}$

$$
\left\|I_{\lambda}^{\prime}\left(u_{n}\right)\right\| \longrightarrow 0, \quad \text { quando } n \rightarrow+\infty,
$$

onde o último limite é obtido de modo análogo a (2.17). Além disso, para toda $v \in$ $W^{1, N}\left(\mathbb{R}^{N}\right)$ temos

$$
I_{\lambda}(t v) \leq \frac{t^{N}}{N}\|v\|^{N}-\frac{\lambda t^{q}}{q} \int_{\mathbb{R}^{N}} h(x) v_{+}^{q} d x<0
$$


para $t>0$ próximo de 0 , consequentemente, $d<0$. Portanto, $\left(u_{n}\right) \subset \bar{B}_{\rho}(0)$ é uma sequência $(P . S)_{d}$ para o funcional $I_{\lambda} \operatorname{com} d<0$.

Afirmamos que

$$
\limsup _{n \rightarrow+\infty}\left\|u_{n}\right\|^{N}<\left(\frac{\alpha_{N}}{\alpha_{0}}\right)^{N-1}
$$

De fato,

$$
I_{\lambda}\left(u_{n}\right)-\frac{1}{\nu} I_{\lambda}^{\prime}\left(u_{n}\right)\left(u_{n}\right) \leq d+o_{n}(1)+o_{n}(1)\left\|u_{n}\right\| .
$$

Por outro lado, por $\left(H_{4}\right)$

$$
\begin{aligned}
I_{\lambda}\left(u_{n}\right)-\frac{1}{\nu} I_{\lambda}^{\prime}\left(u_{n}\right)\left(u_{n}\right) & =\left(\frac{1}{N}-\frac{1}{\nu}\right)\left\|u_{n}\right\|^{N}-\lambda\left(\frac{1}{q}-\frac{1}{\nu}\right) \int_{\mathbb{R}^{N}} h(x) u_{n}^{q} d x \\
& -\int_{\mathbb{R}^{N}}\left(G\left(u_{n}\right)-\frac{1}{\nu} g\left(u_{n}\right) u_{n}\right) d x \\
& \geq\left(\frac{1}{N}-\frac{1}{\nu}\right)\left\|u_{n}\right\|^{N}-\lambda\left(\frac{1}{q}-\frac{1}{\nu}\right) C|h|_{\theta}\left\|u_{n}\right\|_{q}^{q} .
\end{aligned}
$$

As desigualdades 2.50) e 2.51) resultam em

$$
\left(\frac{1}{N}-\frac{1}{\nu}\right)\left\|u_{n}\right\|^{N} \leq \lambda\left(\frac{1}{q}-\frac{1}{\nu}\right) C|h|_{\theta}\left\|u_{n}\right\|^{q}+d+o_{n}(1)\left\|u_{n}\right\|+o_{n}(1) .
$$

Logo, com o mesmo raciocínio usado para obter a estimativa 2.12 obtemos

$$
\limsup _{n \rightarrow \infty}\left\|u_{n}\right\|^{N} \leq\left(\lambda \frac{\left(\frac{1}{q}-\frac{1}{\nu}\right) C|h|_{\theta}}{\left(\frac{1}{N}-\frac{1}{\nu}\right)}\right)^{\frac{N}{N-q}} .
$$

Escolhendo $\lambda_{2}:=\left(\frac{q(\nu-N)}{2 C|h|_{\theta}(\nu-q)}\right)\left(\left(\frac{\alpha_{N}}{\alpha_{0}}\right)^{N-1}\right)^{\frac{N-q}{N}}$, temos

$$
\limsup _{n \rightarrow \infty}\left\|u_{n}\right\|^{N}<\left(\frac{\alpha_{N}}{\alpha_{0}}\right)^{N-1}, \quad \text { para } \lambda<\lambda_{2}
$$

Além disso, sendo a sequência $\left(u_{n}\right)$ limitada em $W^{1, N}\left(\mathbb{R}^{N}\right)$, existe $u_{2} \in W^{1, N}\left(\mathbb{R}^{N}\right)$ satisfazendo

$$
\begin{gathered}
u_{n} \rightarrow u_{2} \text { em } W^{1, N}\left(\mathbb{R}^{N}\right), \\
u_{n}(x) \rightarrow u_{2}(x) \text { q.t.p. em } \mathbb{R}^{N}
\end{gathered}
$$

e

$$
u_{n} \rightarrow u_{2} \text { em } L_{l o c}^{t}\left(\mathbb{R}^{N}\right), \quad \text { para } t \geq N
$$

Considerando $\varphi \in C_{0}^{\infty}\left(\mathbb{R}^{N}\right)$ e procedendo de maneira análoga à prova do Lema 1.8 (ver também Lema 2.6), podemos justificar as seguintes afirmações: 
(i) $\nabla u_{n}(x) \rightarrow \nabla u_{2}(x)$ q.t.p. em $\mathbb{R}^{N}$;

(ii) $\int_{\mathbb{R}^{N}}\left(g\left(u_{n}\right) \varphi-g\left(u_{2}\right) \varphi\right) d x=o_{n}(1) ;$

(iii) $\int_{\mathbb{R}^{N}}\left(h(x) u_{n}^{q-1} \varphi-h(x) u_{2}^{q-1} \varphi\right) d x=o_{n}(1) ;$

(iv) $\int_{\mathbb{R}^{N}}\left(\left|\nabla u_{n}\right|^{N-2} \nabla u_{n} \nabla \varphi-\left|\nabla u_{2}\right|^{N-2} \nabla u_{2} \nabla \varphi\right) d x=o_{n}(1)$

(v) $\int_{\mathbb{R}^{N}}\left(\left|u_{n}\right|^{N-2} u_{n} \varphi-\left|u_{2}\right|^{N-2} u_{2} \varphi\right) d x=o_{n}(1)$.

Consequentemente, $I_{\lambda}^{\prime}\left(u_{n}\right) \varphi=I_{\lambda}^{\prime}\left(u_{2}\right) \varphi+o_{n}(1)$, o que nos leva a concluir que $I_{\lambda}^{\prime}\left(u_{2}\right)=0$. Portanto, $u_{2}$ é uma solução do problema $\left(P_{3}\right)$.

Resta apenas mostrar que $I_{\lambda}\left(u_{2}\right)<0$. Para isto, observe que

$$
\begin{aligned}
d+o_{n}(1) & =I_{\lambda}\left(u_{n}\right)-\frac{1}{\nu} I_{\lambda}^{\prime}\left(u_{n}\right) u_{n}=\left(\frac{1}{N}-\frac{1}{\nu}\right)\left\|u_{n}\right\|^{N} \\
& -\lambda\left(\frac{1}{q}-\frac{1}{\nu}\right) \int_{\mathbb{R}^{N}} h(x) u_{2}^{q} d x-\int_{\mathbb{R}^{N}}\left[G\left(u_{n}\right)-\frac{1}{\nu} g\left(u_{n}\right) u_{n}\right] d x+o_{n}(1) .
\end{aligned}
$$

Como consequência do Lema de Fatou, segue que

$$
d=\lim _{n \rightarrow \infty}\left(I_{\lambda}\left(u_{n}\right)-\frac{1}{\nu} I_{\lambda}^{\prime}\left(u_{n}\right) u_{n}\right) \geq I_{\lambda}\left(u_{2}\right)-\frac{1}{\nu} I_{\lambda}^{\prime}\left(u_{2}\right) u_{2}=I_{\lambda}\left(u_{2}\right) .
$$

Assim, $I_{\lambda}\left(u_{2}\right) \leq d<0$. Escolhendo $\Lambda_{2}=\min \left\{\lambda_{1}, \lambda_{2}\right\}$ concluímos a prova da proposição.

\section{Demonstração do Teorema 3}

Segue diretamente das Proposições 2.5 e 2.6.

\subsubsection{Multiplicidade de soluções com energia negativa}

\section{Demonstração do Teorema 5}

Associamos ao problema $\left(P_{3}\right)$ o seguinte funcional energia

$$
I_{\lambda}(u)=\frac{1}{N}\|u\|^{N}-\frac{\lambda}{q} \int_{\mathbb{R}^{N}} h(x)|u|^{q} d x-\int_{\mathbb{R}^{N}} G(u) d x .
$$

Iremos supor que $g$ é uma função ímpar, portanto, $I_{\lambda}$ é um funcional par. Vamos utilizar as mesmas idéias apresentadas na Subseção 2.1.3. Inicialmente observamos que de modo similar a 2.20 podemos deduzir

$$
I_{\lambda}(u) \geq \frac{1}{2 N}\|u\|^{N}-\frac{C_{1}|h|_{\theta}}{q} \lambda\|u\|^{q}-C_{3}\|u\|^{s}, \quad \forall u \in W^{1, N}\left(\mathbb{R}^{N}\right),\|u\| \leq R,
$$


onde $R>0$ verifica $\alpha R^{\frac{N}{N-1}}<\alpha_{N}$. Fixamos $0<R_{1}<R$ suficientemente pequeno tal que

$$
\frac{1}{4 N} R_{1}^{N}-C_{3} R_{1}^{s}>0
$$

Escolha $\lambda<\lambda_{1}:=\frac{q}{4 N C_{1}|h|_{\theta}} R_{1}^{N-q}$ e defina

$$
\kappa(x)=\frac{1}{2 N} x^{N}-\frac{C_{1}|h|_{\theta}}{q} \lambda_{1} x^{q}-C_{3} x^{s}, \quad \forall x \geq 0 .
$$

Observe que

$$
I_{\lambda}(u) \geq \kappa(\|u\|), \text { para }\|u\|<R_{1} \text { e } \forall \lambda<\lambda_{1} .
$$

Seja $R_{0}:=\max \left\{0<x \leq R_{1} ; \kappa(x) \leq 0\right\}$ e considere uma função $\tau:[0,+\infty) \rightarrow[0,1]$, de classe $C^{\infty}$, verificando

$$
\begin{gathered}
\tau(x)=1, \text { se } x \leq R_{0}, \\
\tau(x)=0, \text { se } x \geq R_{1} .
\end{gathered}
$$

Defina

$$
\tilde{\kappa}(x)=\frac{1}{2 N} x^{N}-\frac{C_{1}|h|_{\theta}}{q} \lambda_{1} x^{q}-C_{3} x^{s} \tau(x)
$$

e

$$
\tilde{I}_{\lambda}(u)=\frac{1}{N}\|u\|^{N}-\frac{\lambda}{q} \int_{\mathbb{R}^{N}} h(x) u_{+}^{q} d x-\tau(u) \int_{\Omega} G(u) d x,
$$

onde $\tau(u)=\tau(\|u\|)$.

As seguintes afirmações são verdadeiras:

(i) $\tilde{I}_{\lambda} \in C^{1}\left(W^{1, N}\left(\mathbb{R}^{N}\right), \mathbb{R}\right)$;

(ii) se $\tilde{I}_{\lambda}(u)<0$, então $\|u\|<R_{0}$;

(iii) $I_{\lambda}(v)=\tilde{I}_{\lambda}(v)$, para cada $v$ suficientemente próximo de $u$ com $\|u\|<R_{0}$;

(iv) $\tilde{I}_{\lambda}$ satisfaz a condição $(P . S)_{d} \operatorname{com} d<0$.

Para justificar (iv) aplicamos a Proposição 2.3. Repetindo a prova do Lema 2.9 e a demonstração do Teorema 4, concluímos que existe uma quantidade infinita de pontos críticos de $\tilde{I}_{\lambda}$ com energia negativa. Logo, pelas afirmações acima tais pontos são pontos críticos de $I_{\lambda}$. Portanto, garantimos a existência de uma quantidade infinita de soluções para o problema $\left(P_{3}\right)$. 


\title{
CAPÍTULO 3
}

\section{MULTIPLICIDADE DE SOLUÇÕES VIA TEORIA DO}

\author{
GÊNERO E UM PROBLEMA COM QUEBRA DE SIMETRIA
}

\subsection{Soluções para uma classe de equações elípticas em}

\section{domínio limitado}

Nesta seção, estudamos a seguinte classe de problemas elípticos

$$
\left\{\begin{array}{l}
-\Delta_{N} u=a(x)|u|^{N-2} u+\lambda f(x, u)+g(x, u), \quad x \in \Omega, \\
u=0, \quad x \in \partial \Omega
\end{array}\right.
$$

onde $\Omega \subset \mathbb{R}^{N}$ é um domínio limitado com fronteira suave, $N \geq 2, a \in L^{\infty}(\Omega)$ e $\lambda$ é um parâmetro positivo.

Vamos assumir o seguinte conjunto de hipóteses sobre a função $f$ :

$\left(\hat{\mathbf{H}}_{\mathbf{0}}\right) f \in C(\Omega \times \mathbb{R}, \mathbb{R})$ e satisfaz

$$
f(x, s)=o\left(|s|^{N-1}\right), \text { quando } s \rightarrow 0,
$$

uniformemente para $x \in \Omega$; 
$\left(\hat{\mathbf{H}}_{\mathbf{c}}\right)$ existe $\alpha_{0}>0$ tal que

$$
\lim _{|s| \rightarrow \infty} \frac{|f(x, s)|}{e^{\alpha|s|^{N-1}}}=\left\{\begin{array}{lll}
0 & \text { se } & \alpha>\alpha_{0} \\
+\infty & \text { se } & \alpha<\alpha_{0}
\end{array}\right.
$$

uniformemente para $x \in \Omega$;

$\left(\hat{\mathbf{H}}_{4}\right)$ existe $\nu>N$ tal que

$$
0<\nu F(x, s) \leq f(x, s) s, \quad \forall|s|>0 \quad \text { e } \quad \forall x \in \Omega
$$

$\left(\hat{\mathbf{H}}_{\mathbf{5}}\right)$ existem $p>N$ e uma constante $C_{p}>0$ tais que

$$
f(x, s) \geq C_{p} s^{p-1}, \quad \forall s \geq 0 \quad \text { e } \quad \forall x \in \Omega
$$

Para a função $g$ admitimos, além das hipóteses $\left(\hat{H}_{0}\right),\left(\hat{H}_{4}\right)$ e $\left(\hat{H}_{5}\right)$, a seguinte condição de crescimento subcrítico exponencial:

$\left(\hat{\mathbf{H}}_{\mathbf{s c}}\right)$ dado $\alpha>0$, existe $C_{\alpha}>0$ tal que

$$
|g(x, s)| \leq C_{\alpha} e^{\alpha|s|^{\frac{N}{N-1}}}, \forall s \in \mathbb{R} \quad \text { e } \quad \forall x \in \Omega .
$$

Para finalizar as hipóteses, iremos supor que

$\left(\hat{\mathbf{H}}_{\mathbf{a}}\right)$ existe $A>0$ tal que

$$
\int_{\Omega}\left(|\nabla u|^{N}-a(x)|u|^{N}\right) d x \geq A \int_{\Omega}|u|^{N} d x, \quad \forall u \in W_{0}^{1, N}(\Omega) .
$$

Nosso objetivo é estudar a existência de múltiplas soluções para o problema $\left(P_{4}\right)$, o que se reduz a provar a multiplicidade de pontos críticos para o funcional $I_{\lambda}: W_{0}^{1, N}(\Omega) \rightarrow \mathbb{R}$ dado por

$$
I_{\lambda}(u)=\frac{1}{N} \int_{\Omega}\left[|\nabla u|^{N}-a(x)|u|^{N}\right] d x-\lambda \int_{\Omega} F(x, u) d x-\int_{\Omega} G(x, u) d x .
$$

Como sabemos, $I_{\lambda} \in C^{1}\left(W_{0}^{1, N}(\Omega), \mathbb{R}\right)$ (ver Observação 1.2 e

$$
I_{\lambda}^{\prime}(u) v=\int_{\Omega}\left[|\nabla u|^{N-2} \nabla u \nabla v-a(x)|u|^{N-2} u v\right] d x-\lambda \int_{\Omega} f(x, u) v d x-\int_{\Omega} g(x, u) v d x,
$$

$\forall u, v \in W_{0}^{1, N}(\Omega)$. 
Nesta seção, assumimos que $f$ e $g$ são funções ímpares com respeito a $u$, portanto, $I_{\lambda}$ é um funcional par. Logo, se $u$ é ponto crítico de $I_{\lambda},-u$ também será.

No espaço $W_{0}^{1, N}(\Omega)$ consideramos a norma dada por

$$
\|u\|=\left(\int_{\Omega}|\nabla u|^{N} d x\right)^{\frac{1}{N}}, \quad u \in W_{0}^{1, N}(\Omega) .
$$

Considere a função $\|\cdot\|_{*}$ que associa a cada $u \in W_{0}^{1, N}(\Omega)$ o número

$$
\|u\|_{*}=\left(\int_{\Omega}\left(|\nabla u|^{N}-a(x)|u|^{N}\right) d x\right)^{1 / N} .
$$

$\mathrm{Na}$ afirmação a seguir, provamos uma importante relação entre as funções $\|$.$\| e \|.\|_{*}$.

Afirmação 3.1 Existem constantes positivas $\theta$ e $\mu$ verificando

$$
\theta\|u\|^{N} \leq\|u\|_{*}^{N} \leq \mu\|u\|^{N}, \quad \forall u \in W_{0}^{1, N}(\Omega)
$$

Prova. Utilizando a imersão de Sobolev $W_{0}^{1, N}(\Omega) \hookrightarrow L^{N}(\Omega)$, temos

$$
\|u\|_{*}^{N} \leq\|u\|^{N}+|a|_{\infty}|u|_{N}^{N} \leq\left(1+|a|_{\infty} C\right)\|u\|^{N}=\mu\|u\|^{N}
$$

onde $\mu:=1+|a|_{\infty} C$. Por outro lado, fixando uma constante $0<C_{0}<\frac{A}{|a|_{\infty}}$, deduzimos que

$$
\begin{aligned}
\|u\|_{*}^{N} & =\frac{1+C_{0}}{1+C_{0}} \int_{\Omega}\left(|\nabla u|^{N}-a(x)|u|^{N}\right) d x \\
& =\frac{1}{1+C_{0}} \int_{\Omega}\left(|\nabla u|^{N}-a(x)|u|^{N}\right) d x+\frac{C_{0}}{1+C_{0}} \int_{\Omega}|\nabla u|^{N} d x-\frac{C_{0}}{1+C_{0}} \int_{\Omega} a(x)|u|^{N} d x .
\end{aligned}
$$

Pela hipótese $\left(H_{a}\right)$,

$$
\|u\|_{*}^{N} \geq \frac{A}{1+C_{0}}|u|_{N}^{N}+\frac{C_{0}}{1+C_{0}}\|u\|^{N}-\frac{C_{0}}{1+C_{0}}|a|_{\infty}|u|_{N}^{N}=\frac{A-C_{0}|a|_{\infty}}{1+C_{0}}|u|_{N}^{N}+\frac{C_{0}}{1+C_{0}}\|u\|^{N} .
$$

Uma vez que $A-C_{0}|a|_{\infty}>0$, conclui-se que

$$
\|u\|_{*}^{N} \geq \frac{C_{0}}{1+C_{0}}\|u\|^{N}=\theta\|u\|^{N}
$$

para $\theta:=C_{0} /\left(1+C_{0}\right)$. De 3.2 e 3.3 , obtemos

$$
\theta\|u\|^{N} \leq\|u\|_{*}^{N} \leq \mu\|u\|^{N},
$$

o que prova a afirmação. 


\subsubsection{Teorema abstrato}

Apresentamos nesta seção, uma versão do Teorema do Passo da Montanha de Ambrosetti-Rabinowitz (ver [66], pág. 55). No entanto, observamos que podemos adaptar o referido resultado ao caso em que o funcional satisfaz a condição Palais-Smale abaixo de um determinado valor. Como dito na Introdução, faremos a demonstração com as devidas adaptações, para uma melhor compreensão deste texto.

Seja $E=V \oplus X$ um espaço de Banach de dimensão infinita com $\operatorname{dim} V=k<+\infty$ e considere $I$ um funcional de classe $C^{1}(E, \mathbb{R})$. Vamos assumir que o funcional $I$ satisfaz as condições abaixo:

$\left(I_{1}\right) I(0)=0$ e $I$ é par;

$\left(I_{2}\right)$ existem $\beta, \rho>0$ tais que $I(u) \geq \beta>0$, para cada $u \in \partial B_{\rho} \cap X$;

$\left(I_{3}\right)$ existe $\mathcal{S}>0$ tal que $I$ satisfaz a condição $(P . S)_{c}$, para $0<c<\mathcal{S}$;

$\left(I_{4}\right)$ para cada $\tilde{E} \subset E$, subespaço vetorial de dimensão finita, existe $R=R(\tilde{E})$ tal que

$$
I(u) \leq 0, \quad \forall u \in \tilde{E} \backslash B_{R}(0)
$$

Seja $\left\{e_{1}, e_{2}, e_{3}, \ldots, e_{k}\right\}$ uma base do espaço $V$. Para cada $m \geq k$, escolha indutivamente $e_{m+1} \notin E_{m}:=\operatorname{span}\left\{e_{1}, e_{2}, e_{3}, \ldots, e_{m}\right\}$. Considere $R_{m}=R\left(E_{m}\right)$, dado por $\left(I_{4}\right)$, e defina os seguintes conjuntos

$$
\begin{gathered}
D_{m}:=B_{R_{m}} \cap E_{m} \\
G_{m}:=\left\{h \in C\left(D_{m}, E\right) ; h \text { é ímpar e } h(u)=u, \forall u \in \partial B_{R_{m}} \cap E_{m}\right\}
\end{gathered}
$$

e

$$
\Gamma_{j}:=\left\{h\left(\overline{D_{m} \backslash Y}\right) ; h \in G_{m}, m \geq j, Y \in \Sigma \text { e } \gamma(Y) \leq m-j\right\}
$$

onde estamos denotando por $\gamma(Y)$, o gênero do conjunto $Y \in \Sigma$ (ver Apêndice), com

$$
\Sigma:=\{Y \subset E \backslash\{0\} ; Y \text { é fechado em } E \text { e } Y=-Y\}
$$

Observe que $\Gamma_{j} \neq \emptyset$, pois a aplicação identidade $i_{d} \in G_{m}, \forall m \in \mathbb{N}$. Iremos agora justificar as seguintes propriedades envolvendo os conjuntos $\Gamma_{j}$ :

(i) $\Gamma_{j+1} \subset \Gamma_{j}$, para todo $j \in \mathbb{N}$;

(ii) Se $B \in \Gamma_{j}$ e $F \in \Sigma \operatorname{com} \gamma(F) \leq r<j$, então $\overline{B \backslash F} \in \Gamma_{j-r}$. 
Justificativa de $(i)$. Escolhemos arbitrariamente $B=h\left(\overline{D_{m} \backslash Y}\right) \in \Gamma_{j+1}$. Então, pela definição de $\Gamma_{j+1}$,

$$
m \geq j+1 \geq j, \quad h \in G_{m} \text { e } Y \in \Sigma
$$

com

$$
\gamma(Y) \leq m-(j+1) \leq m-j
$$

Assim, $B \in \Gamma_{j}$, provando $(i)$.

Justificativa de $(i i)$. Seja $B=h\left(\overline{D_{m} \backslash Y}\right) \in \Gamma_{j}$ e $F \in \Sigma$ tal que $\gamma(F) \leq r<j$. Afirmamos que

$$
\overline{B \backslash F}=h\left(\overline{D_{m} \backslash\left(Y \cup h^{-1}(F)\right)}\right) .
$$

Assumimos, por um momento, esta afirmação. Observe que $h^{-1}(F) \in \Sigma$ pois $h$ é contínua e ímpar. Neste caso, $Y \cup h^{-1}(F) \in \Sigma$ e as propriedades de gênero asseguram que valem as desigualdades abaixo

$$
\gamma\left(Y \cup h^{-1}(F)\right) \leq \gamma(Y)+\gamma\left(h^{-1}(F)\right) \leq \gamma(Y)+\gamma(F) \leq m-j+r
$$

ou seja,

$$
\gamma\left(Y \cup h^{-1}(F)\right) \leq m-(j-r)
$$

Logo, 3.4 e 3.5 implicam que $\overline{B \backslash F} \in \Gamma_{j-r}$.

Nos resta agora provar a igualdade (3.4). Para isto, fixamos $b \in B \backslash F$, o que significa que $b=h(w)$, onde $w \in \overline{D_{m} \backslash Y}$ e $h(w) \notin F$. Logo,

$$
w \in\left(\overline{D_{m} \backslash Y}\right) \backslash h^{-1}(F) \subset \overline{D_{m} \backslash\left(Y \cup h^{-1}(F)\right)},
$$

ou ainda,

$$
b \in h\left(\overline{D_{m} \backslash\left(Y \cup h^{-1}(F)\right)}\right)
$$

Portanto,

$$
\overline{B \backslash F} \subset h\left(\overline{D_{m} \backslash\left(Y \cup h^{-1}(F)\right)}\right)
$$

Por outro lado, seja

$$
b \in h\left(D_{m} \backslash\left(Y \cup h^{-1}(F)\right)\right) .
$$

Assim, $b=h(w)$, onde $w \in D_{m}, w \notin Y$ e $w \notin h^{-1}(F)$. Desta forma, $b \in h\left(D_{m} \backslash Y\right) \backslash F$, o que implica que $b \in \overline{B \backslash F}$. Logo, podemos concluir que

$$
h\left(\overline{D_{m} \backslash\left(Y \cup h^{-1}(F)\right)}\right) \subset \overline{B \backslash F} .
$$

De (3.6) e (3.7) provamos (3.4) e concluímos a prova de $(i i)$. 
Definimos agora o seguinte nível minimax

$$
c_{j}:=\inf _{K \in \Gamma_{j}} \sup _{u \in K} I(u)
$$

Observe que $c_{j}<\infty$, para cada $j \in \mathbb{N}$. De fato, considere $\bar{E}$ um subespaço de $E$ com $\operatorname{dim} \bar{E}=j$ e seja $K_{R}=\bar{E} \cap B_{R}(0) \in \Gamma_{j}$. Segue da definição de $c_{j}$ que

$$
c_{j} \leq \sup _{u \in K_{R}} I(u)<\infty
$$

Finalmente, defina os seguintes conjuntos

$$
K_{c}:=\left\{u \in E ; I^{\prime}(u)=0 \text { e } I(u)=c\right\}
$$

e

$$
A_{c}:=\{u \in E ; I(u) \leq c\}
$$

Teorema 3.1 Se I satisfaz as hipóteses $\left(I_{1}\right)-\left(I_{4}\right)$, então as seguintes afirmações são verdadeiras:

(a) Para cada $j>k$, temos $0<\beta \leq c_{j} \leq c_{j+1}$;

(b) se $j>k$ e $c_{j}<\mathcal{S}$, então $c_{j}$ é valor crítico de $I$;

(c) se $j>k$ e $c_{j}=c_{j+1}=c_{j+2}=\ldots=c_{j+l}=c<\mathcal{S}$, então $\gamma\left(K_{c}\right) \geq l+1$.

Demonstração. Iniciamos a demonstração do teorema fazendo a seguinte afirmação que será provada posteriormente.

Afirmação 3.2 Se $j>k$ e $B \in \Gamma_{j}$, temos

$$
B \cap \partial B_{\rho} \cap X \neq \emptyset
$$

Prova de $(a)$. Uma vez que $\Gamma_{j+1} \subset \Gamma_{j}$, segue que $c_{j} \leq c_{j+1}$. Além disso, para $j>k$ e $B \in \Gamma_{j}$ temos

$$
\max _{u \in B} I(u) \geq \max _{B \cap \partial B_{\rho} \cap X} I(u) \geq \beta
$$

o que conclui a prova de $(a)$. 
Prova de $(b)$. Observe que o item (b) é uma consequência direta de $(c)$, pois aplicando o item $(c)$ com $l=0$, temos que $\gamma\left(K_{c_{j}}\right) \geq 1$, ou seja, $K_{c_{j}}$ é não vazio.

Prova de $(c)$. Para demonstrar $(c)$ vamos supor por contradição que ocorre a seguinte situação

$$
c_{j}=c_{j+1}=c_{j+2}=\ldots=c_{j+l}=c<\mathcal{S}, j>k,
$$

com $\gamma\left(K_{c}\right) \leq l$. Segue de $\left(I_{1}\right)$ e $\left(I_{3}\right)$ que $K_{c} \in \Sigma$ e $K_{c}$ é compacto. Por propriedades de gênero (ver Apêndice), existe $\delta>0$ tal que a $\delta$-vizinhança fechada, $N_{\delta}\left(K_{c}\right)$, satisfaz

$$
\gamma\left(N_{\delta}\left(K_{c}\right)\right)=\gamma\left(K_{c}\right) \leq l
$$

Por simplicidade, denotamos $N:=N_{\delta}\left(K_{c}\right)$. Pelo Lema de Deformação (ver Apêndice), existem $\eta \in C([0,1] \times E, E)$ e $\epsilon>0$ tal que $\eta(1, \cdot)$ é ímpar e vale

$$
\eta\left(1, A_{c+\epsilon} \backslash N\right) \subset A_{c-\epsilon}
$$

Escolha $B \in \Gamma_{j+l}$ de modo que

$$
\max _{u \in B} I(u) \leq c+\epsilon .
$$

Observe que por $(i i)$ temos que $\overline{B \backslash N} \in \Gamma_{j}$. Além disso, a definição de $R_{m}$ garante que $I(u) \leq 0$, para toda $u \in \partial B_{R_{m}} \cap E_{m}, \forall m \in \mathbb{N}$. Logo,

$$
u \notin I^{-1}(c-\epsilon, c+\epsilon), \quad \forall u \in \partial B_{R_{m}} \cap E_{m} .
$$

Novamente, pelo Lema de Deformação temos

$$
\eta_{1}(u):=\eta(1, u)=u, \quad \forall u \in \partial B_{R_{m}} \cap E_{m}, \quad \forall m \in \mathbb{N} .
$$

Seja $h \in G_{m}$ tal que $h\left(D_{m} \backslash Y\right)=\overline{B \backslash N} \in \Gamma_{j}$. Note que

$$
\eta_{1} \circ h \in G_{m}
$$

$\mathrm{e}$

$$
\eta(1, \overline{B \backslash N})=\eta_{1} \circ h\left(D_{m} \backslash Y\right) \in \Gamma_{j} .
$$

Por (3.9), segue que $B \subset A_{c+\epsilon}$ e, assim, $\overline{B \backslash N} \subset A_{c+\epsilon} \backslash N$. Logo, de 3.8 segue que

$$
\eta_{1}(\overline{B \backslash N}) \subset A_{c-\epsilon} .
$$

Agora pela definição de $c_{j}$ temos

$$
c=c_{j}=\inf _{B \in \Gamma_{j}} \sup _{u \in B} I(u) \leq \sup _{u \in \eta_{1}(\overline{B \backslash N})} I(u) \leq c-\epsilon<c,
$$


o que é uma contradição.

Vamos agora demonstrar a Afirmação 3.2. Considere $B=h\left(\overline{D_{m} \backslash Y}\right) \in \Gamma_{j}, j>k, m \geq$ $j$ e $\gamma(Y) \leq m-j$. Devemos mostrar que

$$
B \cap \partial B_{\rho} \cap X \neq \emptyset .
$$

Defina

$$
\Theta:=\left\{x \in D_{m} ; h(x) \in B_{\rho}\right\} .
$$

Note que $0 \in \Theta$, pois $h$ é ímpar. Vamos denotar por $\Theta_{0}$, a componente conexa de $\Theta$ que contém a origem. Uma vez que $\Theta_{0}$ é uma vizinhança limitada simétrica da origem em $E_{m}$ temos que $\gamma\left(\partial \Theta_{0}\right)=m$. Vamos assumir por um momento que

$$
h\left(\partial \Theta_{0}\right) \subset \partial B_{\rho}
$$

Considere

$$
W:=\left\{x \in D_{m} ; h(x) \in \partial B_{\rho}\right\} .
$$

Segue de 3.10$)$ que $\partial \Theta_{0} \subset W$. Daí,

$$
\gamma(W) \geq \gamma\left(\partial \Theta_{0}\right)=m .
$$

Logo,

$$
\gamma(\overline{W \backslash Y}) \geq \gamma(W)-\gamma(Y) \geq m-m+j=j>k .
$$

Por propriedades de gênero obtemos $\gamma(h(\overline{W \backslash Y}))>k$ e sendo codim $X=k$, podemos concluir que $h(\overline{W \backslash Y}) \cap X \neq \emptyset$. Mas,

$$
h(\overline{W \backslash Y}) \subset B \cap \partial B_{\rho},
$$

consequentemente,

$$
B \cap \partial B_{\rho} \cap X \neq \emptyset .
$$

Resta agora provar a afirmação $(3.10)$. Inicialmente, observe que por hipótese

$$
I(u) \leq 0, \quad \forall u \in E_{m} \backslash B_{R_{m}} .
$$

Sendo $m>k$, temos que $\partial B_{\rho} \cap X \cap E_{m} \neq \emptyset$. Portanto,

$$
I(u) \geq \beta>0, \quad \forall u \in \partial B_{\rho} \cap X \cap E_{m} .
$$

Logo, devemos ter $R_{m}>\rho$.

Suponha agora que $x \in \partial \Theta_{0}$ e $h(x) \in B_{\rho}$. Neste caso, $x \in \partial D_{m}$. Mas, em $\partial D_{m}$ temos que $h=i d$. Consequentemente, se $x \in \partial D_{m}$ e $h(x) \in B_{\rho}$, então

$$
\|h(x)\|=\|x\|=R_{m}<\rho,
$$

o que é uma contradição. 


\subsubsection{Multiplicidade de soluções}

Nos próximos lemas, mostraremos que o funcional energia $I_{\lambda}$ verifica as condições $\left(I_{2}\right)-\left(I_{4}\right)$, onde vamos considerar o caso em que $E=X=W_{0}^{1, N}(\Omega)$ e $V$ é o subespaço nulo de $W_{0}^{1, N}(\Omega)$.

Lema 3.1 O funcional $I_{\lambda}$ satisfaz as seguintes condições:

(i) Existem $\beta, \rho>0$ tais que

$$
I_{\lambda}(u) \geq \beta, \quad \text { para } \quad\|u\|=\rho .
$$

(ii) Se $\tilde{E} \subset W_{0}^{1, N}(\Omega)$ é um subespaço vetorial de dimensão finita. Então, existe

$$
R=R(\tilde{E})>0 \text { tal que }
$$

$$
I_{\lambda}(u) \leq 0, \quad \forall u \in \tilde{E} \backslash B_{R}(0)
$$

\section{Demonstração.}

Verificação de $(i)$. Recorde que, dados $\epsilon, \beta>0$ e $s \geq 1$, existe $C>0$ tal que, para cada $\alpha>\alpha_{0}$,

$$
|G(x, t)| \leq \frac{\epsilon}{N}|t|^{N}+C|t|^{s} e^{\beta|t|^{N-1}}, \quad \forall t \in \mathbb{R}
$$

e

$$
|F(x, t)| \leq \frac{\epsilon}{N}|t|^{N}+C|t|^{s} e^{\alpha|t|^{\frac{N}{N-1}}}, \quad \forall t \in \mathbb{R}
$$

Além disso, combinando a Desigualdade de Trudinger-Moser (Lema 2.1) com a imersão de Sobolev

$$
W_{0}^{1, N}(\Omega) \hookrightarrow L^{t}(\Omega), 1 \leq t<\infty,
$$

é possível mostrar que existem $\rho_{0}>0$ e uma constante $C>0$ tais que

$$
\int_{\Omega}|u|^{s} e^{\alpha|u|^{\frac{N}{N-1}}} d x \leq C|| u \|^{s}
$$

e

$$
\int_{\Omega}|u|^{s} e^{\beta|u|^{N-1}} d x \leq C|| u \|^{s}
$$

para $\|u\|<\rho_{0}(\operatorname{ver}(2.1))$. 
Destes fatos, podemos deduzir que

$$
I_{\lambda}(u) \geq \frac{1}{N}\|u\|_{*}^{N}-\left(\frac{\lambda \epsilon}{N}+\frac{\epsilon}{N}\right) \int_{\Omega}|u|^{N} d x-\lambda C\|u\|^{s}-C\|u\|^{s} .
$$

Usando a Afirmação 3.1 e imersão de Sobolev temos que, para $\epsilon>0$ suficientemente pequeno, existem constantes $C_{1}, C_{2}>0$ satisfazendo

$$
I_{\lambda}(u) \geq C_{1}\|u\|^{N}-C_{2}\|u\|^{s}, \quad \forall u \in W_{0}^{1, N}(\Omega),\|u\| \leq \rho_{0} .
$$

Considerando $s>N$, podemos fixar $0<\rho<\rho_{0}$ suficientemente pequeno verificando

$$
\beta:=C_{1} \rho^{N}-C_{2} \rho^{s}>0
$$

Portanto,

$$
I_{\lambda}(u) \geq \beta>0, \quad \text { para }\|u\|=\rho
$$

o que prova $(i)$.

Verificação de $(i i)$. Suponha que (ii) não ocorre. Logo, existe um subespaço vetorial de dimensão finita $\tilde{E} \subset W_{0}^{1, N}(\Omega)$ e existe uma sequência $\left(u_{n}\right) \subset \tilde{E} \backslash B_{n}(0)$ verificando

$$
I_{\lambda}\left(u_{n}\right)>0, \quad \forall n \in \mathbb{N}
$$

Pelas hipóteses $\left(\hat{H}_{s c}\right)$ e $\left(\hat{H}_{c}\right)$ segue que, dado $\epsilon>0$, existe $C_{\epsilon}>0$ tal que

$$
|G(x, s)| \leq \epsilon F(x, s)+C_{\epsilon}, \quad \forall s \in \mathbb{R}
$$

Por $\left(\hat{H}_{4}\right)$, existem constantes $C, D>0$ tais que

$$
F(x, s) \geq C|s|^{\nu}-D
$$

De 3.12 e 3.13 obtemos

$$
I_{\lambda}\left(u_{n}\right) \leq \frac{1}{N}|| u_{n}\left\|_{*}^{N}-(\lambda-\epsilon) \int_{\Omega} F\left(x, u_{n}\right) d x+C_{\epsilon}|\Omega| \leq C_{2}|| u_{n}\right\|^{N}-C_{3}\left|u_{n}\right|_{\nu}^{\nu}+C_{4}
$$

Sendo $\operatorname{dim} \tilde{E}<\infty$, temos que as normas $\|$.$\| e |\cdot|_{\nu}$ em $\tilde{E}$ são equivalentes. Assim, usando que $\nu>N$ e $\left\|u_{n}\right\| \rightarrow \infty$, conclui-se que

$$
I_{\lambda}\left(u_{n}\right) \rightarrow-\infty \text { quando } n \rightarrow+\infty
$$

Mas, isto contradiz (3.11), o que prova que (ii) ocorre. 
Lema 3.2 O funcional energia $I_{\lambda}$ satisfaz a condição $(P . S)_{c}$, para cada $0<c<\mathcal{S}$, onde

$$
\mathcal{S}:=\theta\left(\frac{1}{N}-\frac{1}{\nu}\right)\left(\frac{\alpha_{N}}{\alpha_{0}}\right)^{N-1}
$$

com $\theta$ dado na Afirmação 3.1 .

Demonstração. Seja $\left(u_{n}\right) \subset W_{0}^{1, N}(\Omega)$ uma sequência $(P . S)_{c}, 0<c<\mathcal{S}$. É fácil ver que

$$
\left(\frac{1}{N}-\frac{1}{\nu}\right)\left\|u_{n}\right\|_{*}^{N} \leq I_{\lambda}\left(u_{n}\right)-\frac{1}{\nu} I_{\lambda}^{\prime}\left(u_{n}\right) u_{n} \leq c+o_{n}(1)+o_{n}(1)\left\|u_{n}\right\|
$$

Logo, pela Afirmação 3.1

$$
\theta\left\|u_{n}\right\|^{N} \leq\left\|u_{n}\right\|_{*}^{N} \leq \frac{\mathcal{S}}{\left(\frac{1}{N}-\frac{1}{\nu}\right)}+o_{n}(1)+o_{n}(1)\left\|u_{n}\right\|,
$$

e usando a definição de $\mathcal{S}$, obtemos

$$
\limsup _{n \rightarrow \infty}\left\|u_{n}\right\|^{N}<\left(\frac{\alpha_{N}}{\alpha_{0}}\right)^{N-1} .
$$

Pelo Lema 2.5, existem $\alpha>\alpha_{0}, t>1$ e $C>0$, independente de $n$, tais que

$$
\int_{\Omega} e^{t \alpha\left|u_{n}\right|^{N-1}} d x \leq C, \quad \forall n \geq n_{0}
$$

para algum $n_{0}$ suficientemente grande. A prova agora segue com as mesmas idéias apresentadas no final da subseção 2.1.1. Com efeito, considere $u \in W_{0}^{1, N}(\Omega)$ como sendo o limite fraco de uma subsequência de $\left(u_{n}\right)$. Sabemos que as seguintes convergências são válidas

$$
\left\{\begin{array}{cl}
u_{n} \rightarrow u & \text { em } W_{0}^{1, N}(\Omega), \\
u_{n}(x) \rightarrow u(x) & \text { q. t. p. em } \Omega, \\
u_{n} \rightarrow u & \text { em } L^{t}(\Omega), \text { para } t \geq 1 .
\end{array}\right.
$$

Devemos justificar que os limites abaixo ocorrem.

(i) $\int_{\Omega}\left(g\left(x, u_{n}\right) u_{n}-g\left(x, u_{n}\right) u\right) d x=o_{n}(1)$;

(ii) $\int_{\Omega}\left(f\left(x, u_{n}\right) u_{n}-f\left(x, u_{n}\right) u\right) d x=o_{n}(1)$;

(iii) $\int_{\Omega}\left(a(x)\left|u_{n}\right|^{N}-a(x)\left|u_{n}\right|^{N-2} u_{n} u\right) d x=o_{n}(1)$; 
(iv) $\nabla u_{n}(x) \rightarrow \nabla u(x)$ q.t.p. em $\Omega$;

(v) $\int_{\Omega}\left(\left|\nabla u_{n}\right|^{N-2} \nabla u_{n} \nabla v-|\nabla u|^{N-2} \nabla u \nabla v\right) d x, \quad \forall v \in W_{0}^{1, N}(\Omega)$.

Os itens $(i i),(i v)$ e $(v)$ podem ser justificados de modo semelhante ao que foi visto na prova do Lema 1.8 (ver também Lema 2.6), provaremos apenas os itens $(i)$ e $(i i i)$.

Verificação de $(i)$. Observe que pelas hipóteses $\left(\hat{H}_{0}\right)$ e $\left(\hat{H}_{s c}\right)$ sobre $g$ temos que, para cada $\alpha>0$, existe uma constante $C=C(\alpha)>0$ tal que

$$
\left|g\left(x, u_{n}\right) u_{n}-g\left(x, u_{n}\right) u\right| \leq\left|u_{n}\right|^{N-1}\left|u_{n}-u\right|+C\left|u_{n}-u\right| e^{\alpha\left|u_{n}\right|^{\frac{N}{N-1}}} .
$$

Uma vez que $\left(u_{n}\right)$ é limitada, podemos supor que $\left\|u_{n}\right\| \leq C_{0}$, para alguma constante $C_{0}>0$. Considere $\alpha>0$ tal que $2 \alpha C_{0}^{\frac{N}{N-1}}<\alpha_{N}$. Logo, usando a Desigualdade de Hölder em 3.14, ficamos com

$$
\int_{\Omega}\left|g\left(x, u_{n}\right) u_{n}-g\left(x, u_{n}\right) u\right| d x \leq\left|u_{n}\right|_{N}^{N-1}\left|u_{n}-u\right|_{N}+C\left|u_{n}-u\right|_{2} \int_{\Omega} e^{2 \alpha C_{0}^{\frac{N}{N-1}}\left(\frac{\left|u_{n}\right|}{|| u_{n} \mid}\right)^{\frac{N}{N-1}}} d x .
$$

Aplicando a Desigualdade de Trudinger-Moser e usando que $u_{n} \rightarrow u$ em $L^{t}(\Omega)$, para $t \geq 1$, temos

$$
\int_{\Omega}\left|g\left(x, u_{n}\right) u_{n}-g\left(x, u_{n}\right) u\right| d x \leq C_{0}^{N-1}\left|u_{n}-u\right|_{N}+C\left|u_{n}-u\right|_{2}=o_{n}(1) .
$$

Verificação de (iii). Recorde que $a \in L^{\infty}(\Omega)$ e além disto, $\left(\left|u_{n}\right|^{N-1}\right)$ é limitada em $L^{\frac{N}{N-1}}(\Omega)$. Desta forma,

$$
\left.\int_{\Omega}|a(x)| u_{n}\right|^{N}-\left.a(x)\left|u_{n}\right|^{N-2} u_{n} u|d x \leq| a\right|_{\infty} \int_{\Omega}\left|u_{n}\right|^{N-1}\left|u_{n}-u\right| d x \leq C\left|u_{n}-u\right|_{N}=o_{n}(1) .
$$

Observe agora que

$$
\begin{aligned}
\left\|u_{n}\right\|^{N} & =I_{\lambda}^{\prime}\left(u_{n}\right) u_{n}+\int_{\Omega} a(x)\left|u_{n}\right|^{N} d x+\lambda \int_{\Omega} f\left(x, u_{n}\right) u_{n} d x+\int_{\Omega} g\left(x, u_{n}\right) u_{n} d x \\
& -I_{\lambda}\left(u_{n}\right) u+\int_{\Omega}\left|\nabla u_{n}\right|^{N-2} \nabla u_{n} \nabla u d x-\int_{\Omega} a(x)\left|u_{n}\right|^{N-2} u_{n} u d x \\
& -\lambda \int_{\Omega} f\left(x, u_{n}\right) u d x-\int_{\Omega} g\left(x, u_{n}\right) u d x
\end{aligned}
$$

Usando os limites $(i)-(v)$ temos

$$
\left\|u_{n}\right\|^{N}=\|u\|^{N}+o_{n}(1)
$$

ou seja, $u_{n} \rightarrow u$ em $W_{0}^{1, N}(\Omega)$. Portanto, $I_{\lambda}$ satisfaz a condição $(P . S)_{c}$. 
Para cada $m \in \mathbb{N}$, consideramos $E_{m} \subset W_{0}^{1, N}(\Omega)$ um subespaço vetorial de dimensão $m$. Observe que pelo Lema 3.1, existe $R_{m}>0$ tal que

$$
I_{\lambda}(u) \leq 0, \quad \forall u \in E_{m} \backslash B_{R_{m}}(0)
$$

De acordo com as notações apresentadas na subseção 3.1.1, iremos considerar os seguintes conjuntos

$$
\begin{gathered}
D_{m}:=B_{R_{m}}(0) \cap E_{m} \\
G_{m}:=\left\{h \in C\left(D_{m}, E\right) ; h \text { é ímpar e } h(u)=u, \quad \forall u \in \partial D_{m}\right\}
\end{gathered}
$$

$\mathrm{e}$

$$
\Gamma_{m}:=\left\{h\left(\overline{D_{j} \backslash Y}\right) ; h \in G_{j}, j \geq m, Y \in \Sigma \text { e } \gamma(Y) \leq j-m\right\} .
$$

Além disso, definimos

$$
c_{m}^{\lambda}=\inf _{K \in \Gamma_{m}} \max _{u \in K} I_{\lambda}(u)
$$

Lema 3.3 Para cada $m \in \mathbb{N}$, existe uma constante $0<M_{m}<\infty$ tal que

$$
c_{m}^{\lambda} \leq M_{m} \lambda^{\frac{N}{N-p}}
$$

onde $p>N$ é dado na condição $\left(\hat{H}_{5}\right)$.

Demonstração. Escolhendo $Y=\emptyset$ e $j=m$, podemos considerar $K:=\bar{D}_{m} \in \Gamma_{m}$, pois a aplicação identidade $i_{d} \in G_{m}$. Observe que

$$
c_{m}^{\lambda}=\inf _{K \in \Gamma_{m}} \max _{u \in K} I_{\lambda}(u) \leq \max _{u \in K} I_{\lambda}(u)
$$

ou ainda,

$$
c_{m}^{\lambda} \leq \max _{u \in K}\left\{\frac{1}{N}\|u\|_{*}^{N}-\lambda \int_{\Omega} F(x, u) d x-\int_{\Omega} G(x, u) d x\right\} .
$$

Dado $\epsilon>0$, existe $C_{\epsilon}>0$ tal que

$$
|G(x, u)| \leq C_{\epsilon}|u|^{N}+\epsilon F(x, u)
$$

Assim,

$$
c_{m}^{\lambda} \leq \max _{u \in K}\left\{\frac{1}{N}\|u\|_{*}^{N}-\lambda \int_{\Omega} F(x, u) d x+C_{\epsilon} \int_{\Omega}|u|^{N} d x+\epsilon \int_{\Omega} F(x, u) d x\right\} .
$$


Fazendo $\epsilon=\frac{\lambda}{2}$ e usando a hipótese $\left(\hat{H}_{5}\right)$, deduzimos

$$
c_{m}^{\lambda} \leq \max _{u \in K}\left\{\frac{1}{N}\|u\|_{*}^{N}+C_{\epsilon} \int_{\Omega}|u|^{N} d x-\frac{\lambda}{2 p} C_{p} \int_{\Omega}|u|^{p} d x\right\} .
$$

Usando 3.1 e a imersão de Sobolev $W_{0}^{1, N}(\Omega) \hookrightarrow L^{t}(\Omega), t \geq 1$, temos que existem constantes, $C_{1}>0$ e $C_{2}=C_{2}(m)>0$ tais que

$$
c_{m}^{\lambda} \leq \max _{u \in K}\left\{C_{1}\|u\|^{N}-\lambda C_{2} C_{p}\|u\|^{p}\right\}
$$

Considerando

$$
w(t)=C_{1} t^{N}-\lambda C_{2} C_{p} t^{p},
$$

temos que $w$ atinge um máximo em $t_{0}=\left(\frac{N C_{1}}{\lambda p C_{2} C_{p}}\right)^{\frac{1}{p-N}}$. Além disso,

$$
w\left(t_{0}\right)=M_{m} \lambda^{\frac{N}{N-p}}
$$

onde

$$
M_{m}:=C_{1}^{\frac{p}{p-N}}\left(C_{2} C_{p}\right)^{\frac{N}{N-p}}\left(\frac{N}{p}\right)^{\frac{N}{p-N}}\left(1-\left(\frac{N}{p}\right)\right) .
$$

Portanto,

$$
c_{m}^{\lambda} \leq M_{m} \lambda^{\frac{N}{N-p}}
$$

como queríamos demonstrar.

\section{Demonstração do Teorema 6}

Para cada $k \in \mathbb{N}$, considere $\lambda_{k}$ de maneira que

$$
M_{k}<\mathcal{S} \lambda_{k}^{\frac{N}{p-N}}
$$

Combinando o item (a) do Teorema 3.1, o Lema 3.3 e 3.16) deduzimos que, para $\lambda>\lambda_{k}$,

$$
0<c_{1}^{\lambda} \leq c_{2}^{\lambda} \leq \ldots \leq c_{k}^{\lambda} \leq M_{k} \lambda^{\frac{N}{N-p}}<M_{k} \lambda_{k}^{\frac{N}{N-p}}<\mathcal{S} .
$$

Segue do item (b) do Teorema 3.1, que os níveis

$$
c_{1}^{\lambda} \leq c_{2}^{\lambda} \leq \ldots \leq c_{k}^{\lambda}
$$

são valores críticos de $I_{\lambda}$, aos quais podemos associar pelo menos dois pontos críticos, pois o funcional $I_{\lambda}$ é par.

Observe que se $c_{j}^{\lambda}=c_{j+1}^{\lambda}$, para algum $j=1,2, \ldots, k$, então pelo item $(c)$ do Teorema 3.1 , segue que $K_{c_{j}^{\lambda}}$ é um conjunto infinito. Portanto, em qualquer dos casos, o problema $\left(P_{4}\right)$ possui pelo menos $k$ pares de soluções. 


\subsection{Multiplicidade de soluções não radiais: Quebra de}

\section{Simetria}

Nesta seção, consideramos o problema

$$
\left\{\begin{array}{c}
-\Delta_{N} u=\lambda f(|x|, u), \quad x \in \Omega_{r}, \\
u>0, \quad x \in \Omega_{r}, \\
u=0, \quad x \in \partial \Omega_{r},
\end{array}\right.
$$

onde $\Omega_{r}=\left\{x \in \mathbb{R}^{N} ; r<|x|<r+1\right\}$, com $N \geq 2, N \neq 3, r>0$ e $\lambda$ é um parâmetro positivo. Para este problema vamos assumir as seguintes hipóteses sobre a função $f$ :

$\left(\hat{\mathbf{H}}_{\mathbf{0}}\right) f \in C\left(\Omega_{r} \times \mathbb{R}, \mathbb{R}\right)$ satisfaz

$$
f(|x|, s)=o\left(|s|^{N-1}\right), \quad \text { quando } \quad s \rightarrow 0,
$$

uniformemente para $x \in \Omega_{r}$;

$\left(\hat{\mathbf{H}}_{\mathbf{c}}\right)$ existe $\alpha_{0}>0$ tal que

$$
\lim _{|s| \rightarrow \infty} \frac{|f(|x|, s)|}{e^{\alpha|s|^{N-1}}}=\left\{\begin{array}{lll}
0 & \text { se } & \alpha>\alpha_{0} \\
+\infty & \text { se } & \alpha<\alpha_{0}
\end{array}\right.
$$

uniformemente para $x \in \Omega_{r}$;

$\left(\hat{\mathbf{H}}_{4}\right)$ existe $\nu>N$ tal que

$$
0<\nu F(|x|, s) \leq f(|x|, s) s, \quad \forall|s|>0 \quad \text { e } \quad \forall x \in \Omega_{r},
$$

onde $F(|x|, s)=\int_{0}^{s} f(|x|, t) d t$

$\left(\hat{\mathbf{H}}_{\mathbf{5}}\right)$ existem $p>N$ e uma constante $C_{p}>0$ tais que

$$
f(|x|, s) \geq C_{p} s^{p-1}, \quad \forall s>0 \quad \text { e } \quad \forall x \in \Omega_{r} ;
$$

$\left(\hat{\mathbf{H}}_{\mathbf{8}}\right)$ existem $\sigma \geq N$ e uma constante $C>0$ tais que

$$
\frac{\partial f}{\partial s}(|x|, s) s-(N-1) f(|x|, s) \geq C s^{\sigma}, \quad \forall s \geq 0 \text { e } \forall x \in \Omega_{r} .
$$

Com a finalidade de obter soluções positivas assumimos que $f(|x|, s)=0$, para $s<0$. 


\subsubsection{Preliminares}

Considere $O(N)$ o grupo das matrizes reais ortogonais de ordem $N \times N$. Diremos que duas funções $u, v \in W_{0}^{1, N}\left(\Omega_{r}\right)$ são não rotacionalmente equivalentes se

$$
u(x) \neq v(g x), \quad \forall g \in O(N)
$$

Nosso objetivo é provar a existência de múltiplas soluções positivas, não radiais e não rotacionalmente equivalentes para $\left(P_{5}\right)$. Para encontrar tais soluções, analisamos o comportamento de níveis construídos sobre as variedades de Nehari em alguns subespaços de $W_{0}^{1, N}\left(\Omega_{r}\right)$ que são invariantes por alguns grupos de rotação.

Vamos inicialmente definir os espaços nos quais iremos trabalhar e estabelecer algumas notações.

Para cada inteiro $k \geq 1$, consideramos o subgrupo rotacional finito $O_{k}$ de $O(2)$ dado por $O_{k}:=\left\{g \in O(2): g(x)=\left(x_{1} \cos \frac{2 \pi l}{k}+x_{2} \operatorname{sen} \frac{2 \pi l}{k},-x_{1} \operatorname{sen} \frac{2 \pi l}{k}+x_{2} \cos \frac{2 \pi l}{k}\right), l=0,1, \ldots, k-1\right\}$, onde $x=\left(x_{1}, x_{2}\right) \in \mathbb{R}^{2}$. Definimos os subgrupos de $O(N)$

$$
G_{k}:=O_{k} \times O(N-2)
$$

e

$$
G_{\infty}:=O(2) \times O(N-2)
$$

Considere os espaços

$$
W_{0, G_{k}}^{1, N}\left(\Omega_{r}\right):=\left\{u \in W_{0}^{1, N}\left(\Omega_{r}\right) ; u(x)=u(g x), \forall g \in G_{k}\right\}, \quad k \geq 1,
$$

nos quais iremos considerar a seguinte norma

$$
\|u\|=\left(\int_{\Omega_{r}}|\nabla u|^{N} d x\right)^{1 / N}, \quad u \in W_{0, G_{k}}^{1, N}\left(\Omega_{r}\right), \quad 1 \leq k \leq \infty .
$$

No que segue, faremos uso frequente das imersões compactas (ver Apêndice Lema A.5 e Corolário A.1

$$
W_{0, G_{k}}^{1, N}\left(\Omega_{r}\right) \hookrightarrow L^{t}\left(\Omega_{r}\right), \quad 1 \leq t<\infty, 1 \leq k \leq \infty
$$

$\mathrm{e}$

$$
W_{G_{\infty}}^{1, N}\left(\mathbb{R}^{N}\right) \hookrightarrow L^{t}\left(\mathbb{R}^{N}\right), \quad N<t<\infty
$$


Para cada $k \geq 1$, encontraremos soluções em $W_{0, G_{k}}^{1, N}\left(\Omega_{r}\right)$. Para isto, associamos ao problema $\left(P_{5}\right)$ o funcional energia $I_{\lambda}: W_{0, G_{k}}^{1, N}\left(\Omega_{r}\right) \rightarrow \mathbb{R}$ dado por

$$
I_{\lambda}(u)=\frac{1}{N} \int_{\Omega_{r}}|\nabla u|^{N} d x-\lambda \int_{\Omega_{r}} F(|x|, u) d x .
$$

Para finalizar as notações, vamos definir os seguinte níveis

$$
J_{k, r}:=\inf _{u \in \mathcal{M}_{k, r}} I_{\lambda}(u)
$$

onde

$$
\mathcal{M}_{k, r}:=\left\{u \in W_{0, G_{k}}^{1, N}\left(\Omega_{r}\right) \backslash\{0\} ; I_{\lambda}^{\prime}(u) u=0\right\} .
$$

No lema seguinte, mostraremos uma versão da Desigualdade de Poincaré, que será útil ao nosso estudo.

\section{Lema 3.4 (Desigualdade de Poincaré)}

$$
\int_{\Omega_{r}}|u(z)|^{N} d z \leq\left(\frac{r+1}{r}\right)^{N-1} \int_{\Omega_{r}}|\nabla u(z)|^{N} d z
$$

para toda $u \in W_{0}^{1, N}\left(\Omega_{r}\right)$.

Demonstração. Observe que para $\psi \in C_{0}^{\infty}((r, r+1))$ temos

$$
\psi(t)=\int_{r}^{t} \psi^{\prime}(s) d s, \quad r \leq t \leq r+1 .
$$

Desta forma, aplicando a Desigualdade de Hölder

$$
|\psi(t)| \leq \int_{r}^{r+1}\left|\psi^{\prime}(s)\right| d s \leq\left(\int_{r}^{r+1}\left|\psi^{\prime}(s)\right|^{N} d s\right)^{\frac{1}{N}}\left(\int_{r}^{r+1} d s\right)^{\frac{N-1}{N}},
$$

ou seja,

$$
|\psi(t)|^{N} \leq \int_{r}^{r+1}\left|\psi^{\prime}(s)\right|^{N} d s
$$

ou ainda,

$$
\int_{r}^{r+1}|\psi(t)|^{N} d t \leq \int_{r}^{r+1}\left|\psi^{\prime}(s)\right|^{N} d s
$$

Portanto,

$$
\int_{r}^{r+1}|\psi(t)|^{N} d t \leq \int_{r}^{r+1}\left|\psi^{\prime}(t)\right|^{N} d t, \quad \forall \psi \in C_{0}^{\infty}((r, r+1)) .
$$


Considere $z=\left(\rho, \theta_{1}, \theta_{2}, \ldots, \theta_{N-1}\right)$ as coordenadas hiperesféricas de $z \in \Omega_{r}$, que consiste de uma coordenada radial $r<\rho<r+1$ e de $N-1$ coordenadas angulares $\theta_{1}, \ldots \theta_{N-1}$, $0 \leq \theta_{j} \leq \pi, j=1, \ldots, N-2$ e $0 \leq \theta_{N-1} \leq 2 \pi$. Se em coordenadas cartesianas $z=\left(z_{1}, \ldots, z_{N}\right)$, temos

$$
\begin{aligned}
z_{1} & =\rho \cos \theta_{1} \\
z_{2} & =\rho \operatorname{sen} \theta_{1} \cos \theta_{2} \\
z_{3} & =\rho \operatorname{sen} \theta_{1} \operatorname{sen} \theta_{2} \cos \theta_{3} \\
& \vdots \\
z_{N-1} & =\rho \operatorname{sen} \theta_{1} \operatorname{sen} \theta_{2} \cdots \operatorname{sen} \theta_{N-2} \cos \theta_{N-1} \\
z_{N} & =\rho \operatorname{sen} \theta_{1} \operatorname{sen} \theta_{2} \cdots \operatorname{sen} \theta_{N-2} \operatorname{sen} \theta_{N-1} .
\end{aligned}
$$

Para simplificar vamos denotar $\theta:=\left(\theta_{1}, \ldots \theta_{N-1}\right)$ e $d \theta:=d \theta_{1} d \theta_{2} \ldots d \theta_{N-1}$. Considere $\varphi \in C_{0}^{\infty}\left(\Omega_{r}\right) \cap W_{0}^{1, N}\left(\Omega_{r}\right), \varphi(z)=\varphi(\rho, \theta)$ e observe que

$$
\int_{\Omega_{r}}|\varphi(z)|^{N} d z=\int_{0}^{2 \pi} \int_{0}^{\pi} \ldots \int_{0}^{\pi} \int_{r}^{r+1}|\varphi(\rho, \theta)|^{N} \rho^{N-1} \operatorname{sen}^{N-2} \theta_{1} \operatorname{sen}^{N-3} \theta_{2} \ldots \operatorname{sen} \theta_{N-2} d \rho d \theta
$$

assim,

$$
\int_{\Omega_{r}}|\varphi(z)|^{N} d z \leq(r+1)^{N-1} \iint_{r}^{r+1}|\varphi(\rho, \theta)|^{N} \operatorname{sen}^{N-2} \theta_{1} \operatorname{sen}^{N-3} \theta_{2} \ldots \operatorname{sen} \theta_{N-2} d \rho d \theta .
$$

Fixado $\theta$, temos que a função $\psi(\rho):=\varphi(\rho, \theta)$ pertence a $C_{0}^{\infty}((r, r+1))$, assim, por (3.17) concluímos que

$$
\int_{r}^{r+1}|\psi(\rho)|^{N} d \rho \leq \int_{r}^{r+1}\left|\psi^{\prime}(\rho)\right|^{N} d \rho
$$

ou seja,

$$
\int_{r}^{r+1}|\varphi(\rho, \theta)|^{N} d \rho \leq \int_{r}^{r+1}\left|\varphi_{\rho}(\rho, \theta)\right|^{N} d \rho=\int_{r}^{r+1} \frac{1}{\rho^{N-1}}\left|\varphi_{\rho}(\rho, \theta)\right|^{N} \rho^{N-1} d \rho
$$

o que implica

$$
\int_{r}^{r+1}|\varphi(\rho, \theta)|^{N} d \rho \leq \frac{1}{r^{N-1}} \int_{r}^{r+1}\left|\varphi_{\rho}(\rho, \theta)\right|^{N} \rho^{N-1} d \rho .
$$

De (3.18) e (3.19), segue que

$$
\begin{aligned}
\int_{\Omega_{r}}|\varphi(z)|^{N} d z & \leq\left(\frac{r+1}{r}\right)^{N-1} \iint_{r}^{r+1}\left|\varphi_{\rho}(\rho, \theta)\right|^{N} \rho^{N-1} \operatorname{sen}^{N-2} \theta_{1} \operatorname{sen}^{N-3} \theta_{2} \ldots \operatorname{sen} \theta_{N-2} d \rho d \theta \\
& =\left(\frac{r+1}{r}\right)^{N-1} \iint_{r}^{r+1}\left(\left|\varphi_{\rho}(\rho, \theta)\right|^{2}\right)^{\frac{N}{2}} \rho^{N-1} \operatorname{sen}^{N-2} \theta_{1} \operatorname{sen}^{N-3} \theta_{2} \ldots \operatorname{sen} \theta_{N-2} d \rho d \theta .
\end{aligned}
$$


É fácil ver que $\varphi_{\rho}^{2} \leq|\nabla \varphi|^{2}$, portanto,

$\int_{\Omega_{r}}|\varphi(z)|^{N} d z \leq\left(\frac{r+1}{r}\right)^{N-1} \iint_{r}^{r+1}\left(|\nabla \varphi(\rho, \theta)|^{2}\right)^{\frac{N}{2}} \rho^{N-1} \operatorname{sen}^{N-2} \theta_{1} \operatorname{sen}^{N-3} \theta_{2} \ldots \operatorname{sen} \theta_{N-2} d \rho d \theta$.

Deste modo,

$$
\begin{aligned}
\int_{\Omega_{r}}|\varphi(z)|^{N} d z & \leq\left(\frac{r+1}{r}\right)^{N-1} \iint_{r}^{r+1}|\nabla \varphi(\rho, \theta)|^{N} \rho^{N-1} \operatorname{sen}^{N-2} \theta_{1} \operatorname{sen}^{N-3} \theta_{2} \ldots \operatorname{sen} \theta_{N-2} d \rho d \theta \\
& =\left(\frac{r+1}{r}\right)^{N-1} \int_{\Omega_{r}}|\nabla \varphi(z)|^{N} d z
\end{aligned}
$$

e o resultado segue por densidade.

\subsubsection{Propriedades dos níveis $J_{k, r}$}

Lema 3.5 Para cada $1 \leq k \leq \infty$ e $r>0$, temos $J_{k, r}>0$.

Demonstração. Fixados $k$ e $r$, podemos afirmar que existe $\delta>0$ tal que

$$
\|u\|^{N}>\delta, \quad \forall u \in \mathcal{M}_{k, r} .
$$

De fato, pois caso contrário, existe $\left(u_{n}\right) \subset \mathcal{M}_{k, r}$ com $\left\|u_{n}\right\| \rightarrow 0$ quando $n \rightarrow \infty$. Logo, existe $n_{0} \in \mathbb{N}$ tal que

$$
\left\|u_{n}\right\|^{N}<\left(\frac{\alpha_{N}}{\alpha_{0}}\right)^{N-1}, \forall n \geq n_{0}
$$

ou seja,

$$
\alpha_{0}\left\|u_{n}\right\|^{\frac{N}{N-1}}<\alpha_{N}, \quad \forall n \geq n_{0} .
$$

Escolha $\alpha>\alpha_{0}$ e $t_{1}>1$, de maneira que $t_{1} \alpha\left\|u_{n}\right\|^{\frac{N}{N-1}}<\alpha_{N}, \forall n \geq n_{0}$. Pelas hipóteses $\left(\hat{H}_{0}\right)$ e $\left(\hat{H}_{c}\right)$ temos que, para cada $\epsilon>0$ e $s>N$, existe $C_{\epsilon}=C(\epsilon, s)>0$ tal que

$$
\left\|u_{n}\right\|^{N}=I_{\lambda}^{\prime}\left(u_{n}\right) u_{n}+\lambda \int_{\Omega_{r}} f\left(|x|, u_{n}\right) u_{n} d x \leq \epsilon \lambda \int_{\Omega_{r}}\left|u_{n}\right|^{N} d x+\lambda C_{\epsilon} \int_{\Omega_{r}}\left|u_{n}\right|^{s} e^{\alpha\left|u_{n}\right|^{N-1}} d x .
$$

Usando Hölder, imersão de Sobolev e escolhendo $\epsilon$ suficientemente pequeno, deduzimos

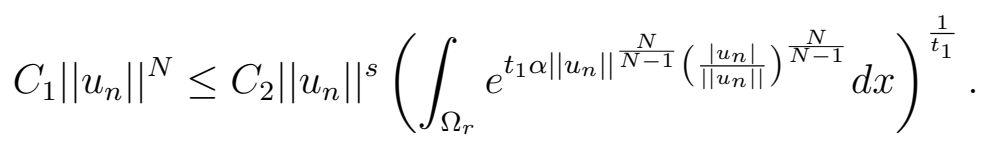

Aplicando a Desigualdade de Trudinger-Moser, temos

$$
C_{1}\left\|u_{n}\right\|^{N} \leq C_{3}\left\|u_{n}\right\|^{s}
$$


portanto, $\left\|u_{n}\right\|^{s-N} \geq C_{5}>0$, o que é uma contradição, pois $\left\|u_{n}\right\| \rightarrow 0$. Logo, $\|u\|^{N}>\delta, \quad \forall u \in \mathcal{M}_{k, r}$.

Observe que por $\left(\hat{H}_{4}\right)$, para cada $u \in \mathcal{M}_{k, r}$,

$$
I_{\lambda}(u)=I_{\lambda}(u)-\frac{1}{\nu} I_{\lambda}^{\prime}(u) u \geq\left(\frac{1}{N}-\frac{1}{\nu}\right)\|u\|^{N}>\left(\frac{1}{N}-\frac{1}{\nu}\right) \delta .
$$

Portanto,

$$
J_{k, r}>0, \quad \forall 1 \leq k \leq \infty, \forall r>0
$$

Lema 3.6 Para cada $1 \leq k<\infty$, existe $\lambda_{0}=\lambda_{0}(k)>0$, independente de $r$, tal que

$$
J_{k, r}<\frac{1}{2}\left(\frac{1}{N}-\frac{1}{\nu}\right)\left(\frac{\alpha_{N}}{\alpha_{0}}\right)^{N-1}, \forall \lambda \geq \lambda_{0} .
$$

Demonstração. Fixe $1 \leq k<\infty$. Podemos afirmar que existe $\delta=\delta(k)>0$ tal que a bola $B_{\delta, r}:=B_{\delta}\left(\left(\frac{2 r+1}{2}, 0,0, \ldots, 0\right)\right) \subset \Omega_{r}$ verifica

$$
g^{i} B_{\delta, r} \cap g^{j} B_{\delta, r}=\emptyset, \quad \forall g^{i} \in G_{k}, i \neq j, i, j=0,1,2, \ldots, k-1 .
$$

Considere $v_{r} \in W_{0}^{1, N}\left(B_{\delta, r}\right) \backslash\{0\}$ satisfazendo

$$
S_{p, k}:=\inf _{v \in W_{0}^{1, N}\left(B_{\delta, r}\right) \backslash\{0\}} \frac{\|v\|}{|v|_{p}}=\frac{\left\|v_{r}\right\|}{\left|v_{r}\right|_{p}},
$$

onde $p>N$ é dado pela hipótese $\left(\hat{H}_{5}\right)$. É importante observar que $S_{p, k}$ depende apenas de $p$ e do raio da bola $B_{\delta, r}$ que, por sua vez, depende apenas de $k$.

Defina

$$
v:=\sum_{g \in G_{k}} g v_{r} \in W_{0, G_{k}}^{1, N}\left(\Omega_{r}\right) \backslash\{0\} .
$$

Uma vez que

$$
I_{\lambda}^{\prime}(t v) t v \rightarrow-\infty \text { quando } t \rightarrow+\infty
$$

e

$$
I_{\lambda}^{\prime}(t v) t v>0, \text { para } t \approx 0
$$

existe $t_{v}>0$ tal que $t_{v} v \in \mathcal{M}_{k, r}$.

Observe que

$$
J_{k, r} \leq I_{\lambda}\left(t_{v} v\right)=k I_{\lambda}\left(t_{v} v_{r}\right)=k \max _{t \geq 0} I_{\lambda}\left(t v_{r}\right)
$$


Logo,

$$
J_{k, r} \leq k \max _{t \geq 0}\left\{\frac{t^{N}}{N}\left\|v_{r}\right\|^{N}-\lambda \int_{B_{\delta, r}} F\left(|x|, t v_{r}\right) d x\right\} .
$$

Usando a hipótese $\left(\hat{H}_{5}\right)$,

$$
J_{k, r} \leq k \max _{t \geq 0}\left\{\frac{t^{N}}{N}\left\|v_{r}\right\|^{N}-\lambda \frac{C_{p}}{p} t^{p}\left|v_{r}\right|_{p}^{p}\right\}
$$

ou seja,

$$
\frac{J_{k, r}}{\left|v_{r}\right|_{p}^{N}} \leq k \max _{t \geq 0}\left\{\frac{t^{N}}{N} S_{k, p}^{N}-\lambda \frac{C_{p}}{p} t^{p}\left|v_{r}\right|_{p}^{p-N}\right\}
$$

Considerando

$$
h(t)=\frac{t^{N}}{N} S_{k, p}^{N}-\lambda \frac{C_{p}}{p} t^{p}\left|v_{r}\right|_{p}^{p-N}
$$

temos que $h$ atinge um máximo absoluto em $t_{0}=\left[\frac{S_{k, p}^{N}}{\lambda C_{p}}\right]^{\frac{1}{p-N}} \frac{1}{\left|v_{r}\right|_{p}}$. Portanto,

$$
J_{k, r} \leq k\left(\frac{1}{N}-\frac{1}{p}\right) S_{k, p}^{\frac{N p}{p-N}} C_{p}^{\frac{N}{N-p}} \lambda^{\frac{N}{N-p}}
$$

Escolhendo

$$
\lambda_{0}=\frac{S_{k, p}^{p}}{C_{p}}\left[\frac{\frac{1}{2}\left(\frac{1}{N}-\frac{1}{\nu}\right)\left(\frac{\alpha_{N}}{\alpha_{0}}\right)^{N-1}}{k\left(\frac{1}{N}-\frac{1}{p}\right)}\right]^{\frac{N-p}{N}},
$$

temos que

$$
J_{k, r}<\frac{1}{2}\left(\frac{1}{N}-\frac{1}{\nu}\right)\left(\frac{\alpha_{N}}{\alpha_{0}}\right)^{N-1}, \forall \lambda \geq \lambda_{0},
$$

como queríamos demonstrar.

Lema 3.7 Se $\lambda \geq \lambda_{0}$ e $1 \leq k<\infty$, então $J_{k, r}$ é atingido.

Demonstração. Seja $\left(v_{n}\right) \subset \mathcal{M}_{k, r}$ uma sequência minimizante para $J_{k, r}$, isto é, $\left(v_{n}\right) \subset$ $W_{0, G_{k}}^{1, N}\left(\Omega_{r}\right) \backslash\{0\}, I_{\lambda}^{\prime}\left(v_{n}\right) v_{n}=0$ e $I_{\lambda}\left(v_{n}\right) \rightarrow J_{k, r}$. Vamos inicialmente mostrar que podemos supor

$$
I_{\lambda}^{\prime}\left(v_{n}\right) \rightarrow 0 \quad \text { em } \quad\left(W_{0, G_{k}}^{1, N}\left(\Omega_{r}\right)\right)^{\prime} .
$$

De fato, usando o Princípio Variacional de Ekeland (ver Apêndice, Teorema A.8), existe uma sequência $\left(w_{n}\right) \subset \mathcal{M}_{k, r}$ verificando

$$
w_{n}=v_{n}+o_{n}(1), \quad I_{\lambda}\left(w_{n}\right) \rightarrow J_{k, r}
$$


e

$$
I_{\lambda}^{\prime}\left(w_{n}\right)-\ell_{n} E_{\lambda}^{\prime}\left(w_{n}\right)=o_{n}(1)
$$

onde $\left(\ell_{n}\right) \subset \mathbb{R}$ e $E_{\lambda}(w)=I_{\lambda}^{\prime}(w) w$, para $w \in W_{0, G_{k}}^{1, N}\left(\Omega_{r}\right)$.

Observe que

$$
\begin{aligned}
E_{\lambda}^{\prime}\left(w_{n}\right) w_{n} & =N\left\|w_{n}\right\|^{N}-\lambda \int_{\Omega_{r}}\left[\frac{\partial f}{\partial u}\left(|x|, w_{n}\right) w_{n}^{2}+f\left(|x|, w_{n}\right) w_{n}\right] d x \\
& =N\left[I_{\lambda}^{\prime}\left(w_{n}\right) w_{n}+\lambda \int_{\Omega_{r}} f\left(|x|, w_{n}\right) w_{n} d x\right]-\lambda \int_{\Omega_{r}} \frac{\partial f}{\partial u}\left(|x|, w_{n}\right) w_{n}^{2} d x \\
& -\lambda \int_{\Omega_{r}} f\left(|x|, w_{n}\right) w_{n} d x
\end{aligned}
$$

Logo, pela hipótese $\left(\hat{H}_{8}\right)$, existem $\sigma \geq N$ e uma constante $C>0$ verificando

$$
-E_{\lambda}^{\prime}\left(w_{n}\right) w_{n}=\lambda \int_{\Omega_{r}}\left[\frac{\partial f}{\partial u}\left(|x|, w_{n}\right) w_{n}-(N-1) f\left(|x|, w_{n}\right)\right] w_{n} d x \geq C \int_{\Omega_{r}} w_{n}^{\sigma+1} d x .
$$

Com isto, podemos provar que existe $\delta>0$ tal que $\left|E_{\lambda}^{\prime}\left(w_{n}\right) w_{n}\right| \geq \delta$, para todo $n \in \mathbb{N}$. Com efeito, suponha por contradição que

$$
E_{\lambda}^{\prime}\left(w_{n}\right) w_{n}=o_{n}(1)
$$

Neste caso, de (3.21) segue que

$$
\int_{\Omega_{r}} w_{n}^{\sigma+1} d x=o_{n}(1)
$$

por interpolação

$$
\int_{\Omega_{r}} w_{n}^{\tau} d x=o_{n}(1)
$$

para todo $\tau \geq \sigma+1$.

Como podemos facilmente checar, $\left(w_{n}\right)$ é limitada e satisfaz

$$
\limsup _{n \rightarrow \infty}\left\|w_{n}\right\|^{N}<\frac{J_{k, r}}{\left(\frac{1}{N}-\frac{1}{\nu}\right)} .
$$

Logo, pelo Lema 3.6 temos que

$$
\limsup _{n \rightarrow \infty}\left\|w_{n}\right\|^{N}<\left(\frac{\alpha_{N}}{\alpha_{0}}\right)^{N-1}, \forall \lambda \geq \lambda_{0} .
$$

Como visto no Lema 2.5, podemos mostrar que existe $\alpha>\alpha_{0}, t>1(t \approx 1)$ e $C>0$, independente de $n$, tais que

$$
\int_{\Omega_{r}} e^{t \alpha\left|w_{n}\right|^{N-1}} d x \leq C, \quad \forall n \geq n_{0}
$$


Pelas hipóteses $\left(\hat{H}_{0}\right)$ e $\left(\hat{H}_{c}\right)$, segue que dados $\epsilon>0$ e $s>N$, existe $C>0$ tal que

$$
\|\left. w_{n}\right|^{N}=I_{\lambda}^{\prime}\left(w_{n}\right) w_{n}+\lambda \int_{\Omega_{r}} f\left(|x|, w_{n}\right) w_{n} d x \leq \lambda \epsilon\left|u_{n}\right|_{N}^{N}+C \int_{\Omega_{r}}\left|w_{n}\right|^{s} e^{\alpha\left|w_{n}\right|^{\frac{N}{N-1}}} d x
$$

Usando a Desigualdade de Hölder, juntamente com (3.24) e escolhendo $\epsilon$ pequeno, temos

$$
\left\|w_{n}\right\|^{N} \leq \frac{1}{2}|| w_{n} \|_{N}^{N}+C\left|w_{n}\right|_{s t_{1}}^{s}
$$

onde $t_{1}$ é o expoente conjugado de $t$. Logo, de 3.23 podemos concluir que

$$
\left\|w_{n}\right\|^{N}=o_{n}(1)
$$

ou seja, $w_{n} \rightarrow 0$ em $W_{0}^{1, N}\left(\Omega_{r}\right)$. Mas, isto não pode ocorrer pois de 3.26 temos que

$$
\left\|w_{n}\right\|^{s-N} \geq C_{2}>0
$$

Esta contradição garante que deve existir $\delta>0$ tal que

$$
\left|E_{\lambda}^{\prime}\left(w_{n}\right) w_{n}\right| \geq \delta, \quad \forall n \in \mathbb{N}
$$

Observe agora que 3.20 implica em

$$
\ell_{n} E_{\lambda}^{\prime}\left(w_{n}\right) w_{n}=o_{n}(1)
$$

assim, $\ell_{n}=o_{n}(1)$. Usando que $\left(w_{n}\right)$ é limitada e as condições de crescimento de $f$, é possível mostrar que $E_{\lambda}^{\prime}\left(w_{n}\right)$ é limitada. Novamente, de 3.20 concluímos que

$$
I_{\lambda}^{\prime}\left(w_{n}\right) \rightarrow 0 \quad \text { em } \quad\left(W_{0, G_{k}}^{1, N}\left(\Omega_{r}\right)\right)^{\prime} .
$$

Portanto, sem perda de generalidade, podemos supor que

$$
I_{\lambda}\left(v_{n}\right) \rightarrow J_{k, r} \quad \text { e } \quad I_{\lambda}^{\prime}\left(v_{n}\right) \rightarrow 0
$$

Sendo $\left(v_{n}\right)$ é limitada, existe $v \in W_{0, G_{k}}^{1, N}\left(\Omega_{r}\right)$ tal que, a menos de subsequência, temos

$$
\left\{\begin{array}{cl}
v_{n} \rightarrow v & \text { em } W_{0, G_{k}}^{1, N}\left(\Omega_{r}\right), \\
v_{n}(x) \rightarrow v(x) & \text { q. t. p. em } \Omega_{r}, \\
v_{n} \rightarrow v & \text { em } L^{t}\left(\Omega_{r}\right), \quad \text { para } t \geq 1
\end{array}\right.
$$

Além disso, os seguintes limites são verdadeiros 
(i) $\int_{\Omega_{r}}\left(f\left(|x|, v_{n}\right) v_{n}-f\left(|x|, v_{n}\right) v\right) d x=o_{n}(1)$;

(ii) $\nabla v_{n}(x) \rightarrow \nabla v(x)$ q.t.p. em $\Omega_{r}$;

(iii) $\int_{\Omega_{r}}\left(\left|\nabla v_{n}\right|^{N-2} \nabla v_{n} \nabla w-|\nabla v|^{N-2} \nabla v \nabla w\right) d x=o_{n}(1), \quad \forall w \in W_{0, G_{k}}^{1, N}\left(\Omega_{r}\right)$.

Note que as afirmações $(i)$ - $(i i i)$ são suficientes para mostrar que

$$
\left\|v_{n}\right\|^{N}=\|v\|^{N}+o_{n}(1)
$$

isto é, $v_{n} \rightarrow v$ em $W_{0, G_{k}}^{1, N}\left(\Omega_{r}\right)($ ver 2.10$)$. Logo,

$$
I_{\lambda}\left(v_{n}\right) \rightarrow I_{\lambda}(v)=J_{k, r}>0
$$

e

$$
I_{\lambda}^{\prime}\left(v_{n}\right) \rightarrow I_{\lambda}^{\prime}(v)=0
$$

Portanto, $v \in \mathcal{M}_{k, r}$ e $I_{\lambda}(v)=J_{k, r}$.

Lema 3.8 Existe $r_{0}>0$ tal que

$$
J_{\infty, r} \geq \frac{1}{2}\left(\frac{1}{N}-\frac{1}{\nu}\right)\left(\frac{\alpha_{N}}{\alpha_{0}}\right)^{N-1}, \quad \forall r>r_{0} \quad \text { e } \forall \lambda>0 .
$$

Demonstração. Suponha por contradição que existe uma sequência $\left(r_{n}\right)$, com $r_{n} \rightarrow+\infty$ quando $n \rightarrow+\infty$, verificando

$$
J_{\infty, r_{n}}<\frac{1}{2}\left(\frac{1}{N}-\frac{1}{\nu}\right)\left(\frac{\alpha_{N}}{\alpha_{0}}\right)^{N-1}, \forall n
$$

Afirmamos que $J_{\infty, r_{n}}$ é atingido, para todo $n \in \mathbb{N}$. De fato, fixado $n$, consideramos $\left(v_{k}\right) \subset \mathcal{M}_{\infty, r_{n}}$ uma sequência minimizante para $J_{\infty, r_{n}}$, ou seja, $\left(v_{k}\right) \subset W_{0, G_{\infty}}^{1, N}\left(\Omega_{r_{n}}\right) \backslash\{0\}$ satisfaz

$$
I_{\lambda}^{\prime}\left(v_{k}\right) v_{k}=0 \quad \text { e } \quad I_{\lambda}\left(v_{k}\right) \rightarrow J_{\infty, r_{n}}, \text { quando } k \rightarrow+\infty .
$$

Observe que,

$\frac{1}{2}\left(\frac{1}{N}-\frac{1}{\nu}\right)\left(\frac{\alpha_{N}}{\alpha_{0}}\right)^{N-1}>J_{\infty, r_{n}}=o_{k}(1)+I_{\lambda}\left(v_{k}\right)-\frac{1}{\nu} I_{\lambda}^{\prime}\left(v_{k}\right) v_{k} \geq o_{k}(1)+\left(\frac{1}{N}-\frac{1}{\nu}\right)\left\|v_{k}\right\|^{N}$.

Portanto,

$$
\limsup _{k \rightarrow \infty}\left\|v_{k}\right\|^{N}<\left(\frac{\alpha_{N}}{\alpha_{0}}\right)^{N-1}
$$


Uma vez que 3.30 ocorre, podemos repetir os argumentos utilizados no Lema 3.7, onde aplicamos o Princípio Variacional de Ekeland para justificar que vale

$$
I_{\lambda}^{\prime}\left(v_{k}\right) \rightarrow 0 \mathrm{em}\left(W_{0, G_{\infty}}^{1, N}\left(\Omega_{r_{n}}\right)\right)^{\prime}
$$

Seja $v \in W_{0, G_{\infty}}^{1, N}\left(\Omega_{r_{n}}\right)$ tal que $v_{k} \rightarrow v$ em $W_{0, G_{\infty}}^{1, N}\left(\Omega_{r_{n}}\right)$. Procedendo-se de modo análogo à prova do Lema 3.7, obtemos $v_{k} \rightarrow v$ em $W_{0, G_{\infty}}^{1, N}\left(\Omega_{r_{n}}\right)$. Logo,

$$
I_{\lambda}\left(v_{k}\right) \rightarrow I_{\lambda}(v)=J_{\infty, r_{n}}>0
$$

e

$$
I_{\lambda}^{\prime}\left(v_{k}\right) \rightarrow I_{\lambda}^{\prime}(v)=0
$$

Portanto, $v \in \mathcal{M}_{\infty, r_{n}}$ e $I_{\lambda}(v)=J_{\infty, r_{n}}$, provando que $J_{\infty, r_{n}}$ é atingido.

Uma vez que $J_{\infty, r_{n}}$ é atingido, para todo $n \in \mathbb{N}$, podemos escolher uma sequência $\left(u_{n}\right) \subset W_{0, G_{\infty}}^{1, N}\left(\Omega_{r_{n}}\right) \backslash\{0\}$ satisfazendo

$$
I_{\lambda}^{\prime}\left(u_{n}\right) u_{n}=0 \quad \text { e } I_{\lambda}\left(u_{n}\right)=J_{\infty, r_{n}}
$$

Logo,

$$
\frac{1}{2}\left(\frac{1}{N}-\frac{1}{\nu}\right)\left(\frac{\alpha_{N}}{\alpha_{0}}\right)^{N-1}>J_{\infty, r_{n}}=I_{\lambda}\left(u_{n}\right)-\frac{1}{\nu} I_{\lambda}^{\prime}\left(u_{n}\right) u_{n} \geq\left(\frac{1}{N}-\frac{1}{\nu}\right)\left\|u_{n}\right\|^{N}
$$

Portanto,

$$
\limsup _{n \rightarrow \infty}\left\|u_{n}\right\|^{N}<\left(\frac{\alpha_{N}}{\alpha_{0}}\right)^{N-1}
$$

Considere a sequência dada pelas extensões

$$
\tilde{u}_{n}(x)= \begin{cases}u_{n}(x), & \text { se } x \in \Omega_{r_{n}}, \\ 0, & \text { se } x \notin \Omega_{r_{n}} .\end{cases}
$$

Neste caso, temos que $\left(\tilde{u}_{n}\right)$ verifica

- $\left(\tilde{u}_{n}\right) \subset W_{G_{\infty}}^{1, N}\left(\mathbb{R}^{N}\right)$

- $\left\|\tilde{u}_{n}\right\|_{W_{G_{\infty}}^{1, N}\left(\mathbb{R}^{N}\right)}=\left\|u_{n}\right\|_{W_{0, G_{\infty}}^{1, N}\left(\Omega_{r_{n}}\right)} ;$

- $\tilde{u}_{n} \rightarrow 0$ em $W_{G_{\infty}}^{1, N}\left(\mathbb{R}^{N}\right)$, pois $\tilde{u}_{n}(x) \rightarrow 0$ q.t.p. em $\mathbb{R}^{N}$. 
Pela imersão compacta (ver Apêndice, Corolário A.1

$$
W_{G_{\infty}}^{1, N}\left(\mathbb{R}^{N}\right) \hookrightarrow L^{t}\left(\mathbb{R}^{N}\right), \quad N<t<\infty
$$

temos que

$$
\tilde{u}_{n} \rightarrow 0 \text { em } L^{t}\left(\mathbb{R}^{N}\right), \text { para } N<t<\infty
$$

Observe agora que

$$
\left\|\tilde{u}_{n}\right\|_{W_{G_{\infty}}^{1, N}\left(\mathbb{R}^{N}\right)}^{N}=I_{\lambda}^{\prime}\left(u_{n}\right) u_{n}+\lambda \int_{\Omega_{r_{n}}} f\left(|x|, u_{n}\right) u_{n} d x=\lambda \int_{\mathbb{R}^{N}} f\left(|x|, \tilde{u}_{n}\right) \tilde{u}_{n} d x .
$$

Sabemos, pelas condições de crescimento de $f$, que dados $\epsilon>0, q>N$ e $\alpha>\alpha_{0}$, existe $C_{\epsilon}>0$ tal que

$$
\|\left.\tilde{u}_{n}\right|_{W_{G_{\infty}}^{1, N}\left(\mathbb{R}^{N}\right)} ^{N} \leq \epsilon \lambda \int_{\Omega_{r_{n}}}\left|u_{n}\right|^{N} d x+C_{\epsilon} \lambda \int_{\mathbb{R}^{N}}\left|\tilde{u}_{n}\right|^{q}\left[e^{\alpha\left|\tilde{u}_{n}\right|^{N-1}}-S\left(\alpha, \tilde{u}_{n}\right)\right] d x .
$$

Pela Desigualdade de Poincaré,

$$
\left\|\tilde{u}_{n}\right\|_{W_{G_{\infty}}^{1, N}\left(\mathbb{R}^{N}\right)}^{N} \leq \epsilon \lambda\left(\frac{r_{n}+1}{r_{n}}\right)^{N-1} \int_{\Omega_{r_{n}}}\left|\nabla u_{n}\right|^{N} d x+C_{\epsilon} \lambda \int_{\mathbb{R}^{N}}\left|\tilde{u}_{n}\right|^{q}\left[e^{\left.\alpha\left|\tilde{u}_{n}\right|\right|^{N-1}}-S\left(\alpha, \tilde{u}_{n}\right)\right] d x .
$$

Fixando $\epsilon$ suficientemente pequeno, existem constantes positivas $C_{1}$ e $C_{2}$ tais que

$$
C_{1} \|\left.\tilde{u}_{n}\right|_{W_{G_{\infty}}^{1, N}\left(\mathbb{R}^{N}\right)} ^{N} \leq C_{2} \lambda \int_{\mathbb{R}^{N}}\left|\tilde{u}_{n}\right|^{q}\left[e^{\alpha\left|\tilde{u}_{n}\right|^{N-1}}-S\left(\alpha, \tilde{u}_{n}\right)\right] d x .
$$

Aplicando Hölder, obtemos

$$
C_{1}|| \tilde{u}_{n} \|_{W_{G \infty}^{1, N}\left(\mathbb{R}^{N}\right)}^{N} \leq C_{2} \lambda\left|\tilde{u}_{n}\right|_{q t}^{q}\left(\int_{\mathbb{R}^{N}}\left[e^{\alpha\left\|\tilde{u}_{n}\right\|^{\frac{N}{N-1}}\left(\frac{\left|\tilde{u}_{n}\right|}{\left\|\mid \tilde{u}_{n}\right\|}\right)^{\frac{N}{N-1}}}-S\left(\alpha,\left(\frac{\left|\tilde{u}_{n}\right|}{\left\|\tilde{u}_{n}\right\|}\right)\right)\right]^{t_{1}} d x\right)^{\frac{1}{t_{1}}}
$$

Uma vez que (3.31) ocorre, a Desigualdade de Trudinger-Moser na última integral implica que

$$
C_{1}|| \tilde{u}_{n} \|_{W_{G_{\infty}}^{1, N}\left(\mathbb{R}^{N}\right)}^{N} \leq C_{3} \lambda\left|\tilde{u}_{n}\right|_{q t}^{q} .
$$

Consequentemente, por 3.32

$$
\tilde{u}_{n} \rightarrow 0 \mathrm{em} W_{G_{\infty}}^{1, N}\left(\mathbb{R}^{N}\right)
$$

Por outro lado, usando a imersão de Sobolev em (3.33), existem constantes $C_{1}, C_{2}>0$, independentes de $r$, tais que

$$
C_{1}\left\|\tilde{u}_{n}\right\|_{W_{G \infty}^{1, N}\left(\mathbb{R}^{N}\right)}^{N} \leq C_{2}\left\|\tilde{u}_{n}\right\|_{W_{G_{\infty}}^{1, N}\left(\mathbb{R}^{N}\right)}^{q}
$$

ou ainda,

$$
\left\|u_{n}\right\|_{W_{G_{\infty}}^{1, N}\left(\mathbb{R}^{N}\right)} \geq C_{4}>0
$$

o que contradiz 3.34, demonstrando o lema. 
Lema 3.9 Suponha que $J_{k m, r}$ é atingido para algum $1 \leq k<\infty$ e algum $2 \leq m<\infty$ e suponha que $J_{k m, r}<J_{\infty, r}$. Então, $J_{k, r}<J_{k m, r}$.

Demonstração. Seja $u \in \mathcal{M}_{k m, r}$ tal que $I_{\lambda}(u)=J_{k m, r}$. Considere $x=(\theta, \rho)$ as coordenadas polares de $x \in \mathbb{R}^{2}$. Então, $u=u(\theta, \rho,|y|), y \in \mathbb{R}^{N-2}$. É fácil ver que

$$
|\nabla u|^{N}=\left(\frac{1}{\rho^{2}} u_{\theta}^{2}+u_{\rho}^{2}+\left|\nabla_{y} u\right|^{2}\right)^{\frac{N}{2}}
$$

Assim,

$$
\int_{\Omega_{r}}|\nabla u|^{N} d z=\iint_{r}^{r+1} \int_{0}^{2 \pi}\left(\frac{1}{\rho^{2}} u_{\theta}^{2}+u_{\rho}^{2}+\left|\nabla_{y} u\right|^{2}\right)^{\frac{N}{2}} \rho d \theta d \rho d y .
$$

Defina

$$
v(\theta, \rho,|y|):=u\left(\frac{\theta}{m}, \rho,|y|\right) .
$$

É possível mostrar as seguintes afirmações:

(i) $v \in W_{0, G_{k}}^{1, N}\left(\Omega_{r}\right)$;

(ii) $|\nabla v|^{N}=\left(\frac{1}{\rho^{2} m^{2}} u_{\theta}^{2}+u_{\rho}^{2}+\left|\nabla_{y} u\right|^{2}\right)^{\frac{N}{2}} ;$

(iii) $\int_{\Omega_{r}} F(v)=\int_{\Omega_{r}} F(u)$.

Sabemos que existe $t_{0}>0$ tal que $t_{0} v \in \mathcal{M}_{k, r}$. Por simplicidade, denotaremos $v:=t_{0} v$. Agora, sendo $v \in \mathcal{M}_{k, r}$ temos que

$$
J_{k, r} \leq I_{\lambda}(v)=\frac{1}{N} \int_{\Omega_{r}}|\nabla v|^{N}-\lambda \int_{\Omega_{r}} F(v) .
$$

Usando (ii)-(iii), segue que

$$
J_{k, r} \leq \frac{1}{N} \iint_{r}^{r+1} \int_{0}^{2 \pi}\left(\frac{1}{m^{2} \rho^{2}} u_{\theta}^{2}+u_{\rho}^{2}+\left|\nabla_{y} u\right|^{2}\right)^{\frac{N}{2}} \rho d \theta d \rho d y-\lambda \int_{\Omega_{r}} F(u) .
$$

Sendo $I_{\lambda}(u)=J_{k m, r}<J_{\infty, r}$, temos que $u \notin W_{0, G_{\infty}}^{1, N}\left(\Omega_{r}\right)$, portanto, $u_{\theta}^{2}$ não é identicamente nula. Então, usando que $m>1$ temos

$$
\iint_{r}^{r+1} \int_{0}^{2 \pi} \frac{1}{m^{2} \rho^{2}} u_{\theta}^{2} \rho d \theta d \rho d y<\iint_{r}^{r+1} \int_{0}^{2 \pi} \frac{1}{\rho^{2}} u_{\theta}^{2} \rho d \theta d \rho d y,
$$

que juntamente com 3.36 implica em

$$
J_{k, r}<I_{\lambda}(u)=J_{k m, r}
$$

e a demonstração está completa. 


\subsubsection{Um resultado de multiplicidade}

A seguir provamos o principal resultado desta seção.

\section{Demonstração do Teorema 7}

Pelo Lema 3.6, para cada $n \in \mathbb{N}$, existe $\lambda(n)>0$ satisfazendo

$$
J_{2^{n}, r}<\frac{1}{2}\left(\frac{1}{N}-\frac{1}{\nu}\right)\left(\frac{\alpha_{N}}{\alpha_{0}}\right)^{N-1}, \forall \lambda>\lambda(n)
$$

e pelo Lema 3.8, existe $r_{0}>0$ tal que

$$
J_{\infty, r} \geq \frac{1}{2}\left(\frac{1}{N}-\frac{1}{\nu}\right)\left(\frac{\alpha_{N}}{\alpha_{0}}\right)^{N-1}, \quad \forall r>r_{0} .
$$

Desta forma,

$$
0<J_{2^{n}, r}=J_{2 \cdot 2^{n-1}, r}<\frac{1}{2}\left(\frac{1}{N}-\frac{1}{\nu}\right)\left(\frac{\alpha_{N}}{\alpha_{0}}\right)^{N-1} \leq J_{\infty, r}
$$

$\forall \lambda>\lambda(n)$ e $\forall r>r_{0}$.

Uma vez que $J_{2^{n}, r}$ é atingido, podemos aplicar o Lema 3.9 para obter

$$
J_{2^{n-1}, r}<J_{2^{n}, r}, \quad \forall \lambda>\lambda(n) \text { e } \forall r>r_{0} .
$$

Novamente, usando que $J_{2^{n-2} 2, r}$ é atingido e satisfaz

$$
J_{2^{n-2} 2, r}=J_{2^{n-1}, r}<J_{2^{n}, r}<J_{\infty, r},
$$

segue do Lema 3.9 que

$$
J_{2^{n-2}, r}<J_{2^{n-1}, r}
$$

De forma indutiva, concluímos que

$$
0<J_{2, r}<J_{2^{2}, r}<\ldots<J_{2^{n}, r}<J_{\infty, r}
$$

$\forall \lambda>\lambda(n)$ e $\forall r>r_{0}$

Como vimos na demonstração do Lema 3.7, os minimizantes de $J_{k, r}$ são pontos críticos de $I_{\lambda}$ em $W_{0, G_{k}}^{1, N}\left(\Omega_{r}\right)$. Aplicando o Princípio da Criticalidade Simétrica de Palais (ver Apêndice, Teorema A.9 segue que estes minimizantes são pontos críticos de $I_{\lambda}$ em $W_{0}^{1, N}\left(\Omega_{r}\right)$, portanto, os minimizantes de $J_{2^{m}, r}, m=1,2, \ldots, n$ são soluções de $\left(P_{5}\right)$, não radiais, não rotacionalmente equivalentes e positivas (ver Observação 2.1). 


\section{CAPÍTULO 4}

\section{MULTIPLICIDADE DE SOLUÇÕES VIA CATEGORIA DE}

\section{LUSTERNIK-SCHNIRELMAN}

Neste capítulo, aplicamos resultados da teoria de Categoria de Lusternik-Schnirelman para obter múltiplas soluções para a classe de problemas elípticos

$$
\left\{\begin{array}{l}
-\Delta_{N} u+|u|^{N-2} u=g(u), \quad x \in \Omega_{\lambda}, \\
u>0, \quad x \in \Omega_{\lambda}, \\
u=0, \quad x \in \partial \Omega_{\lambda},
\end{array}\right.
$$

onde $\Omega_{\lambda}=\lambda \Omega$, com $\Omega \subset \mathbb{R}^{N}, N \geq 2$, sendo um domínio limitado com fronteira suave e $\lambda>1$.

Sem perda de generalidade, vamos supor que $0 \in \Omega$ e vamos fixar $r>0$ de modo que os conjuntos

$$
\Omega_{+}=\left\{x \in \mathbb{R}^{N} ; d(x, \bar{\Omega}) \leq r\right\}
$$

e

$$
\Omega_{-}=\{x \in \Omega ; d(x, \partial \Omega) \geq r\}
$$

sejam homotopicamente equivalentes, isto é, existem aplicações contínuas $\varphi: \Omega_{+} \rightarrow \Omega_{-}$e $\phi: \Omega_{-} \rightarrow \Omega_{+}$tais que as compostas $\varphi \circ \phi$ e $\phi \circ \varphi$ são homotópicas as aplicações identidades em $\Omega_{-}$e $\Omega_{+}$, respectivamente. 
Adimitimos que $g$ satisfaz as seguintes hipóteses:

$\left(\mathbf{H}_{\mathbf{0}}\right) g \in C^{1}(\mathbb{R}, \mathbb{R}) \mathrm{com}$

$$
g(s)=o\left(|s|^{N-1}\right), \text { quando } s \rightarrow 0
$$

$\left(\mathbf{H}_{\mathbf{c}}\right)$ existe $\alpha_{0}>0$ tal que

$$
\lim _{|s| \rightarrow \infty} \frac{|g(s)|}{e^{\alpha|s|^{\frac{N}{N-1}}}}=\left\{\begin{array}{lll}
0, & \text { se } & \alpha>\alpha_{0} \\
+\infty, & \text { se } & \alpha<\alpha_{0}
\end{array}\right.
$$

$\left(\mathbf{H}_{4}\right)$ existe $\nu>N$ tal que

$$
0<\nu G(s) \leq g(s) s, \quad \forall s>0,
$$

onde $G(s)=\int_{0}^{s} g(t) d t$

$\left(\mathbf{H}_{\mathbf{5}}\right)$ existem $p>N$ e uma constante $C_{p}>0$ tais que

$$
g(s) \geq C_{p} s^{p-1}, \quad \forall s \geq 0
$$

onde

$$
C_{p}>S_{p}^{p}\left\{\frac{p(\nu-N)}{\nu(p-N)}\left(\frac{\alpha_{N}}{\alpha_{0}}\right)^{N-1}\right\}^{\frac{N-p}{N}}
$$

com

$$
S_{p}:=\inf _{v \in W_{0}^{1, N}\left(B_{r}(0)\right) \backslash\{0\}} \frac{\|v\|_{W_{0}^{1, N}\left(B_{r}(0)\right)}}{|v|_{L^{p}\left(B_{r}(0)\right)}} ;
$$

$\left(\mathbf{H}_{\mathbf{6}}\right) \frac{g(s)}{s^{N-1}}$ é crescente em $(0,+\infty)$;

$\left(\mathbf{H}_{\mathbf{8}}\right)$ existem $\sigma \geq N$ e uma constante $C>0$ tais que

$$
g^{\prime}(s) s-(N-1) g(s) \geq C s^{\sigma}, \quad \forall s \geq 0 .
$$

Uma vez que desejamos obter soluções positivas para $\left(P_{6}\right)$, vamos supor que $g(s)=0$, para $s<0$. 


\subsection{Preliminares}

Nesta seção, estabelecemos algumas notações e apresentamos alguns fatos importantes que serão utilizados ao longo deste capítulo.

Para analisar o problema $\left(P_{6}\right)$, usaremos alguns resultados envolvendo o problema $\left(P_{6}\right)$ em $\mathbb{R}^{N}$

$$
\left\{\begin{array}{l}
-\Delta_{N} u+|u|^{N-2} u=g(u), \quad x \in \mathbb{R}^{N}, \\
u>0, \quad x \in \mathbb{R}^{N}, \\
u \in W^{1, N}\left(\mathbb{R}^{N}\right) .
\end{array}\right.
$$

O funcional energia associado a $\left(P_{\infty}\right)$ será denotado por $I_{\infty}: W^{1, N}\left(\mathbb{R}^{N}\right) \rightarrow \mathbb{R}$ e definido por

$$
I_{\infty}(u)=\frac{1}{N} \int_{\mathbb{R}^{N}}\left(|\nabla u|^{N}+|u|^{N}\right) d x-\int_{\mathbb{R}^{N}} G(u) d x
$$

e a variedade de Nehari associada ao funcional $I_{\infty}$ é dada por

$$
\mathcal{M}_{\infty}:=\left\{u \in W^{1, N}\left(\mathbb{R}^{N}\right) \backslash\{0\} ; I_{\infty}^{\prime}(u) u=0\right\} .
$$

Um fato importante é que o nível do Passo da Montanha para o problema $\left(P_{\infty}\right)$, denotado por $c_{\infty}$, satisfaz

$$
c_{\infty}=\inf _{u \in \mathcal{M}_{\infty}} I_{\infty}(u)
$$

O problema $\left(P_{\infty}\right)$ foi estudado por Alves \& Figueiredo [12], onde os autores provaram a existência de uma função $0<w \in W^{1, N}\left(\mathbb{R}^{N}\right)$ tal que $I_{\infty}^{\prime}(w)=0$ e $I_{\infty}(w)=c_{\infty}$, ou seja, $w$ é uma solução positiva com energia mínima para $\left(P_{\infty}\right)$.

Associamos ao problema $\left(P_{6}\right)$ o funcional energia $I_{\lambda}: W_{0}^{1, N}\left(\Omega_{\lambda}\right) \rightarrow \mathbb{R}$ dado por

$$
I_{\lambda}(u)=\frac{1}{N} \int_{\Omega_{\lambda}}\left(|\nabla u|^{N}+|u|^{N}\right) d x-\int_{\Omega_{\lambda}} G(u) d x .
$$

Consideramos a variedade de Nehari

$$
\mathcal{M}_{\lambda}:=\left\{u \in W_{0}^{1, N}\left(\Omega_{\lambda}\right) \backslash\{0\} ; I_{\lambda}^{\prime}(u) u=0\right\}
$$

e representamos por $c_{\lambda}$ o nível do Passo da Montanha aplicado a $I_{\lambda}$.

Finalmente, considerando $B_{\lambda r}:=B_{\lambda r}(0)$, denotamos por $I_{\lambda, B}: W_{0}^{1, N}\left(B_{\lambda r}\right) \rightarrow \mathbb{R}$ o funcional dado por

$$
I_{\lambda, B}(u)=\frac{1}{N} \int_{B_{\lambda r}}\left(|\nabla u|^{N}+|u|^{N}\right) d x-\int_{B_{\lambda r}} G(u) d x .
$$


Definimos a variedade de Nehari

$$
\mathcal{M}_{\lambda, B}:=\left\{u \in W_{0}^{1, N}\left(B_{\lambda r}\right) \backslash\{0\} ; I_{\lambda, B}^{\prime}(u) u=0\right\}
$$

e denotamos por $b_{\lambda}$ o nível minimax do Teorema do Passo da Montanha aplicado a $I_{\lambda, B}$.

Destacamos os seguintes fatos envolvendo os níveis $b_{\lambda}$ e $c_{\lambda}$ :

$$
b_{\lambda}=\inf _{u \in \mathcal{M}_{\lambda, B}} I_{\lambda, B}(u)
$$

e

$$
c_{\lambda}=\inf _{u \in \mathcal{M}_{\lambda}} I_{\lambda}(u)
$$

Estes fatos podem ser provados com argumentos encontrados em Willem [81], Capítulo 4.

\subsection{Um resultado de compacidade}

Veremos nesta seção que, restrito a variedade de Nehari $\mathcal{M}_{\lambda}$, o funcional $I_{\lambda}$ satisfaz a condição Palais-Smale, para níveis abaixo de um determinado valor. Além disso, provamos que os pontos críticos de $I_{\lambda}$ em $\mathcal{M}_{\lambda}$, são pontos críticos de $I_{\lambda}$ em $W_{0}^{1, N}\left(\Omega_{\lambda}\right)$. Iniciamos com o seguinte lema que estabelece uma estimativa para os níveis $b_{\lambda}, c_{\lambda}$ e $c_{\infty}$.

Lema 4.1 Os valores minimax $c_{\lambda}, b_{\lambda}$ satisfazem

$$
c_{\lambda}, b_{\lambda}<\left(\frac{1}{N}-\frac{1}{\nu}\right)\left(\frac{\alpha_{N}}{\alpha_{0}}\right)^{N-1} .
$$

Demonstração. Pelas definições de $b_{\lambda}$ e $c_{\lambda}$ podemos observar que $c_{\lambda} \leq b_{\lambda}$. Desta forma, é suficiente mostrar que

$$
b_{\lambda}<\left(\frac{1}{N}-\frac{1}{\nu}\right)\left(\frac{\alpha_{N}}{\alpha_{0}}\right)^{N-1} .
$$

Considere $\varphi \in W_{0}^{1, N}\left(B_{r}(0)\right) \backslash\{0\}$ satisfazendo

$$
\frac{\|\varphi\|}{|\varphi|_{p}}=S_{p}:=\inf _{v \in W_{0}^{1, N}\left(B_{r}(0)\right) \backslash\{0\}} \frac{\|v\|}{|v|_{p}} .
$$

Uma vez que $B_{r}(0) \subset B_{\lambda r}(0)$, pois $\lambda>1$, podemos considerar $\tilde{\varphi} \in W_{0}^{1, N}\left(B_{\lambda r}(0)\right)$ sendo a extensão de $\varphi$ ao conjunto $B_{\lambda r}(0)$, que se anula fora de $B_{r}(0)$. Pela definição de $b_{\lambda}$ temos

$$
b_{\lambda} \leq \max _{t \geq 0} I_{\lambda}(t \tilde{\varphi})
$$


isto é,

$$
b_{\lambda} \leq \max _{t \geq 0}\left\{\frac{t^{N}}{N}\|\tilde{\varphi}\|^{N}-\int_{B_{r}(0)} G(t \tilde{\varphi}) d x\right\} .
$$

Usando $\left(H_{5}\right)$,

$$
\frac{b_{\lambda}}{|\tilde{\varphi}|_{p}^{N}} \leq \max _{t \geq 0}\left\{\frac{t^{N}}{N} S_{p}^{N}-\frac{C_{p}}{p} t^{p}|\tilde{\varphi}|_{p}^{p-N}\right\} .
$$

Uma vez que a função

$$
h(t)=\frac{t^{N}}{N} S_{p}^{N}-\frac{C_{p}}{p} t^{p}|\tilde{\varphi}|_{p}^{p-N}
$$

atinge máximo absoluto em

$$
t_{0}=\left(C_{p}\right)^{\frac{1}{N-p}}|\tilde{\varphi}|_{p}^{-1} S_{p}^{\frac{N}{p-N}}
$$

concluímos que

$$
b_{\lambda} \leq\left(\frac{1}{N}-\frac{1}{p}\right)\left(C_{p}\right)^{\frac{N}{N-p}} S_{p}^{\frac{p N}{p-N}}
$$

Por 4.1), segue que

$$
b_{\lambda}<\left(\frac{1}{N}-\frac{1}{\nu}\right)\left(\frac{\alpha_{N}}{\alpha_{0}}\right)^{N-1} .
$$

Observação 4.1 Pelas definições de $c_{\lambda}$ e $c_{\infty}$ temos que $c_{\infty} \leq c_{\lambda}, \forall \lambda>1$. Desta forma,

$$
c_{\infty}<\left(\frac{1}{N}-\frac{1}{\nu}\right)\left(\frac{\alpha_{N}}{\alpha_{0}}\right)^{N-1} .
$$

A proposição seguinte pode ser encontrada em Alves \& Figueiredo [12], trata-se de um resultado de compacidade na variedade de Nehari $\mathcal{M}_{\infty}$.

Proposição 4.1 Seja $\left(u_{n}\right) \subset \mathcal{M}_{\infty}$ uma sequência satisfazendo

$$
I_{\infty}\left(u_{n}\right) \rightarrow c_{\infty}
$$

Então, $\left(u_{n}\right)$ possui subsequência convergente em $W^{1, N}\left(\mathbb{R}^{N}\right)$ ou existe $\left(y_{n}\right) \subset \mathbb{R}^{N}$ com $\left|y_{n}\right| \rightarrow \infty$ tal que

$$
v_{n}(x)=u_{n}\left(x+y_{n}\right) \rightarrow v \quad \text { em } \quad W^{1, N}\left(\mathbb{R}^{N}\right),
$$

onde $v \in \mathcal{M}_{\infty}$ e $I_{\infty}(v)=c_{\infty}$. 
Demonstração. Suponha que $\left(u_{n}\right) \subset \mathcal{M}_{\infty}$ satisfaz 4.3 . Aplicando o Princípio Variacional de Ekeland e seguindo as mesmas idéias utilizadas na demonstração de (3.28), podemos supor que

$$
I_{\infty}^{\prime}\left(u_{n}\right) \rightarrow 0 \quad \text { em } \quad\left(W^{1, N}\left(\mathbb{R}^{N}\right)\right)^{\prime}
$$

Observe que

$$
\left(\frac{1}{N}-\frac{1}{\nu}\right)\left\|u_{n}\right\|^{N} \leq I_{\infty}\left(u_{n}\right)-\frac{1}{\nu} I_{\infty}^{\prime}\left(u_{n}\right) u_{n}=c_{\infty}+o_{n}(1)
$$

Logo, $\left(u_{n}\right)$ é limitada em $W^{1, N}\left(\mathbb{R}^{N}\right)$, com

$$
\limsup _{n \rightarrow \infty}\left\|u_{n}\right\|^{N}<\left(\frac{\alpha_{N}}{\alpha_{0}}\right)^{N-1}
$$

e existe $u \in W^{1, N}\left(\mathbb{R}^{N}\right)$ tal que, para alguma subsequência, $u_{n} \rightarrow u$ em $W^{1, N}\left(\mathbb{R}^{N}\right)$.

Consideramos dois casos:

Caso I: $u \neq 0$.

Com argumentos explorados anteriormente (ver Lema 1.8 ou Proposição 2.3), podemos provar os seguintes fatos:

(i) $\nabla u_{n}(x) \rightarrow \nabla u(x)$ q.t.p. em $\mathbb{R}^{N}$;

(ii) $\int_{\mathbb{R}^{N}}\left|\nabla u_{n}\right|^{N-2} \nabla u_{n} \nabla v d x \rightarrow \int_{\mathbb{R}^{N}}|\nabla u|^{N-2} \nabla u \nabla v d x, \quad \forall v \in W^{1, N}\left(\mathbb{R}^{N}\right) ;$

(iii) $\int_{\mathbb{R}^{N}}\left|u_{n}\right|^{N-2} u_{n} v d x \rightarrow \int_{\mathbb{R}^{N}}|u|^{N-2} u v d x, \quad \forall v \in W^{1, N}\left(\mathbb{R}^{N}\right) ;$

(iv) $\int_{\mathbb{R}^{N}} g\left(u_{n}\right) \varphi d x \rightarrow \int_{\mathbb{R}^{N}} g(u) \varphi d x, \forall \varphi \in C_{0}^{\infty}\left(\mathbb{R}^{N}\right) ;$

Usando estas informações, prova-se facilmente que $u$ é ponto crítico de $I_{\infty}$, o que juntamente com o Lema de Fatou implica em

$$
\begin{aligned}
c_{\infty} & \leq I_{\infty}(u)=I_{\infty}(u)-\frac{1}{\nu} I_{\infty}^{\prime}(u) u \\
& =\left(\frac{1}{N}-\frac{1}{\nu}\right)\|u\|^{N}+\int_{\mathbb{R}^{N}}\left[\frac{1}{\nu} g(u) u-G(u)\right] d x \\
& \leq \liminf _{n \rightarrow \infty}\left\{\left(\frac{1}{N}-\frac{1}{\nu}\right)\left\|u_{n}\right\|^{N}+\int_{\mathbb{R}^{N}}\left[\frac{1}{\nu} g\left(u_{n}\right) u_{n}-G\left(u_{n}\right)\right] d x\right\} \\
& =\liminf _{n \rightarrow \infty}\left\{I_{\infty}\left(u_{n}\right)-\frac{1}{\nu} I_{\infty}^{\prime}\left(u_{n}\right) u_{n}\right\}=c_{\infty} .
\end{aligned}
$$

Consequentemente,

$$
\lim _{n \rightarrow \infty}\left\|u_{n}\right\|^{N}=\lim _{n \rightarrow \infty}\|u\|^{N}
$$

de onde concluímos que $u_{n} \rightarrow u$ em $W^{1, N}\left(\mathbb{R}^{N}\right)$. 
Caso II: $u=0$.

Neste caso, existem $R, \kappa>0$ e $\left(y_{n}\right) \subset \mathbb{R}^{N}$ tais que

$$
\limsup _{n \rightarrow \infty} \int_{B_{R}\left(y_{n}\right)}\left|u_{n}\right|^{N} d x \geq \kappa
$$

De fato, caso contrário

$$
\limsup _{n \rightarrow \infty} \sup _{y \in \mathbb{R}^{N}} \int_{B_{R}(y)}\left|u_{n}\right|^{N} d x=0
$$

e por Lions (ver Apêndice),

$$
u_{n} \rightarrow 0 \text { em } L^{q}\left(\mathbb{R}^{N}\right), \text { para todo } q \in(N,+\infty)
$$

Usando as condições de crescimento $\left(H_{0}\right)$ e $\left(H_{c}\right)$ segue que, para $s>N$ e $\alpha>\alpha_{0}$

$$
\left\|u_{n}\right\|^{N}=\int_{\mathbb{R}^{N}} g\left(u_{n}\right) u_{n} d x \leq \frac{1}{2}|| u_{n} \|^{N}+C \int_{\mathbb{R}^{N}}\left|u_{n}\right|^{s}\left[e^{\alpha\left|u_{n}\right|^{\frac{N}{N-1}}}-S_{N-2}\left(\alpha, u_{n}\right)\right] d x .
$$

Aplicando a Desigualdade de Hölder e a Desigualdade de Trudinger-Moser (ver (2.45), obtemos

$$
\left\|u_{n}\right\|^{N}=\int_{\mathbb{R}^{N}} g\left(u_{n}\right) u_{n} d x \leq \frac{1}{2}|| u_{n} \|^{N}+C\left|u_{n}\right|_{t s}^{s},
$$

o que implica $\left\|u_{n}\right\| \rightarrow 0$ e consequentemente,

$$
I_{\infty}\left(u_{n}\right) \rightarrow 0=c_{\infty}>0
$$

Logo, temos uma contradição e 4.6 deve ocorrer.

Outro fato que podemos observar é que $\left|y_{n}\right| \rightarrow+\infty$ quando $n \rightarrow+\infty$, pois se $\left(y_{n}\right)$ for limitada, digamos $\left|y_{n}\right| \leq K$ teremos

$$
\int_{B_{R+K}(0)}|u|^{N} d x=\limsup _{n \rightarrow \infty} \int_{B_{R+K}(0)}\left|u_{n}\right|^{N} d x \geq \limsup _{n \rightarrow \infty} \int_{B_{R}\left(y_{n}\right)}\left|u_{n}\right|^{N} d x \geq \kappa>0,
$$

o que contradiz $u=0$.

Defina $v_{n}(x)=u_{n}\left(x+y_{n}\right)$. Usando o Teorema de Mudança de Variáveis podemos mostrar que

$$
I_{\infty}\left(v_{n}\right)=I_{\infty}\left(u_{n}\right) \quad \text { e } \quad I_{\infty}^{\prime}\left(v_{n}\right)=I_{\infty}^{\prime}\left(u_{n}\right), \quad \forall n \in \mathbb{N}
$$

Ou seja,

$$
I_{\infty}\left(v_{n}\right) \rightarrow c_{\infty} \quad \text { e } \quad I_{\infty}^{\prime}\left(v_{n}\right) \rightarrow 0, \quad \text { quando } n \rightarrow+\infty
$$


Claramente, $\left(v_{n}\right)$ é limitada em $W^{1, N}\left(\mathbb{R}^{N}\right)$, assim, existe $v \in W^{1, N}\left(\mathbb{R}^{N}\right)$ tal que $v_{n} \rightarrow v$ em $W^{1, N}\left(\mathbb{R}^{N}\right)$.

Observe que

$$
\int_{B_{R}(0)}|v(z)|^{N} d z=\limsup _{n \rightarrow \infty} \int_{B_{R}(0)}\left|v_{n}(z)\right|^{N} d z=\limsup _{n \rightarrow \infty} \int_{B_{R}\left(y_{n}\right)}\left|u_{n}(x)\right|^{N} d x \geq \kappa>0,
$$

portanto, $v \neq 0$. Repetindo o mesmo raciocínio do Caso I para a sequência $\left(v_{n}\right)$, concluímos que $v_{n} \rightarrow v$ em $W^{1, N}\left(\mathbb{R}^{N}\right)$. Logo, de 4.7 segue que $v \in \mathcal{M}_{\infty}$ e $I_{\infty}(v)=c_{\infty}$, finalizando a demonstração.

Definimos a norma da derivada da restrição de $I_{\lambda}$ a $\mathcal{M}_{\lambda}$ em $v$ por

$$
\left\|I_{\lambda}^{\prime}(v)\right\|_{*}=\sup _{y \in T_{v} \mathcal{M}_{\lambda},\|y\|=1} I_{\lambda}^{\prime}(v) y
$$

Lema 4.2 O funcional $I_{\lambda}$ restrito a variedade de Nehari $\mathcal{M}_{\lambda}$ satisfaz a condição PalaisSmale no nivel c, para todo $c<\left(\frac{1}{N}-\frac{1}{\nu}\right)\left(\frac{\alpha_{N}}{\alpha_{0}}\right)^{N-1} \cdot$ Em outras palavras, se $\left(u_{n}\right) \subset \mathcal{M}_{\lambda}$ satisfaz

$$
I_{\lambda}\left(u_{n}\right) \rightarrow c \quad e \quad\left\|I_{\lambda}^{\prime}\left(u_{n}\right)\right\|_{*} \rightarrow 0
$$

então $\left(u_{n}\right)$ possui uma subsequência fortemente convergente em $W_{0}^{1, N}\left(\Omega_{\lambda}\right)$.

Demonstração. Observe que

$$
o_{n}(1)=\left\|I_{\lambda}^{\prime}\left(u_{n}\right)\right\|_{*}=\min _{\ell \in \mathbb{R}}\left\|I_{\lambda}^{\prime}\left(u_{n}\right)-\ell E_{\lambda}^{\prime}\left(u_{n}\right)\right\|
$$

onde $E_{\lambda}(u)=I_{\lambda}^{\prime}(u) u\left(\right.$ ver [81], pág. 87). Neste caso, existe uma sequência $\left(\ell_{n}\right) \subset \mathbb{R}$ tal que

$$
I_{\lambda}^{\prime}\left(u_{n}\right)-\ell_{n} E_{\lambda}^{\prime}\left(u_{n}\right)=o_{n}(1)
$$

Argumentando de modo análogo à prova de 3.28 , podemos justificar que $I_{\lambda}^{\prime}\left(u_{n}\right) \rightarrow 0$ em $\left(W_{0}^{1, N}\left(\Omega_{\lambda}\right)\right)^{\prime}$. Desta forma, $\left(u_{n}\right)$ é uma sequência $(P . S)_{c}$ para $I_{\lambda}$ e como podemos facilmente verificar, $\left(u_{n}\right)$ satisfaz

$$
\limsup _{n \rightarrow \infty}\left\|u_{n}\right\|^{N} \leq \frac{c}{\left(\frac{1}{N}-\frac{1}{\nu}\right)}<\left(\frac{\alpha_{N}}{\alpha_{0}}\right)^{N-1}
$$


Logo, $\left(u_{n}\right)$ é limitada em $W_{0}^{1, N}\left(\Omega_{\lambda}\right)$ e deve existir $u \in W_{0}^{1, N}\left(\Omega_{\lambda}\right)$ tal que, a menos de subsequência, $u_{n} \rightarrow u$.

Com idéias exploradas anteriormente (ver final da subseção 2.1.1), mostra-se que $u$ é ponto crítico de $I_{\lambda}$, de onde segue que $u \in \mathcal{M}_{\lambda}$.

Uma vez que (4.8) se verifica, podemos combinar o Lema 2.5 com um Lema de BrézisLieb para justificar que, para $s>N$ e $\alpha>\alpha_{0}\left(\alpha \approx \alpha_{0}\right)$, temos

$$
\int_{\Omega_{\lambda}}\left|u_{n}\right|^{s} e^{\alpha\left|u_{n}\right|^{N-1}} d x \rightarrow \int_{\Omega_{\lambda}}|u|^{s} e^{\alpha|u|^{\frac{N}{N-1}}} d x .
$$

Sendo

$$
\left|g\left(u_{n}\right) u_{n}\right| \leq\left|u_{n}\right|^{N}+C\left|u_{n}\right|^{s} e^{\alpha\left|u_{n}\right|^{N-1}}
$$

o Teorema da Convergência Dominada Generalizada de Lebesgue (ver Apêndice, Teorema A.2) implica que

$$
\lim _{n \rightarrow \infty} \int_{\Omega_{\lambda}} g\left(u_{n}\right) u_{n} d x=\int_{\Omega_{\lambda}} g(u) u d x
$$

Assim,

$$
\begin{aligned}
\left\|u_{n}\right\|^{N} & =I_{\lambda}^{\prime}\left(u_{n}\right) u_{n}+\int_{\Omega_{\lambda}} g\left(u_{n}\right) u_{n} d x=\int_{\Omega_{\lambda}} g(u) u d x+o_{n}(1) \\
& =I_{\lambda}^{\prime}(u) u+\|u\|^{N}+o_{n}(1)=\|u\|^{N}+o_{n}(1) .
\end{aligned}
$$

Portanto, $u_{n} \rightarrow u$ em $W_{0}^{1, N}\left(\Omega_{\lambda}\right)$.

Lema 4.3 Se $u \in \mathcal{M}_{\lambda}$ é um ponto crítico de $I_{\lambda}$ em $\mathcal{M}_{\lambda}$, então u é um ponto crítico não trivial de $I_{\lambda}$ em $W_{0}^{1, N}\left(\Omega_{\lambda}\right)$.

Demonstração. Seja $u \in \mathcal{M}_{\lambda}$ é um ponto crítico de $I_{\lambda}$ em $\mathcal{M}_{\lambda}$. Neste caso, $u \neq 0$ e existe $\ell \in \mathbb{R}$ verificando

$$
I_{\lambda}^{\prime}(u)=\ell E_{\lambda}^{\prime}(u)
$$

onde $E_{\lambda}(u)=I_{\lambda}^{\prime}(u) u$. Uma vez que $I_{\lambda}^{\prime}(u) u=0$, temos $\ell E_{\lambda}^{\prime}(u) u=0$. De maneira similar à verificação de (3.27), podemos mostrar que existe $\delta>0$ tal que

$$
E_{\lambda}^{\prime}(u) u \leq-\delta, \quad \forall u \in \mathcal{M}_{\lambda}
$$

Portanto, devemos ter $\ell=0$, consequentemente, $I_{\lambda}^{\prime}(u)=0$. 


\subsection{Comportamento dos níveis minimax}

Nesta seção, provamos algumas propriedades relacionadas aos níveis $c_{\infty}, c_{\lambda}$ e $b_{\lambda}$. Começaremos apresentando algumas definições e notações necessárias para o nosso estudo.

Para cada $x \in \mathbb{R}^{N}$ e $R>r>0$, denotamos

$$
A_{R, r, x}:=B_{R}(x) \backslash \bar{B}_{r}(x)
$$

e definimos o funcional $J_{\lambda, x}: W_{0}^{1, N}\left(A_{\lambda R, \lambda r, x}\right) \rightarrow \mathbb{R}$ por

$$
J_{\lambda, x}(u)=\frac{1}{N} \int_{A_{\lambda R, \lambda r, x}}\left(|\nabla u|^{N}+|u|^{N}\right) d x-\int_{A_{\lambda R, \lambda r, x}} G(u) d x .
$$

Simbolizamos por $\mathcal{M}_{\lambda, x}$ a variedade de Nehari associada a $J_{\lambda, x}$, isto é,

$$
\mathcal{M}_{\lambda, x}=\left\{u \in W_{0}^{1, N}\left(A_{\lambda R, \lambda r, x}\right) \backslash\{0\} ; J_{\lambda, x}^{\prime}(u) u=0\right\} .
$$

Para cada $u \in W^{1, N}\left(\mathbb{R}^{N}\right) \backslash\{0\}$ com suporte compacto, considere

$$
\beta(u):=\frac{\int_{\mathbb{R}^{N}} x|\nabla u|^{N} d x}{\int_{\mathbb{R}^{N}}|\nabla u|^{N} d x}
$$

e defina o seguinte número

$$
a(R, r, \lambda, x):=\inf \left\{J_{\lambda, x}(u) ; \beta(u)=x, u \in \mathcal{M}_{\lambda, x}\right\} .
$$

Por simplicidade, para $x=0$ usaremos as notações

$$
J_{\lambda}:=J_{\lambda, 0}, \quad A_{\lambda R, \lambda r}:=A_{\lambda R, \lambda r, 0}, \quad \hat{\mathcal{M}}_{\lambda}:=\mathcal{M}_{\lambda, 0}
$$

e

$$
a(R, r, \lambda):=a(R, r, \lambda, 0)
$$

Lema 4.4 O número $a(R, r, \lambda)$ satisfaz

$$
\liminf _{\lambda \rightarrow+\infty} a(R, r, \lambda)>c_{\infty}
$$

Demonstração. Seja $u \in \hat{\mathcal{M}}_{\lambda} \operatorname{com} \beta(u)=0$ e considere a extensão de $u$ ao espaço $W^{1, N}\left(\mathbb{R}^{N}\right)$ que se anula fora de $A_{\lambda R, \lambda r}$, ainda denotada por $u$. Claramente, $u \in \mathcal{M}_{\infty}$, implicando que

$$
c_{\infty} \leq I_{\infty}(u)=J_{\lambda}(u) .
$$


Pela definição de $a(R, r, \lambda)$ temos

$$
c_{\infty} \leq a(R, r, \lambda)
$$

portanto,

$$
\liminf _{\lambda \rightarrow+\infty} a(R, r, \lambda) \geq c_{\infty}
$$

Suponha por contradição que

$$
\liminf _{\lambda \rightarrow+\infty} a(R, r, \lambda)=c_{\infty}
$$

Neste caso, existe uma sequência $\left(\lambda_{n}\right)$ com $\lambda_{n} \rightarrow+\infty$ verificando

$$
\lim _{n \rightarrow+\infty} a\left(R, r, \lambda_{n}\right)=c_{\infty}
$$

Assim, existe uma sequência $\left(u_{n}\right) \subset \hat{\mathcal{M}}_{\lambda_{n}}$ satisfazendo $J_{\lambda}\left(u_{n}\right) \rightarrow c_{\infty} \operatorname{com} \beta\left(u_{n}\right)=0$, para todo $n \in \mathbb{N}$. Considerando novamente as extensões, podemos supor que $\left(u_{n}\right) \subset \mathcal{M}_{\infty}$, $I_{\infty}\left(u_{n}\right) \rightarrow c_{\infty}$ e $\beta\left(u_{n}\right)=0$, para todo $n \in \mathbb{N}$. Pela Proposição 4.1, existe uma sequência $\left(y_{n}\right) \subset \mathbb{R},\left|y_{n}\right| \rightarrow+\infty$ tal que

$$
u_{n}(x)=w_{n}(x)+\Psi\left(x-y_{n}\right)
$$

onde $\Psi \in W^{1, N}\left(\mathbb{R}^{N}\right)$ é uma função positiva satisfazendo

$$
I_{\infty}(\Psi)=c_{\infty} \quad \text { e } \quad I_{\infty}^{\prime}(\Psi)=0
$$

e $\left(w_{n}\right) \subset W^{1, N}\left(\mathbb{R}^{N}\right)$ é tal que $w_{n} \rightarrow 0$ quando $n \rightarrow+\infty$. Uma vez que $I_{\lambda}$ é rotacionalmente invariante, podemos assumir que $y_{n}=\left(y_{n}^{1}, 0,0, \ldots, 0\right)$ e que $y_{n}^{1}<0$.

Defina

$$
A:=\int_{\mathbb{R}^{N}}|\nabla \Psi|^{N} d x>0
$$

Sendo $\left\|w_{n}\right\| \rightarrow 0$, segue que do Teorema da Convergência Dominada de Lebesgue que

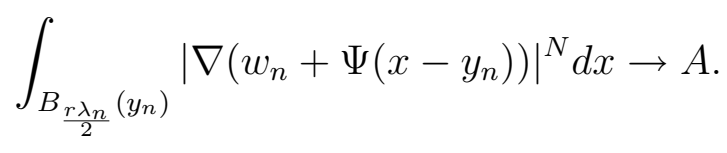

Para simplificar, usaremos as notações

$$
X_{n}:=A_{\lambda_{n} R, \lambda_{n} r} \cap B_{\frac{r \lambda_{n}}{2}}\left(y_{n}\right)
$$

e

$$
Z_{n}:=A_{\lambda_{n} R, \lambda_{n} r} \backslash B_{\frac{r \lambda_{n}}{2}}\left(y_{n}\right)
$$


Uma vez que $\left(u_{n}\right) \subset W_{0}^{1, N}\left(A_{\lambda_{n} R, \lambda_{n} r}\right)$, temos

$$
\int_{B_{\frac{r \lambda_{n}}{2}\left(y_{n}\right)}}\left|\nabla u_{n}\right|^{N} d x=\int_{A_{\lambda_{n} R, \lambda_{n} r} \cap B_{\frac{r \lambda_{n}}{2}\left(y_{n}\right)}}\left|\nabla u_{n}\right|^{N} d x=\int_{X_{n}}\left|\nabla u_{n}\right|^{N} d x,
$$

ou seja,

$$
\int_{X_{n}}\left|\nabla u_{n}\right|^{N} d x=\int_{B_{\frac{r \lambda_{n}}{2}}\left(y_{n}\right)}\left|\nabla\left(w_{n}+\Psi\left(x-y_{n}\right)\right)\right|^{N} d x \rightarrow A
$$

portanto,

$$
\int_{Z_{n}}\left|\nabla u_{n}\right|^{N} d x \rightarrow 0
$$

Sendo $\beta\left(u_{n}\right)=0$, temos

$$
0=\int_{A_{\lambda_{n} R, \lambda_{n} r}} x_{1}\left|\nabla u_{n}\right|^{N} d x=\int_{X_{n}} x_{1}\left|\nabla u_{n}\right|^{N} d x+\int_{Z_{n}} x_{1}\left|\nabla u_{n}\right|^{N} d x
$$

Da definição de $Z_{n}$ segue que

$$
\int_{Z_{n}} x_{1}\left|\nabla u_{n}\right|^{N} d x \leq R \lambda_{n} \int_{Z_{n}}\left|\nabla u_{n}\right|^{N} d x
$$

Observe que

$$
\int_{X_{n}} x_{1}\left|\nabla u_{n}\right|^{N} d x \leq \frac{-r \lambda_{n}}{2}\left(A+o_{n}(1)\right) .
$$

De fato, para $x \in X_{n}$ temos que $x \in B_{\frac{r \lambda_{n}}{2}}\left(y_{n}\right)$ e $x \notin B_{r \lambda_{n}}(0)$ que implicam, respectivamente, em

$$
\left|x_{1}-y_{n}^{1}\right|^{2}+\sum_{j=2}^{N}\left|x_{j}\right|^{2} \leq \frac{r^{2} \lambda_{n}^{2}}{4} \text { e } \sum_{j=2}^{N}\left|x_{j}\right|^{2}>r^{2} \lambda_{n}^{2}-\left|x_{1}\right|^{2} .
$$

Logo,

$$
\left|x_{1}-y_{n}^{1}\right|^{2}+r^{2} \lambda_{n}^{2}-\left|x_{1}\right|^{2} \leq \frac{r^{2} \lambda_{n}^{2}}{4}
$$

daí,

$$
\left|x_{1}\right| \geq \frac{\sqrt{3} r \lambda_{n}}{2}>\frac{r \lambda_{n}}{2}
$$

Note que não podemos ter $x_{1}>\frac{r \lambda_{n}}{2}$, portanto, deve ocorrer $x_{1}<-\frac{r \lambda_{n}}{2}$. Este fato juntamente com 4.11) implica em 4.15. Agora, usando 4.14 e 4.15 em 4.13, obtemos

$$
0=\int_{A_{\lambda_{n} R, \lambda_{n} r}} x_{1}\left|\nabla u_{n}\right|^{N} d x \leq \frac{-r \lambda_{n}}{2}(A+o(1))+R \lambda_{n} \int_{Z_{n}}\left|\nabla u_{n}\right|^{N} d x .
$$

Assim,

$$
0 \leq \frac{-r \lambda_{n}}{2}\left(A+o_{n}(1)\right)+R \lambda_{n} \int_{Z_{n}}\left|\nabla u_{n}\right|^{N} d x
$$

o que implica

$$
\int_{Z_{n}}\left|\nabla u_{n}\right|^{N} d x \geq \frac{\frac{r \lambda_{n}}{2}\left(A+o_{n}(1)\right)}{R \lambda_{n}}=\frac{r A}{2 R}+o_{n}(1),
$$

chegando a uma contradição com 4.12. 
Lema 4.5 Os números $b_{\lambda}$ e $c_{\lambda}$ verificam os seguintes limites

$$
\lim _{\lambda \rightarrow+\infty} c_{\lambda}=c_{\infty}
$$

$e$

$$
\lim _{\lambda \rightarrow+\infty} b_{\lambda}=c_{\infty}
$$

Demonstração. Considere $\xi \in C_{0}^{\infty}\left(\mathbb{R}^{N}\right), 0 \leq \xi \leq 1$, satisfazendo

$$
\xi(x)=1, x \in B_{1}(0) \quad \text { e } \quad \xi(x)=0, x \in\left(B_{2}(0)\right)^{c} .
$$

Para cada $R>0$, considere as funções

$$
\xi_{R}(x)=\xi\left(\frac{x}{R}\right) \quad \text { e } \quad \omega_{R}(x)=\xi_{R}(x) w(x),
$$

onde $w$ é uma solução com energia mínima para o problema $\left(P_{\infty}\right)$. Uma vez que $0 \in \Omega$, existe $\lambda^{*}>0$ tal que $B_{2 R}(0) \subset \Omega_{\lambda}$, para todo $\lambda \geq \lambda^{*}$.

Seja $t_{R}>0$ tal que $t_{R} \omega_{R} \in \mathcal{M}_{\lambda}$. Assim,

$$
c_{\lambda} \leq I_{\lambda}\left(t_{R} \omega_{R}\right), \quad \forall \lambda \geq \lambda^{*}
$$

e fazendo $\lambda \rightarrow+\infty$, obtemos

$$
\limsup _{\lambda \rightarrow+\infty} c_{\lambda} \leq I_{\infty}\left(t_{R} \omega_{R}\right)
$$

Afirmação $4.1 \lim _{R \rightarrow+\infty} t_{R}=1$.

Assumindo a Afirmação 4.1, temos que

$$
\lim _{R \rightarrow+\infty} I_{\infty}\left(t_{R} \omega_{R}\right)=I_{\infty}(w)=c_{\infty}
$$

assim,

$$
\limsup _{\lambda \rightarrow+\infty} c_{\lambda} \leq c_{\infty}
$$

Por outro lado, usando as definições de $c_{\lambda}$ e $c_{\infty}$ obtemos

$$
c_{\lambda} \geq c_{\infty}, \quad \forall \lambda>0
$$

o que implica

$$
\liminf _{\lambda \rightarrow+\infty} c_{\lambda} \geq c_{\infty}
$$


De 4.17) e 4.18 segue que

$$
\lim _{\lambda \rightarrow+\infty} c_{\lambda}=c_{\infty}
$$

Iremos agora provar a Afirmação 4.1. Observe que pela definição de $t_{R}$ temos $t_{R} \omega_{R} \in$ $\mathcal{M}_{\lambda}$, para todo $\lambda>\lambda^{*}$, o que implica

$$
I_{\lambda}^{\prime}\left(t_{R} \omega_{R}\right) t_{R} \omega_{R}=0
$$

ou seja,

$$
\int_{\Omega_{\lambda}}\left(\left|\nabla\left(t_{R} \omega_{R}\right)\right|^{N}+\left|t_{R} \omega_{R}\right|^{N}\right) d x=\int_{\Omega_{\lambda}} g\left(t_{R} \omega_{R}\right) t_{R} \omega_{R} d x .
$$

Observe que por $\left(H_{6}\right)$ e supondo $R>1$ temos

$$
\begin{aligned}
\int_{\mathbb{R}^{N}}\left(\left|\nabla \omega_{R}\right|^{N}+\left|\omega_{R}\right|^{N}\right) d x & =\int_{\mathbb{R}^{N}} \frac{g\left(t_{R} \omega_{R}\right)}{t_{R}^{N-1} \omega_{R}^{N-1}} \omega_{R}^{N} d x \geq \int_{B_{R}(0)} \frac{g\left(t_{R} w\right)}{t_{R}^{N-1} w^{N-1}} w^{N} d x \\
& \geq \int_{B_{1}(0)} \frac{g\left(t_{R} w\right)}{t_{R}^{N-1} w^{N-1}} w^{N} d x
\end{aligned}
$$

ou ainda,

$$
\int_{\mathbb{R}^{N}}\left(\left|\nabla \omega_{R}\right|^{N}+\left|\omega_{R}\right|^{N}\right) d x \geq \int_{B_{1}(0)} \frac{g\left(t_{R} w_{0}\right)}{t_{R}^{N-1} w_{0}^{N-1}} w_{0}^{N} d x
$$

onde $w_{0}=\min _{|x| \leq 1} w(x)$.

Note que $\left(t_{R}\right)$ é limitada, pois caso contrário, considerando $R_{n} \rightarrow+\infty \operatorname{com} t_{R_{n}} \rightarrow+\infty$, temos

$$
\int_{\mathbb{R}^{N}}\left(\left|\nabla \omega_{R_{n}}\right|^{N}+\left|\omega_{R_{n}}\right|^{N}\right) d x \geq \int_{B_{1}(0)} \frac{g\left(t_{R_{n}} w_{0}\right)}{t_{R_{n}}^{N-1} w_{0}^{N-1}} w_{0}^{N} d x .
$$

Mas, segue da hipótese $\left(H_{6}\right)$ que

$$
\frac{g\left(t_{R_{n}} w_{0}\right)}{t_{R_{n}}^{N-1} w_{0}^{N-1}} \rightarrow+\infty
$$

Assim,

$$
\lim _{n \rightarrow \infty} \int_{\mathbb{R}^{N}}\left(\left|\nabla \omega_{R_{n}}\right|^{N}+\left|\omega_{R_{n}}\right|^{N}\right) d x=+\infty
$$

Por outro lado, pelo Teorema de Lebesgue

$$
\lim _{n \rightarrow \infty} \int_{\mathbb{R}^{N}}\left(\left|\nabla \omega_{R_{n}}\right|^{N}+\left|\omega_{R_{n}}\right|^{N}\right) d x=\int_{\mathbb{R}^{N}}\left(|\nabla w|^{N}+|w|^{N}\right) d x<\infty .
$$

Esta contradição assegura que $\left(t_{R}\right)$ é limitada, deste modo,

$$
\lim _{R \rightarrow \infty} t_{R}=t_{0}<\infty .
$$


Vejamos agora que $t_{0} \neq 0$. De fato, supondo $t_{0}=0$, temos que existe $R_{n} \rightarrow \infty$ com $t_{R_{n}} \rightarrow 0$. Assim, segue das hipóteses sobre $g$ que

$$
\left|\frac{g\left(t_{R_{n}} \omega_{R_{n}}\right)}{t_{R_{n}}^{N-1} \omega_{R_{n}}^{N-1}} \omega_{R_{n}}^{N}\right| \leq \epsilon\left|\omega_{R_{n}}\right|^{N}+C_{\epsilon}\left|t_{R_{n}}\right|^{s-N+1}\left|\omega_{R_{n}}\right|^{s}\left(e^{\alpha\left|t_{R_{n}} \omega_{n}\right|^{N-1}}-S\left(\alpha, t_{R_{n}} \omega_{n}\right)\right),
$$

para $s>N$. Logo,

$$
\begin{aligned}
& \int_{\mathbb{R}^{N}}\left|\frac{g\left(t_{R_{n}} \omega_{R_{n}}\right)}{t_{R_{n}}^{N-1} \omega_{R_{n}}^{N-1}} \omega_{R_{n}}^{N}\right| \leq \epsilon\left|\omega_{R_{n}}\right|_{N}^{N}+C_{\epsilon}\left|t_{R_{n}}\right|^{s-N+1} \int_{\mathbb{R}^{N}}\left|\omega_{R_{n}}\right|^{s}\left(e^{\alpha\left|t_{R_{n}} \omega_{n}\right|^{N-1}}-S\left(\alpha, t_{R_{n}} \omega_{n}\right)\right)
\end{aligned}
$$

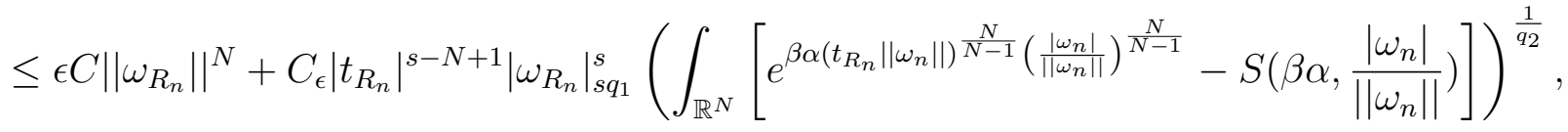

onde $q_{1}$ e $q_{2}$ são expoentes conjugados e $\beta>q_{2}$. Sendo $\omega_{R_{n}}$ limitada em $W^{1, N}\left(\mathbb{R}^{N}\right)$ e $t_{R_{n}} \rightarrow 0$, segue aplicando a Desigualdade de Trudinger-Moser que

$$
\int_{\mathbb{R}^{N}}\left|\frac{g\left(t_{R_{n}} \omega_{R_{n}}\right)}{t_{R_{n}}^{N-1} \omega_{R_{n}}^{N-1}} \omega_{R_{n}}^{N}\right| d x \leq \epsilon C+C_{\epsilon} O_{n}(1)
$$

Logo,

$$
\limsup _{n \rightarrow \infty} \int_{\mathbb{R}^{N}}\left|\frac{g\left(t_{R_{n}} \omega_{R_{n}}\right)}{t_{R_{n}}^{N-1} \omega_{R_{n}}^{N-1}} \omega_{R_{n}}^{N}\right| d x \leq \epsilon C, \quad \forall \epsilon>0
$$

isto é,

$$
\lim _{n \rightarrow \infty} \int_{\mathbb{R}^{N}} \frac{g\left(t_{R_{n}} \omega_{R_{n}}\right)}{t_{R_{n}}^{N-1} \omega_{R_{n}}^{N-1}} \omega_{R_{n}}^{N} d x=0 .
$$

Usando 4.19) e o limite anterior, concluímos que $\omega_{R_{n}} \rightarrow 0$ em $W^{1, N}\left(\mathbb{R}^{N}\right)$, o que é uma contradição com 4.23).

Do que foi visto, podemos concluir que $t_{R} \rightarrow t_{0}$ quando $R \rightarrow+\infty$, para algum $t_{0} \in(0, \infty)$ e segue de 4.19 que

$$
\int_{\mathbb{R}^{N}}\left(|\nabla w|^{N}+|w|^{N}\right) d x=\int_{\mathbb{R}^{N}} \frac{g\left(t_{0} w\right)}{t_{0}^{N-1} w^{N-1}} w^{N} d x
$$

Da última igualdade e da hipótese $\left(H_{6}\right)$ concluímos que $t_{0}=1$, provando a afirmação.

Para provar que

$$
\lim _{\lambda \rightarrow+\infty} b_{\lambda}=c_{\infty}
$$

usamos o mesmo tipo de raciocínio aplicado acima, omitiremos a demonstração. 
Lema 4.6 Existe $\hat{\lambda}>0$ tal que se $u \in \mathcal{M}_{\lambda}$ e satisfaz $I_{\lambda}(u) \leq b_{\lambda}$, então

$$
\beta(u) \in \lambda \Omega_{+}, \quad \forall \lambda \geq \hat{\lambda}
$$

Demonstração. Argumentando por contradição, suponha que existem $\lambda_{n} \rightarrow+\infty$ e $\left(u_{n}\right) \subset \mathcal{M}_{\lambda_{n}}$ com $I_{\lambda_{n}}\left(u_{n}\right) \leq b_{\lambda_{n}}$ e tal que $x_{n}=\beta\left(u_{n}\right) \notin \lambda_{n} \Omega_{+}$. Fixando $R>$ diam $\Omega$ temos

$$
\Omega_{\lambda_{n}} \subset A_{\lambda_{n} R, \lambda_{n} r, x_{n}}
$$

De fato, se $y \in \Omega_{\lambda_{n}}$ temos que $y=\lambda_{n} x \operatorname{com} x \in \Omega$. Uma vez que $x_{n} \notin \lambda_{n} \Omega_{+}$, segue que $\frac{x_{n}}{\lambda_{n}} \notin \Omega_{+}$, assim,

$$
\left|x_{n}-y\right|>r \lambda_{n}
$$

Além disso, observe que

$$
\begin{aligned}
\left|y-x_{n}\right| & =\left|\frac{\int_{\Omega_{\lambda_{n}}} y\left|\nabla u_{n}\right|^{N} d z}{\int_{\Omega_{\lambda_{n}}}\left|\nabla u_{n}\right|^{N} d z}-\frac{\int_{\Omega_{\lambda_{n}}} z\left|\nabla u_{n}\right|^{N} d z}{\int_{\Omega_{\lambda_{n}}}\left|\nabla u_{n}\right|^{N} d z}\right| \\
& =\left|\frac{\lambda_{n} \int_{\Omega_{\lambda_{n}}}\left(x-\frac{z}{\lambda_{n}}\right)\left|\nabla u_{n}\right|^{N} d z}{\int_{\Omega_{\lambda_{n}}}\left|\nabla u_{n}\right|^{N} d z}\right| \leq \lambda_{n} \operatorname{diam} \Omega \leq \lambda_{n} R .
\end{aligned}
$$

Portanto,

$$
\left|x_{n}-y\right| \leq R \lambda_{n}
$$

De 4.28 e 4.25 obtemos 4.24).

Agora, pela definição de $a\left(R, r, \lambda_{n}, x_{n}\right)$ temos

$$
a\left(R, r, \lambda_{n}, x_{n}\right) \leq b_{\lambda_{n}}
$$

e usando o fato que

$$
a\left(R, r, \lambda_{n}, x_{n}\right)=a\left(R, r, \lambda_{n}\right),
$$

concluímos que

$$
a\left(R, r, \lambda_{n}\right) \leq b_{\lambda_{n}} .
$$

Fazendo $n \rightarrow+\infty$ e aplicando o Lema 4.5 , obtemos

$$
\liminf _{n \rightarrow+\infty} a\left(R, r, \lambda_{n}\right) \leq c_{\infty},
$$

o que contradiz o Lema 4.4 . 


\subsection{Um resultado de multiplicidade}

Lema 4.7 O funcional $I_{\lambda, B}$ possui uma solução com energia mínima radialmente simétrica na origem.

Demonstração. Sabemos que o funcional $I_{\lambda, B}$ possui uma solução positiva com energia mínima, ou seja, existe $v \in W_{0}^{1, N}\left(B_{\lambda r}(0)\right)$ tal que

$$
I_{\lambda, B}(v)=b_{\lambda}=\inf _{\mathcal{M}_{\lambda}, B} I_{\lambda, B}(u) \quad \text { e } I_{\lambda, B}^{\prime}(v)=0 .
$$

Seja $v^{*}$ a simetrização de Schwartz de $v$. Neste caso, $v^{*} \in W_{0}^{1, N}\left(B_{\lambda r}(0)\right)$ e satisfaz

$$
\begin{gathered}
\int_{B_{\lambda r}(0)}\left|\nabla v^{*}\right|^{N} d x \leq \int_{B_{\lambda r}(0)}|\nabla v|^{N} d x, \\
\int_{B_{\lambda r}(0)}\left|v^{*}\right|^{N} d x=\int_{B_{\lambda r}(0)}|v|^{N} d x
\end{gathered}
$$

$\mathrm{e}$

$$
\int_{B_{\lambda r}(0)} G\left(t v^{*}\right) d x=\int_{B_{\lambda r}(0)} G(t v) d x, \quad \forall t>0 .
$$

Usando que $v \in \mathcal{M}_{\lambda, B}$, temos que $I_{\lambda, B}^{\prime}(v) v=0 \mathrm{e}$

$$
I_{\lambda, B}(v)=\max _{t \geq 0} I_{\lambda, B}(t v)
$$

Seja $t^{*}>0$ tal que $t^{*} v^{*} \in \mathcal{M}_{\lambda, B}$. Assim,

$$
b_{\lambda} \leq I_{\lambda, B}\left(t^{*} v^{*}\right) \leq I_{\lambda, B}\left(t^{*} v\right) \leq \max _{t \geq 0} I_{\lambda, B}(t v)=I_{\lambda, B}(v)=b_{\lambda}
$$

Portanto, $I_{\lambda, B}\left(t^{*} v^{*}\right)=b_{\lambda}$ e $t^{*} v^{*} \in \mathcal{M}_{\lambda B}$. Logo, $t^{*} v^{*}$ é um ponto crítico de $I_{\lambda, B}$ em $\mathcal{M}_{\lambda B}$, assim, $t^{*} v^{*}$ é um ponto crítico de $I_{\lambda, B}$ em $W_{0}^{1, N}\left(B_{\lambda r}\right)$.

Observação 4.2 No que segue, vamos denotar por $u_{\lambda, r}$ a solução positiva $t^{*} v^{*}$ de energia mínima, radialmente simétrica com relação a origem, obtida no lema anterior. Observe que $u_{\lambda, r}$ satisfaz

$$
I_{\lambda, B}\left(u_{\lambda, r}\right)=b_{\lambda}=\inf _{\mathcal{M}_{\lambda, B}} I_{\lambda, B}
$$


Para $\lambda>0$ e $r>0$, definimos o operador $\Psi_{r}: \lambda \Omega_{-} \longrightarrow W_{0}^{1, N}\left(\Omega_{\lambda}\right)$ da seguinte forma

$$
\Psi_{r}(y)(x)=\left\{\begin{array}{l}
u_{\lambda, r}(|x-y|), \quad x \in B_{\lambda r}(y), \\
0, \quad x \in \Omega_{\lambda} \backslash B_{\lambda r}(y) .
\end{array}\right.
$$

Note que,

$$
\beta\left(\Psi_{r}(y)\right)=y, \quad \forall y \in \lambda \Omega_{-} .
$$

De fato,

$$
\begin{aligned}
\beta\left(\Psi_{r}(y)\right) & =\frac{\int_{\mathbb{R}^{N}} x\left|\nabla \Psi_{r}(y)(x)\right|^{N} d x}{\int_{\mathbb{R}^{N}}\left|\nabla \Psi_{r}(y)(x)\right|^{N} d x}=\frac{\int_{B_{\lambda r}(y)} x\left|\nabla u_{\lambda, r}(|x-y|)\right|^{N} d x}{\int_{B_{\lambda r}(y)}\left|\nabla u_{\lambda, r}(|x-y|)\right|^{N} d x} \\
& =\frac{\int_{B_{\lambda r}(0)}(z+y)\left|\nabla u_{\lambda, r}(|z|)\right|^{N} d z}{\int_{B_{\lambda r}(0)}\left|\nabla u_{\lambda, r}(|z|)\right|^{N} d z}=y+\frac{\int_{B_{\lambda r}(0)} z\left|\nabla u_{\lambda, r}(|z|)\right|^{N} d z}{\int_{B_{\lambda r}(0)}\left|\nabla u_{\lambda, r}(|z|)\right|^{N} d z} .
\end{aligned}
$$

Observe que a função $H: B_{\lambda r}(0) \rightarrow \mathbb{R}$, dada por $H(z)=z_{1}\left|\nabla u_{\lambda, r}(|z|)\right|^{N}$, é uma função ímpar. Portanto,

$$
\int_{B_{\lambda r}(0)} z_{1}\left|\nabla u_{\lambda, r}(|z|)\right|^{N} d z=0
$$

o que nos conduz a 4.33 .

Lema 4.8 Para $\lambda \geq \hat{\lambda}$, temos

$$
\operatorname{cat}_{I_{\lambda}^{b_{\lambda}}}\left(I_{\lambda}^{b_{\lambda}}\right) \geq \operatorname{cat}_{\Omega_{\lambda}}\left(\Omega_{\lambda}\right)
$$

onde $I_{\lambda}^{b_{\lambda}}:=\left\{u \in W_{0}^{1, N}\left(\Omega_{\lambda}\right) ; I_{\lambda}(u) \leq b_{\lambda}\right\}$.

Demonstração. Suponha que

$$
I_{\lambda}^{b_{\lambda}}=A_{1} \cup A_{2} \cup \cdots \cup A_{n}
$$

onde $A_{j}$ é fechado e contrátil em $I_{\lambda}^{b_{\lambda}}$, para cada $j=1,2, \ldots, n$, isto é, existem $h_{j} \in$ $C\left([0,1] \times A_{j}, I_{\lambda}^{b_{\lambda}}\right)$ e $w \in A_{j}$ tais que

$$
h_{j}(0, u)=u \quad \text { e } \quad h_{j}(1, u)=w
$$

para todo $u \in A_{j}$. 
Considere $B_{j}:=\Psi_{r}^{-1}\left(A_{j}\right), 1 \leq j \leq n$. Cada conjunto $B_{j}$ é fechado, pois $\Psi_{r}$ é contínua. Além disso,

$$
\lambda \Omega_{-}=\Psi_{r}^{-1}\left(I_{\lambda}^{b_{\lambda}}\right)=B_{1} \cup B_{2} \cup \cdots \cup B_{n} .
$$

Usando a deformação $g_{j}:[0,1] \times B_{j} \rightarrow \lambda \Omega_{+}$dada por

$$
g_{i}(t, y)=\beta\left(h_{j}\left(t, \Psi_{r}(y)\right)\right)
$$

podemos concluir que $B_{j}$ é contrátil em $\lambda \Omega^{+}$. Com efeito, $g_{j}$ está bem definida, pois se $y \in B_{j}=\Psi_{r}^{-1}\left(A_{j}\right)$, temos que $\left(t, \Psi_{r}(y)\right) \in \operatorname{Dom}\left(h_{j}\right)$, para cada $(t, y) \in[0,1] \times B_{j}$. Além disso, sendo $h_{j}\left(t, \Psi_{r}(y)\right) \in I_{\lambda}^{b_{\lambda}}$ temos que

$$
h_{j}\left(t, \Psi_{r}(y)\right) \in M_{\lambda} \quad \text { e } \quad I_{\lambda}\left(h_{j}\left(t, \Psi_{r}(y)\right)\right) \leq b_{\lambda} .
$$

Pelo Lema 4.6,

$$
\beta\left(h_{j}\left(t, \Psi_{r}(y)\right)\right) \in \lambda \Omega_{+}, \quad \forall \lambda>\hat{\lambda},
$$

de onde segue que $g_{j}$ está bem definida. Note que $g_{j}$ é composição das funções contínuas $\beta$, $h_{j}$ e $\Psi, \operatorname{logo}, g_{j}$ é contínua. Observe agora que, para todo $y \in B_{j}$ temos

$$
g_{j}(0, y)=\beta\left(h_{j}\left(0, \Psi_{r}(y)\right)\right)=\beta\left(\Psi_{r}(y)\right)=y
$$

e

$$
g_{j}(0, y)=\beta\left(h_{j}\left(0, \Psi_{r}(y)\right)\right)=\beta\left(\Psi_{r}(y)\right)=y
$$

Logo, $B_{j}$ é contrátil em $\lambda \Omega^{+}, j=1,2, \ldots, n$. Portanto,

$$
\operatorname{cat}_{\Omega_{\lambda}}\left(\Omega_{\lambda}\right)=\operatorname{cat}_{\lambda \Omega_{+}}\left(\lambda \Omega_{-}\right) \leq n
$$

\section{Demonstração do Teorema 8}

Uma vez que $I_{\lambda}$ satisfaz a condição $(P . S)_{c}$ em $\mathcal{M}_{\lambda}$, podemos aplicar a teoria de LusternikSchnirelman (ver Apêndice) e o lema anterior para garantir que $I_{\lambda}$ possui pelo menos $\operatorname{cat}_{\Omega_{\lambda}}\left(\Omega_{\lambda}\right)$ de pontos críticos em $\mathcal{M}_{\lambda}$ cuja energia é menor que $b_{\lambda}$, para $\lambda \geq \lambda^{*}$. Além disso, todas as soluções obtidas são positivas (Observação 2.1). 


\section{APÊNDICE A}

\section{A.1 Desigualdades}

Desigualdades de Young. Sejam $a, b \in \mathbb{R}^{+}$e $p, p^{\prime}>1$ expoentes conjugados. Então, temos as seguintes desigualdades:

(i) $a b \leq \frac{a^{p}}{p}+\frac{b^{p^{\prime}}}{p^{\prime}}$

(ii) para cada $\epsilon>0$, temos $a b \leq \epsilon a^{p}+C_{\epsilon} b^{p^{\prime}}$, onde $C_{\epsilon}$ é uma constante positiva que depende apenas de $\epsilon, p$ e $p^{\prime}$.

ReFERÊnCIA. Evans [47], pág. 622.

Lema A.1 Sejam $x, y \in \mathbb{R}^{N}$. Então, existe uma constante $C=C(p)$ tal que

$$
\left\langle|x|^{p-2} x-|y|^{p-2} y, x-y\right\rangle \geq \begin{cases}C \frac{|x-y|^{2}}{(|x|+|y|)^{2-p}}, & \text { se } 1<p<2 \\ C|x-y|^{p}, & \text { se } p \geq 2\end{cases}
$$

onde $\langle.,$.$\rangle denota o produto interno usual do espaço \mathbb{R}^{N}$.

ReferênCia. Peral [65], pág. 78 e Simon [71]. 


\section{A.2 Resultados de convergência}

Lema A.2 (Lema de Brezis-Lieb, $1^{\circ}$ versão) Seja $\Omega$ um subconjunto aberto em $\mathbb{R}^{N} e$ seja $\left(u_{n}\right) \subset L^{p}(\Omega), 1 \leq p<\infty$. Suponha que $\left(u_{n}\right)$ é limitada em $L^{p}(\Omega)$ e $u_{n}(x) \rightarrow u(x)$ q.t.p. em $\Omega$. Então,

$$
\lim _{n \rightarrow \infty}\left(\left|u_{n}\right|_{p}^{p}-\left|u_{n}-u\right|_{p}^{p}\right)=|u|_{p}^{p}
$$

ReferÊnCIA. Kavian [53], pág. 10.

Lema A.3 (Lema de Brezis-Lieb, $2^{\circ}$ versão) Seja $\left(u_{n}\right) \subset L^{p}(\Omega), 1<p<\infty$, limitada em $L^{p}(\Omega)$ com $u_{n}(x) \rightarrow u(x)$ q.t.p. em $\Omega$. Então, $u_{n} \rightarrow u$ fracamente em $L^{p}(\Omega)$.

ReferÊnCIA. Kavian [53], pág. 11.

Teorema A.1 (Teorema da Convergência Dominada de Lebesgue) Suponha que $\left(f_{n}\right) \subset L^{1}(\Omega)$ satisfaz

(i) $f_{n}(x) \rightarrow f(x)$ q.t.p. em $\Omega$;

(ii) existe uma função $g \in L^{1}(\Omega)$ tal que, para cada $n,\left|f_{n}(x)\right| \leq g(x)$ q.t.p. em $\Omega$.

Então, $f \in L^{1}(\Omega) e\left|f_{n}-f\right|_{1} \rightarrow 0$.

REFERÊNCIA. Brezis [32], pág. 54.

Teorema A.2 (Teorema da Convergência Dominada Generalizada de Lebesgue)

Sejam $\left(f_{n}\right)$ uma sequência de funções mensuráveis e $\left(g_{n}\right) \subset L^{1}(\Omega)$ satisfazendo

(i) $f_{n}(x) \rightarrow f(x)$ q.t.p. em $\Omega$;

(ii) $g_{n}(x) \rightarrow g(x)$ q.t.p. em $\Omega$, com $g \in L^{1}(\Omega)$; 
(iii) para cada $n,\left|f_{n}(x)\right| \leq g_{n}(x)$ q.t.p. em $\Omega$;

(iv) $\left|g_{n}-g\right|_{1} \rightarrow 0$, quando $n \rightarrow \infty$.

Então, $\left|f_{n}-f\right|_{1} \rightarrow 0$.

REFERÊNCIA. Royden [68], pág. 89.

Lema A.4 (Lema de Lions) Seja $\left(u_{n}\right)$ uma sequência limitada em $W^{1, N}\left(\mathbb{R}^{N}\right)$. Suponha que existe $R>0$ tal que

$$
\lim _{n \rightarrow \infty}\left[\sup _{y \in \mathbb{R}^{N}} \int_{B_{R}(y)}\left|u_{n}(x)\right|^{N} d x\right]=0 .
$$

Então, $u_{n} \rightarrow 0$ em $L^{t}\left(\mathbb{R}^{N}\right)$, para todo $N<t<\infty$.

REFERÊNCIA. Kavian [53], pág 288.

Teorema A.3 Se E é um espaço de Banach reflexivo e $\left(u_{n}\right)$ é uma sequência limitada em $E$, então existe uma subsequência $\left(u_{n_{k}}\right)$ que converge fracamente para $u \in E$.

REFERÊNCIA. Brezis [32], pág. 50.

\section{A.3 Resultados de imersão}

Lema A.5 Seja $\Omega \subset \mathbb{R}^{N}$ um domínio limitado com fronteira regular. Então, $W^{1, N}(\Omega)$ está imerso compactamente em $L^{q}(\Omega)$, para $q \in[1,+\infty)$, isto é, o operador inclusão $i: W^{1, N}(\Omega) \rightarrow L^{q}(\Omega)$ é compacto.

REFERÊnCIA. Brezis [32], pág. 169.

Lema A.6 Seja $\Omega \subset \mathbb{R}^{N}$ um domínio regular. Então, as seguintes imersões são contínuas

$$
W^{1, N}(\Omega) \hookrightarrow L^{q}(\Omega), \quad q \in[N,+\infty)
$$


ReferênCIA. Brezis [32], pág. 168.

Definição A.1 Dados um subgrupo $G$ de $O(N), y \in \mathbb{R}^{N}$ e $r>0$, definimos o seguinte número natural:

$$
\begin{gathered}
m(y, r, G):=\sup \left\{k \in \mathbb{N} ; \text { existem } g_{1}, g_{2}, \ldots, g_{k} \in G \text { tais que } B_{r}\left(g_{i} y\right) \cap B_{r}\left(g_{j} y\right)=\emptyset\right. \\
\text { para } i \neq j, i, j=1,2, \ldots, k\} .
\end{gathered}
$$

Dizemos que $G$ é compatível com $\mathbb{R}^{N}$ se existe $r>0$ tal que

$$
\lim _{|y| \rightarrow \infty} m(y, r, G)=\infty
$$

Teorema A.4 Seja $G$ é um subgrupo de $O(N)$ compatível com $\mathbb{R}^{N}$. Então,

$$
W_{G}^{1, N}\left(\mathbb{R}^{N}\right) \underset{\operatorname{com} p}{\hookrightarrow} L^{t}\left(\mathbb{R}^{N}\right), \quad N<t<\infty
$$

onde

$$
W_{G}^{1, N}\left(\mathbb{R}^{N}\right)=\left\{u \in W^{1, N}\left(\mathbb{R}^{N}\right) ; g u=u, \forall g \in G\right\}
$$

e $g u(x)=u\left(g^{-1} x\right), x \in \mathbb{R}^{N}$

Demonstração. Seja $\left(u_{n}\right) \subset W_{G}^{1, N}\left(\mathbb{R}^{N}\right)$ tal que $u_{n} \rightarrow u$ em $W_{G}^{1, N}\left(\mathbb{R}^{N}\right)$. É suficiente mostrar que $v_{n}:=u_{n}-u \rightarrow 0$ em $L^{t}\left(\mathbb{R}^{N}\right), N<t<\infty$.

Dado $y \in \mathbb{R}^{N}$, segue da definição de $m(y, r, G)$ que, existem $g_{1}, g_{2}, \ldots, g_{m}(y, r, G) \in G$ tais que $B_{r}\left(g_{i} y\right) \cap B_{r}\left(g_{j} y\right)=\emptyset$, para $i \neq j, i, j=1,2, \ldots, m(y, r, G)$.

Sendo $\left(v_{n}\right) \subset W_{G}^{1, N}\left(\mathbb{R}^{N}\right)$, segue aplicado o Teorema de Mudança de Variáveis que

$$
\int_{B_{r}(y)}\left|v_{n}\right|^{N} d x=\int_{B_{r}\left(g_{i} y\right)}\left|v_{n}\right|^{N} d x, \quad \forall i=1,2, \ldots, m(y, r, G),
$$

assim,

$$
\begin{aligned}
\int_{B_{r}(y)}\left|v_{n}\right|^{N} d x & =\frac{1}{m(y, r, G)} \sum_{i=1}^{m(y, r, G)} \int_{B_{r}\left(g_{i} y\right)}\left|v_{n}\right|^{N} d x=\frac{1}{m(y, r, G)} \int_{\cup_{i=1}^{m(y, r, G)} B_{r}\left(g_{i} y\right)}\left|v_{n}\right|^{N} d x \\
& \leq \frac{1}{m(y, r, G)} \int_{\mathbb{R}^{N}}\left|v_{n}\right|^{N} d x \leq \frac{\sup _{n}\left\|v_{n}\right\|^{N}}{m(y, r, G)} \leq \frac{C}{m(y, r, G)}
\end{aligned}
$$


Uma vez que $G$ é compatível com $\mathbb{R}^{N}$ temos que, dado $\epsilon>0$, existe $R>0$ tal que

$$
\sup _{|y| \geq R} \int_{B_{r}(y)}\left|v_{n}\right|^{N} d x \leq \epsilon, \quad n \in \mathbb{N} .
$$

Pelo Lema A.5 temos que, para $|y| \leq R$, existe $n_{0} \in \mathbb{N}$ tal que

$$
\int_{B_{r}(y)}\left|v_{n}\right|^{N} d x \leq \int_{B_{R+r}(0)}\left|v_{n}\right|^{N} d x \leq \epsilon, \quad n \geq n_{0}
$$

Combinando $\mathrm{A} .2 \mathrm{~A}$ e $\mathrm{A} .3 \mathrm{n}$ obtemos

$$
\lim _{n \rightarrow \infty}\left[\sup _{y \in \mathbb{R}^{N}} \int_{B_{R}(y)}\left|v_{n}(x)\right|^{N} d x\right]=0 .
$$

Logo, aplicando o Lema A.4, concluímos que $v_{n} \rightarrow 0$ em $L^{t}\left(\mathbb{R}^{N}\right)$, para todo $N<t<\infty$.

Corolário A.1 Sejam $2 \leq N_{1}, N_{2}, \ldots, N_{l} \in \mathbb{N} \operatorname{com} N=N_{1}+N_{2}+\ldots+N_{l}$ e $G:=$ $O\left(N_{1}\right) \times O\left(N_{2}\right) \times \ldots \times O\left(N_{l}\right)$. Então,

$$
W_{G}^{1, N}\left(\mathbb{R}^{N}\right) \underset{\operatorname{com} p}{\hookrightarrow} L^{t}\left(\mathbb{R}^{N}\right), \quad N<t<\infty
$$

Demonstração. Basta notar que $G$ é compatível com $\mathbb{R}^{N}$ e aplicar o Teorema A.4. ReferÊnCIA. O Teorema A.4 é uma versão do Teorema 1.24 de [81] (pág.17) para o espaço $W^{1, N}\left(\mathbb{R}^{N}\right)$.

\section{A.4 Teorema de Deformação}

Teorema A.5 (Teorema de Deformação) Sejam E um espaço de Banach real e $I \in$ $C^{1}(E, \mathbb{R})$ satisfazendo a condição $(P . S)$. Se $c \in \mathbb{R}, \bar{\epsilon}>0$ e $N$ é uma vizinhança de $K_{c}$, então existe $\epsilon \in(0, \bar{\epsilon})$ e $\eta \in C([0,1] \times E, E)$ tais que

(i) $\eta(0, u)=u, \quad \forall u \in E$;

(ii) $\eta(t, u)=u$, se $I(u) \notin[c-\bar{\epsilon}, c+\bar{\epsilon}], \quad \forall t \in[0,1]$; 
(iii) $\eta(t, u)$ é um homeomorfismo de E sobre $E, \forall t \in[0,1]$;

(iv) $\eta\left(1, A_{c+\epsilon} \backslash N\right) \subset A_{c-\epsilon}$;

(v) se $K_{c}=\emptyset, \eta\left(1, A_{c+\epsilon}\right) \subset A_{c-\epsilon}$;

(vi) $\|\eta(t, u)-u\| \leq 1, u \in E, \quad \forall t \in[0,1]$

(vii) $I(\eta(t, u)) \leq I(u), u \in E, \quad \forall t \in[0,1]$;

(viii) se $I(u)$ é par em $u$, então $\eta(t, u)$ é ímpar em $u$,

onde $A_{c}:=\{u \in E ; I(u) \leq c\}$.

ReFerÊnCIA. Rabinowitz [66], pág. 82.

\section{A.5 Teorema do Passo da Montanha}

Teorema A.6 (Teorema do Passo da Montanha sem a condição Palais-Smale)

Seja $E$ um espaço de Banach e $I \in C^{1}(E, \mathbb{R})$ com $I(0)=0$. Suponha que:

(i) existe $\beta, \rho>0$ tal que

$$
I(u) \geq \beta, \quad \text { para }\|u\|=\rho ;
$$

(ii) existe $e \in E$ tal que $\|e\|>\rho$ e $I(e)<0$.

Então, existe uma sequência $\left(u_{n}\right) \in E$ tal que

$$
I\left(u_{n}\right) \rightarrow c \quad e \quad I^{\prime}\left(u_{n}\right) \rightarrow 0 \quad \text { em } \quad E^{\prime}
$$

onde

$$
0<c=\inf _{\gamma \in \Gamma} \max _{t \in[0,1]} I(\gamma(t))
$$


$e$

$$
\Gamma=\{\gamma \in C([0,1] ; E) \mid \gamma(0)=0 \text { e } \gamma(1)=e\}
$$

REFERÊNCIA. Willem [81].

\section{A.6 Princípio Variacional de Ekeland}

Teorema A.7 (Princípio Variacional de Ekeland, $1^{\circ}$ versão) Seja $(E, d)$ um espaço métrico completo com métrica d e seja $J: E \rightarrow \mathbb{R} \cup\{+\infty\}$ uma função semicontínua inferiormente. Suponha que J é limitada inferiormente e defina

$$
c=\inf \{J(u) ; u \in E\} \text {. }
$$

Então, para todo $\epsilon>0$, existe $u_{\epsilon} \in E$ tal que

$$
c \leq J\left(u_{\epsilon}\right) \leq c+\epsilon
$$

e para todo $u \in E$ tal que $u \neq u_{\epsilon}$, temos

$$
J(u)-J\left(u_{\epsilon}\right)+\epsilon d\left(u, u_{\epsilon}\right)>0 .
$$

REFERÊNCIA. Ekeland [46].

Teorema A.8 (Princípio Variacional de Ekeland, 2 versão) Sejam E um espaço de Banach e $G \in C^{2}(E, \mathbb{R})$ tal que $G^{\prime}(v) \neq 0$, para todo $v \in V=\{v \in E \mid G(v)=1\}$. Seja $F \in C^{1}(E, \mathbb{R})$ limitado inferiormente em $V, v \in V$ e $\epsilon, \delta>0$. Se

$$
F(v) \leq \inf _{V} F+\epsilon
$$

então existe $u \in V$ tal que

$$
F(u) \leq \inf _{V} F+2 \epsilon, \min _{\lambda \in \mathbb{R}}\left\|F^{\prime}(u)-\lambda G^{\prime}(u)\right\| \leq \frac{8 \epsilon}{\delta} \quad e\|u-v\| \leq 2 \delta
$$


REFERÊNCIA. Willem [81], pág. 122.

\section{A.7 Princípio da Criticalidade Simétrica de Palais}

Teorema A.9 (Princípio da Criticalidade Simétrica de Palais) Sejam H um espaço de Hilbert e $G$ um grupo topológico. Suponha que uma ação de $G$ sobre o espaço $H$ seja isométrica e suponha que $I \in C^{1}(H, \mathbb{R})$ é invariante pela ação. Suponha que u é um ponto crítico de I restrito ao Fix $(G)$, que é definido por

$$
\operatorname{Fix}(G):=\{u \in H ; g u=u, \forall g \in G\}
$$

onde $g u(x)=u\left(g^{-1} x\right), \forall x \in H$. Então, u é ponto crítico de I em $H$.

ReFERÊnCIA. Palais [61].

Uma versão do Princípio da Criticalidade Simétrica de Palais para espaços de Banach reflexivos pode ser encontrada em [59].

\section{A.8 Gênero}

Seja $E$ um espaço de Banach real e defina

$$
\Sigma:=\{Y \subset E \backslash\{0\} ; Y \text { é fechado em } E \text { e } Y=-Y\}
$$

Dizemos que o gênero de $Y$ é igual a $n$ e denotamos por $\gamma(Y)=n$, se existe uma aplicação ímpar $\phi \in C\left(Y, \mathbb{R}^{n} \backslash\{0\}\right)$ e $n$ é o menor inteiro com esta propriedade. Quando não existe tal número finito $n$, consideramos $\gamma(Y)=\infty$. Para $Y=\emptyset$, temos $\gamma(\emptyset)=0$. A seguir, listamos algumas propriedades envolvendo o gênero.

Proposição A.1 Sejam $A, B \in \Sigma$. Então, 
(i) Se $\gamma(A)>1$, então A contém uma quantidade infinita de pontos distintos.

(ii) Se existe uma aplicação ímpar $h \in C(A, B)$, então $\gamma(A) \leq \gamma(B)$.

(iii) Se $A \subset B$, então $\gamma(A) \leq \gamma(B)$.

(iv) $\gamma(A \cup B) \leq \gamma(A)+\gamma(B)$.

(v) Se $X$ é um subespaço de $E$ de codimensão $k$ e $\gamma(A)>k$, então $A \cap X \neq \emptyset$.

(vi) Se A é compacto, então $\gamma(A)<\infty$ e existe um $\delta>0$ tal que $N_{\delta}(A) \in \Sigma e$ $\gamma\left(N_{\delta}(A)\right)=\gamma(A)$, onde $N_{\delta}(A)=\left\{u \in E ;\|u-a\|_{E} \leq \delta, \forall a \in A\right\}$.

(vii) Se $\gamma(B)<\infty$, então $\gamma(\overline{A \backslash B}) \geq \gamma(A)-\gamma(B)$.

(viii) Se $\Theta$ é uma vizinhança limitada de 0 em $\mathbb{R}^{k}$ e existe um homeomorfismo impar $h \in C(A, \partial \Theta)$, então $\gamma(A)=k$.

REFERÊnCIA. Rabinowitz [66], pág. 46.

\section{A.9 Categoria}

As seguintes definições, notações e resultados envolvendo Categoria de LusternikSchnirelman podem ser encontrados em [81, Capítulo 5.

Definição A.2 Um subconjunto fechado A é contrátil em um espaço topológico $X$ se existe $h \in C([0,1] \times A, X))$ tal que

$$
h(0, u)=u \quad e \quad h(1, u)=h(1, v), \quad \forall u, v \in A .
$$

Definição A.3 Sejam A, B, Y subconjuntos fechados de um espaço topológico $X$. Então, por definição, $A \prec_{Y} B$ em $X$ se $Y \subset A \cap B$ e existe $\left.h \in C([0,1] \times A, X)\right)$ tal que 
a) $h(0, u)=u, h(1, u) \in B, \forall u \in A$;

b) $h(t, Y) \subset Y, \forall t \in[0,1]$.

Definição A.4 Sejam $Y$ e A subconjuntos fechados de um espaço topológico $X$, com $Y \subset A$. A categoria de $A$ em $X$ relativa a $Y$, denotada por cat ${ }_{X, Y}(A)$, é o menor inteiro $n$ tal que existe $n+1$ subconjuntos fechados $A_{0}, A_{1}, \ldots, A_{n}$ de $X$ safisfazendo

a) $A=\cup_{j=0}^{n} A_{j}$;

b) $A_{1}, \ldots, A_{n}$ são contráteis em $X$;

c) $A_{0} \prec_{Y} Y$ em $X$.

$A$ categoria de $A$ em $X$ é definida por $\operatorname{cat}_{X}(A):=\operatorname{cat}_{X, \emptyset}(A)$.

Lema A.7 Sejam A,B,C,Y tais que $Y \subset A . A$ categoria relativa satisfaz as seguintes propriedades:

(i) Se $Y \subset B \cap C, A \prec_{Y} B$ e $B \prec_{Y} C$ em $X$, então $A \prec_{Y} C$ em $X$.

(ii) $\operatorname{cat}_{X, Y}(Y)=0$.

(iii) $\operatorname{cat}_{X, Y}(A \cup B) \leq \operatorname{cat}_{X, Y}(A)+\operatorname{cat}_{X}(B)$.

(iv) Se $A \prec_{Y} B$, então $\operatorname{cat}_{X, Y}(A) \leq \operatorname{cat}_{X, Y}(B)$.

Teorema A.10 Se I $\left.\right|_{V}$ é limitado inferiormente e satisfaz a condição $(P . S)_{c}$, para qualquer $c \in\left[\inf _{V} I, d\right]$, então $\left.I\right|_{V}$ possui um mínimo e $I^{d}$ contém pelo menos cat ${ }_{I^{d}}\left(I^{d}\right)$ de pontos críticos de $\left.I\right|_{V}$. 


\section{REFERÊNCIAS BIBLIOGRÁFICAS}

[1] Adams R. A. Sobolev Spaces, Academic Press Inc., New York (1975).

[2] Adimurthi Existence of positive solutions of the semilinear Dirichlet problems with critical growth for the N-Laplacian, Ann. Scuola Norm. Sup. Pisa 17 (1990), 393-413.

[3] Adimurthi \& Yadava S. L. Multiplicity results for semilinear equations in bounded domain of $\mathbb{R}^{2}$ involving critical exponent, Ann. Scuola Norm. Sup. Pisa Cl. Sci. 17 (1990), 481-504.

[4] Alama S. \& Tarantello G. On semilinear elliptic equations with indefinite nonlinearities, Calc of Var. and PDE 1 (1993), 439-475.

[5] Alama S. \& Tarantello G. Elliptic problems with nonlinearities indefinite in sign, J. Func. Anal. 141 (1996), 159-215.

[6] Alves C. O. Multiplicity of solutions for a class of elliptic problem in $\mathbb{R}^{2}$ with Neumann conditions, J. Diff. Eq. 219 (2005), 20-39.

[7] Alves C. O. Existence and multiplicity of solutions for a class of quasilinear equation, Adv. Nonlinear Studies 5 (2005), 73-87.

[8] Alves C. O. Multiple positive solutions for equations involving critical Sobolev exponent in $\mathbb{R}^{N}$, Elect. J. Diff. Eq. 13 (1997), 1-10.

[9] Alves C. O., Bezerra do Ó J. M. \& Miyagaki O. H. On pertubations of a class of periodic m-Laplacian equation with critical growth, Nonlinear Analysis-TMA 45 (2001), 849-863. 
[10] Alves C. O., Bezerra do Ó J. M. \& Miyagaki O. H. On nonlinear pertubations of a periodic elliptic problem in $\mathbb{R}^{2}$ involving critical growth, Nonlinear Anal. 56 (2004), 781-791.

[11] Alves C. O. \& Ding Y. H. Multiplicity of positive solutions to a p-Laplacian equation involving critical nonlinearity, J. Math Anal. Appl. 279 (2003), 508-521.

[12] Alves C. O.\& Figueiredo G. M. On multiplicity and concentration of positive solutions for a class of quasilinear problems with critical exponential growth in $\mathbb{R}^{N}$, J.D.E. 246 (2009), 1288-1311.

[13] Ambrosetti A., Brezis H. \& Cerami G. Combined effects of concave and convex nonlinearities in some elliptic problems, J. Funct. Anal. 122 (1994), 519-543.

[14] Ambrosetti A. \& Rabinowitz P. H. Dual variational methods in critical point theory and applications, J. Funct. Anal. 14 (1973), 349-381.

[15] Aris R. The Mathematical theory of Diffusion and Reaction in Permeable Catalyst, Vol. I, II., Clarendon Press, Oxford (1975).

[16] Azorero J. G. \& Peral I. Existence and nonuniqueness for a p-Laplacian: Nonlinear eigenvalues, Comm. Partial Diferential Equations 12 (1987), 1389-1430.

[17] Azorero J. G. \& Peral I. Multiplicity of solutions for elliptic problems with critical exponent or with a nonsymetric term, Amer. Math. Soc. 2 (1991), 877-895.

[18] Bahri A. \& Coron J.M. On a nonlinear elliptic equation involving the critical Sobolev exponent: The effect of the topology of the domain, Comm. Pure Appl. Math. 41 (1988), 253-294.

[19] Benci V. \& Cerami G. The effect of the domain topology on the number of positive solutions of nonlinear elliptic problems, Arch. Rat. Mech. Anal. 114 (1991), 79-93.

[20] Benci V. \& Cerami G. Positive solutions of some nonlinear elliptic problems in exterior domains, Arch. Rational Mech. Anal. 99 (1987), 283-300.

[21] Benci V. \& Cerami G. Multiple positive solutions of some elliptic problems via the Morse theory and the domain topology, Cal. Var. 02 (1994), 29-48. 
[22] Berestycki H. , Capuzzo-Dolcetta I. \& Nirenberg L. Variational methods for indefinite superlinear homogeneous elliptic problems, NoDEA Nonlinear Differential Equations Appl. (1995), 553-572.

[23] Berestycki H. , Capuzzo-Dolcetta I. \& Nirenberg L. Superlinear indefinide elliptic problems and nonlinear liouville theorems, Topol. Meth. Nonl. Anal. 4 (1994), 59-78.

[24] Berestycki H. \& Lions P. L. Nonlinear scalar field equations, I. Existence of ground state, Arch. Ration. Mech. Anal. 82 (1983), 313-345

[25] Bernis F., Azorero J. G. \& Peral I. Existence and Multiplicity of nontrivial solutions in semilinear critical problems of fourth order, Adv. Diff. Eq. 1 (1996), 219-240.

[26] Bezerra do Ó J.M.B. N-Laplacian equations in $\mathbb{R}^{N}$ with critical growth, Abstr. Appl. Anal. 2 (1997), 301-315.

[27] Bezerra do Ó J. M. B. Semilinear Dirichlet problems for the N-Laplacian in $\mathbb{R}^{N}$ with nonlinearities in critical growth range, Differential Integral Equations 5 (1996), 967-979.

[28] Bezerra do Ó J. M. B., Medeiros E. \& Severo U. A nonhomogeneos elliptic problem involving critical growth in dimension two, J. Math. Anal. Appl. 345 (2008), 286-304.

[29] Bezerra do Ó J. M. B., Medeiros E. \& Severo U. On a quasilinear nonhomogeneos elliptic equation with critical growth in $\mathbb{R}^{N}$, J. Diff. Equations 246 (2009), 1363-1386.

[30] Bognar G. Numerical and Numerical and Analytic Investigation of Some Nonlinear Problems in Fluid Mechanics, Computer and Simulation in Modern Science, Vol.II, WSEAS Press (2008), 172-179.

[31] Bonder J. F. \& Rossi J. D. A fourth order elliptic equation with nonlinear boundary conditions, Nonlinear Analysis 49 (2002), 1037-1047.

[32] Brezis H. Análisis funcional. Teoría y aplicaciones, Alianza Editorial S. A., Madrid (1984).

[33] Brezis H. \& Nirenberg L. Positive solutions of nonlinear elliptic equations involving critical Sobolev exponents, Comm. Pure Appl. Math 36 (1983), 437-477.

[34] Byeon J. Existence of many nonequivalent nonradial positive solutions of semilinear elliptic equations on three-dimensional annuli, J. of Diff. Eq. 136 (1997), 136-165. 
[35] Cao D. M. Nontrivial solution of semilinear elliptic equation with critical exponent in $\mathbb{R}^{2}$, Commun. Partial Differential Equations 17 (1992), 407-435.

[36] Cao D. M., Li G.-B. \& Zhou H.-S. Multiple solutions for Non-homogeneous Elliptic Equations with Critical Sobolev Exponent, Proc. of the Royal Soc. of Edinburgh 124A (1994), 1177-1191.

[37] Castro A. \& Finan B.M. Existence of many positive nonradial solutions for a superlinear Dirichlet problem on thin annuli, Nonlinear Diff. Equations 5 (2000), 21-31.

[38] Catrina F. \& Wang Z.-Q. Nonlinear elliptic equations on expanding symmetric domains, J. of Diff. Equations 156 (1999), 153-181.

[39] Cerami G. \& Passaseo D. Existence and multiplicity of positive solutions for nonlinear elliptic problems in exterior domains with rich topology, J. Nonlinear Anal. T.M.A. 18 (1992), 103-119.

[40] Coffman C. A non-linear boundary value problem with many positive solutions, J. Diff. Equa. 54 (1984), 429-437.

[41] Costa D.G. \& Tehrani H. T. Existence of positive solutions for a class of indefinite elliptic problems in $\mathbb{R}^{N}$, Calc. Var.13 (2001), 159-189.

[42] de Figueiredo D. G., Gossez J. P. \& Ubilla P. Local superlinearity and sublinearity for indefinite semilinear elliptic problems, Journal of Functional Analysis 199 (2003), 452467.

[43] de Figueiredo D. G. \& Miyagaki O. H. Multiplicity of non-radial solutions of critical elliptic problems in an annulus, Proc. R. Soc. Edinburgh 135A (2005), 25-37.

[44] de Figueiredo D. G., Miyagaki O. H. \& Ruf B. Elliptic equations in $\mathbb{R}^{2}$ with nonlinearities in the critical growth range, Calc. Var. Partial Differential Equations 3 (1995), 139-153.

[45] Egnell H. Existence and nonexistence results for $m$-Laplace equations involving critical Sobolev exponents, Arch. Ration. Mech. Analysis 104 (1988), 57-77.

[46] Ekeland I. On the variational principle, J. Math. Anal. Appl. 47 (1974), 324-353.

[47] Evans L. C. Partial differential equations, Graduate Studies in Mathematics, American Mathematical Society, Providence, Rhode Island, vol. 19 (1998). 
[48] Guedda M. \& Veron L. Quasilinear elliptic equations involving Sobolev exponents, Nonlinear Analysis 13 (1989), 879-902.

[49] Gidas B., Ni W. N. \& Nirenberg L. Symmetric and related proprieties via the maximum principle, Commun. Math. Phys. 68 (1979), 209-243.

[50] Glowinski R. \& Rappaz J. Approximation of nonlinear elliptic problem arising in a non-Newtonian fluid flow model in glaciology, Math. Model. Numer. Anal. 37 (2003), 175-186.

[51] Gonçalves J. V. \& Alves C. O. Existence of positive solutions for a m-laplacian equations in $\mathbb{R}^{N}$ involving critical Sobolev exponents, Nonlinear Anal. TMA 32 (1998), 53-70.

[52] Hirano N. \& Mizoguchi N. Nonradial solutions of semilinear elliptic equations on annuli, J. Math. Soc. Japan 46 (1994), 111-117.

[53] Kavian O. Introduction à la théorie des points critiques et applications aux problèms elliptiques, Springer-Verlag (1993).

[54] Li. Y. Y. Existence of many positive solutions of semilinear elliptic equations on annulus, J. Diff. Eqns 83 (1990), 348-367.

[55] Lin S. S. Existence of many positive nonradial solutions for nonlinear elliptic equations on an annulus, J. Diff. Equa. 103 (1993), 338-349.

[56] Mastorakis N. E. \& Fathabadi H. On the solution of p-Laplacian for non-Newtonian fluid flow, Wseas Trans. on Math. 8 (2009), 238-245.

[57] Mizoguchi N \& Suzuki T. Semilinear elliptic equations on a annuli in three and higher dimensions, Houston J. of Math. 22 (1996), 199-215.

[58] Morais Filho D. C. \& Miyagaki O. H. Critical singular problems on unbounded domains, Abstr. Appl. Anal. 6 (2005), 639-653.

[59] Morais Filho D. C., Souto M. A. S. \& Bezerra do Ó J. M. A compactness embedding lemma, a principle of symmetric criticality and applications to elliptic problems, Universidad Católica del Norte 19 (2000), 1-17.

[60] Moser J. A sharp form of an inequality by N. Trudinger, Indiana Univ. Math. J. 20 (1971), 1077-1092. 
[61] Palais R. S. The principle of symmetric criticality, Commun. Math. Phys. 69 (1979), 19-30.

[62] Pan X. Positive solutions of the elliptic equations $\Delta u+u^{\frac{N+2}{N-2}}+K(x) u^{q}=0$ in $\mathbb{R}^{N}$ and balls, J. Math. Anal. Appl. 172 (1993) 323-338.

[63] Panda R. On semilinear Neumann problems with critical growth for the N-Laplacian, Nonlinear Anal 26 (1996), 1347-1366.

[64] Pelissier M. C. \& Reynaud L. Étude d'un modèle mathématique d'ecoulement de glacier, C. R. Acad. Sci., Paris, Sér. A 279 (1974), 531-534. (French)

[65] Peral I. Multiplicity of solutions for the p-Laplacian, Second School on Nonlinear Functional Analysis e Appl. Diff. Eqns., I.C.T.P.I., Trieste (1997).

[66] Rabinowitz P. H. Minimax methods in critical point theory with applications to differential equations, CBMS Reg. Conf. series in math. 65 (1984).

[67] Rey O. A multiplicity result for a variational problem with lack of compactness, J. Nonlinear Anal. T.M.A. 13 (1989), 1241-1249.

[68] Royden H. L. Real Analysis, 2 ed. The Macmillan Company (1988).

[69] Schoenauer M. A monodimensional model for fracturing, In A. Fasano and M. Primicerio (editors): Free Boundary Problems, Theory Applications, Pitman Research Notes in Mathematics 79, Vol. II., London, 701-711 (1983).

[70] Silva E. A. B. \& Soares S. H. M. Liouville-Gelfand type problems for the N-Laplacian on bounded domains of $\mathbb{R}^{N}$, Ann. Scuola Norm. Sup. Pisa Cl. Sci. 4 (1999), 1-30.

[71] Simon J. Regularité de la solution d'une equation non linéaire dans $R^{n}$, Lectures Notes in Math. 665, Springer Verlag, Berlin (1978).

[72] Suzuki T. Positive solutions for semilinear elliptic equations on expanding annului: Mountain Pass approach, Funkcialaj Ekvacioj 39 (1996) 143-164.

[73] Tarantello G. On nonhomogeneous elliptic equations involving critical sobolev exponent, Ann. Inst. H. Poincaré Anal. Non Lineáire 9 (1992), 243-261.

[74] Tehrani H. T. Solutions for indefinite semilinear elliptic equations in exterior domains, J. Math. Anal. Appl. 255 (2001), 308-318. 
[75] Tehrani H.T. Infinitely many solutions for indefinite semilinear elliptic equations without symmetry, Comm. P.D.E. 21 (1996), 541-557.

[76] Tonkes, E. Solutions to a pertubed critical semilinear equation concerning the $N$ Laplacian in $\mathbb{R}^{N}$, Comment. Math. Univ. Carolinae 40 (1999), 679-699.

[77] Trudinger N. On imbedding into Orlicz space and some applications, J. Math. Mech. 17 (1967), 473-484.

[78] Trudinger N. On Harnack type inequalities and their applications to quasilinear elliptic equations, Comm. Pure Appl. Math. (1967), 721-747.

[79] Wang Z. \& Willem M. Existence of many positive solutions of semilinear elliptic equations on an annulus, Proc. Amer. Math. Soc. 127 (1999), 1711-1714.

[80] Wang Y., Yang J. \& Zhang Y. Quasilinear elliptic equations involving the $N$-Laplacian with critical exponential growth in $\mathbb{R}^{N}$, Nonlinear Analysis 71 (2009), 6157-6169.

[81] Willem M. Minimax Theorems, Birkhauser (1986).

[82] Xavier M. S. Problemas elípticos quasilineares com crescimento crítico na presença de simetria, Tese de doutorado (2004).

[83] Zhihui W. \& Xinmin W. A multiplicity result for quasilinear ellipitic equations involving critical Sobolev exponents, Nonlinear Anal. TMA 18 (1992), 559-567. 